\title{
Four dimensional manifolds constructed by lens space surgeries along torus knots
}

\author{
Motoo TANGE and Yuichi YAMADA
}

June 18, 2012

\begin{abstract}
A framed knot with an integral coefficient determines a simply-connected 4-manifold by 2-handle attachment. Its boundary is a 3-manifold obtained by Dehn surgery along the framed knot. For a pair of such Dehn surgeries along distinct knots whose results are homeomorphic, it is a natural problem: Determine the closed 4-manifold obtained by pasting the 4-manifolds along their boundaries. We determine the complete list (set) of pairs of integral surgeries along distinct torus knots whose resulting manifolds are orientation preserving/reversing homeomorphic lens spaces, and study the closed 4-manifolds constructed as above. The list consists of five sequences. All framed links and Kirby calculus are indexed by integers. As a by-product, some sequences of embeddings of lens spaces into the standard 4-manifolds are constructed.
\end{abstract}

\section{Contents}

1 Introduction $\mathbf{2}$

1.1 First sequence: Lens spaces with same orientations . . . . . . . . . . . . 5

1.2 Second sequences: Lens spaces with opposite orientations I . . . . . . . . . . 6

1.3 Third sequences: Lens spaces with opposite orientations II . . . . . . . . . . . 7

2 Preliminaries $\quad 10$

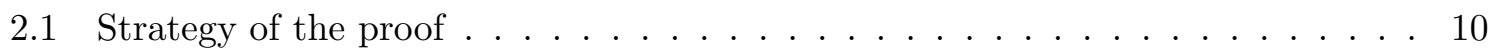

2.2 Notations . . . . . . . . . . . . . . . . . . . . . . 11

2.3 Framed chain links, Lens spaces and Torus knots . . . . . . . . . . . . . 13

2.4 Contribution of pasting maps . . . . . . . . . . . . . . . 17

3 Formulas on the sequences $\quad 17$

3.1 Formulas on the first sequence . . . . . . . . . . . . . . . 18

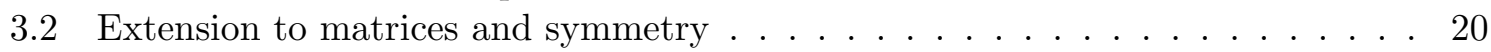

3.3 Trivial cases . . . . . . . . . . . . . . . . . . . . . 22

\footnotetext{
${ }^{0} 2000$ Mathematics Subject Classification: Primary 57M25, 57N35, Secondary 57M99.

Keywords: 4-manifold, Dehn surgery, handle calculus.

${ }^{1}$ The first author is partially supported by JSPS Research Fellowships for Young Scientists (21-1458). The second author was supported by KAKENHI (Grant-in-Aid for Scientific Research) No.21540072 and No.18740029.
} 


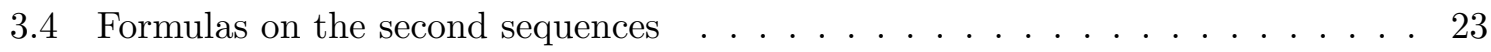

3.5 Formulas on the third sequences $\ldots \ldots \ldots \ldots \ldots \ldots$

3.6 Proof of the properties (4) in Lemmas . . . . . . . . . . . . 28

4 3-dim calculus $\quad 31$

4.1 For the first sequence . . . . . . . . . . . . . . . . . 31

4.2 For the second and the third sequences . . . . . . . . . . . . 34

5 4-dim calculus $\quad 38$

5.1 Key lemma: Handle-slides in a thickened torus . . . . . . . . . . . . . . 38

5.2 4-dim calculus for each sequence . . . . . . . . . . . . . . . 39

5.3 Demonstration in the case $i=2 \ldots \ldots \ldots \ldots \ldots \ldots$. . . . . . . . . . 41

6 Completeness of the list $\quad 42$

6.1 First case: Lens spaces with same orientations . . . . . . . . . . . . . . . . 43

6.2 Second case: Lens spaces with opposite orientations I . . . . . . . . . . . . . 46

6.3 Third case: Lens spaces with opposite orientations II . . . . . . . . . . . . . . 52

\section{Introduction}

A framed link $(L ; \boldsymbol{n})=\left(K_{1} \cup K_{2} \cup \cdots \cup K_{r} ; n_{1}, n_{2}, \ldots, n_{r}\right)$ in the 3-sphere $S^{3}\left(=\partial B^{4}\right)$ is a disjoint union of framed knots $\left(K_{i} ; n_{i}\right)$, where $K_{i}$ is a component of the link $L$ and $n_{i}$ is an integer, called a framing or a coefficient. By $X(L ; \boldsymbol{n})$, we denote a simply-connected 4-manifold obtained by attaching 2-handles to a 4-ball $B^{4}$ (a 0-handle) along the knots $K_{i}$ with $n_{i}$-framing. Its boundary $\partial X(L ; \boldsymbol{n})$ is a 3 -manifold $M(L ; \boldsymbol{n})$ obtained by Dehn surgery along the framed link $(L ; \boldsymbol{n})$. As a Dehn surgery description of 3-manifolds, the coefficient $n_{i}$ can be a rational number or $1 / 0(=\infty)$, see Rolfsen $[\mathrm{R}]$. They are visualized by the usual link diagrams with labeling. They are called Kirby diagrams. The resulting manifold does not depend on the orientation of the components. In this paper, when we regard a diagram as a description of a 4-manifold, we call it a 4-dim diagram, when we regard the diagram as a Dehn surgery description of a 3-manifold, we call it a 3-dim diagram. A method Kirby calculus is known to prove a diffeomorphism between any pair of 4-manifolds or 3-manifolds described by Kirby diagrams. Depending on which dimensional manifolds are described, we call a calculus 4-dim calculus or 3-dim calculus. For Kirby diagrams and Kirby calculus, see $[\mathrm{K}, \mathrm{K} 2, \mathrm{GS}]$.

Suppose that two oriented 4-manifolds $X_{1}$ and $X_{2}$ have homeomorphic boundaries with the same or the opposite orientations. If a homeomorphism $\varphi$ between the boundaries is an orientation-preserving (or an orientation-reversing, respectively), we choose $-X_{2}$ (or $X_{2}$ ), where $\mp X_{2}$ means $X_{2}$ with the opposite (for - ) or the same (for + ) orientation. We can construct an oriented 4-manifold $X_{1} \cup_{\varphi}\left(\mp X_{2}\right)$, where $\cup_{\varphi}$ means pasting $X_{1}$ and $\mp X_{2}$ along the boundaries by the orientation-reversing homeomorphism $\varphi$. It is known that any homeomorphism over a closed 3-manifold is approximated by a diffeomorphism. Thus we can assume that the constructed 4-manifold has a differential structure. It is a natural problem:

Problem 1.1 Is the 4-manifold $X_{1} \cup_{\varphi}\left(\mp X_{2}\right)$ a standard 4-manifold, for any homeomorphism $\varphi$ ? 
This can be extended to

Problem 1.2 For a 4-manifold $X$ that contains a copy of $X_{1}$, is $X^{\prime}=\left(X \backslash\right.$ int $\left.X_{1}\right) \cup_{\partial}\left( \pm X_{2}\right)$ (always) homeomorphic or diffeomorphic to the initial manifold $X$ ?

To define such an operation using simpler pair $X_{1}, X_{2}$ is one of our motivations.

We are concerned with the case where the common boundary is a lens space. Dehn surgeries along knots (with integer coefficients) whose results are lens spaces are called lens space surgery. Our purposes are

(I) (There are some overlap with Saito-Teragaito [ST]) Study pairs $(K ; n),\left(K^{\prime} ; n^{\prime}\right)$ of lens space surgeries along distinct torus knots with integer coefficients whose results are homeomorphic lens spaces $M(K ; n) \cong \pm M\left(K^{\prime} ; n^{\prime}\right)$, and

(II) Study Problem 1.1 on $X(K ; n) \cup_{\varphi}\left(\mp X\left(K^{\prime} ; n^{\prime}\right)\right)$ for each pair in (I).

Note that $M(K ; n) \cong \pm M\left(K^{\prime} ; n^{\prime}\right)$ implies $n^{\prime}= \pm n$ by the first homology. Since $M(K ! ;-n) \cong$ $-M(K ; n)$ holds in general, we assume $n^{\prime}=n$, where $K$ ! means a mirror image of $K$.

In [Mo], Moser studied Dehn surgery along torus knots and proved that $(A B \pm 1)$-surgery along a torus knot $T(A, B)$ is a lens space:

Lemma 1.3 (Moser's lens space surgery $[\mathrm{Mo}]$ )

$$
M(T(A, B) ; A B \pm 1)=-L\left(A B \pm 1, A^{2}\right) .
$$

Our convention about orientations of lens spaces is " $p / q$ Dehn surgery along an unknot is $-L(p, q)$ ". Note that $L\left(A B \pm 1, A^{2}\right) \cong L\left(A B \pm 1, B^{2}\right)$, since $A^{2} B^{2} \equiv 1 \bmod (A B \pm 1)$, see Subsection 2.3. Here, we choose the same sign $+($ or -$)$ in the double signs $( \pm)$. It is also proved that lens space surgeries along (non-trivial) torus knots with integer coefficients are restricted to the surgeries above.

Torus knots have some symmetries: $T(B, A)$ is equivalent to $T(A, B), T(-A,-B)$ is equivalent to $T(A, B)$, and $T(A,-B)=T(A, B)$ !. Since $M(K ! ;-n) \cong-M(K ; n)$, we treat with only positive torus knots, i.e., $T(A, B)$ with $A>0$ and $B>0$. For a torus knot $K=T(A, B)$, we call $(A, B)$ type of $K$, identifying $(B, A)$ and $(A, B)$.

Here we make a survey of our results: In the next three subsections, we will define five sequences of pairs of pairs of coprime positive (with a few exception) integers:

(1) $[A B C D]=((A, B),(C, D))$, indexed by two integers $a$ and $i$.

(2) $[S T U V]=((S, T),(U, V))$ and $[s t u v]=((s, t),(u, v))$, both indexed by integers $i$.

(3) $[K L M N]=((K, L),(M, N))$ and $[k l m n]=((k, l),(m, n))$, both indexed by integers $i$.

We will use the notations [..] as a name of the sequence. The sequence (1) corresponds to lens space surgeries along positive torus knots whose lens spaces (the result of the Dehn surgery) are orientation-preservingly homeomorphic. The smallest nontrivial example is 21surgery along $T(4,5)$ and that along $T(11,2)$, whose resulting lens space is $-L(21,4)$. The sequences (2) and (3) correspond to lens space surgeries along positive torus knots whose lens spaces are orientation-reversingly homeomorphic. The smallest nontrivial example in (2) is 
13-surgery along $T(4,3)$ and that along $T(2,7)$, whose resulting lens space is $\pm L(13,4)$. For more concrete examples, see Tables at the end of this section. We call the set of the pairs of pairs in the sequences in (1), (2) and (3) a list (of lens space surgeries along positive torus knots whose lens spaces are homeomorphic).

Our results are summarized as follows:

Theorem 1.4 (Completeness of the list) The list is complete in the following sense: If $\left(K, K^{\prime}\right)$ is a pair of distinct positive torus knots satisfying that $M(K ; n) \cong \pm M\left(K^{\prime} ; n\right)$ and it is a lens space, then the types of $\left(K, K^{\prime}\right)$ or that of $\left(K^{\prime}, K\right)$ is included in the list.

Theorem 1.5 (4-dim problem) For every pair of lens space surgeries along positive torus knots whose resulting lens spaces are homeomorphic, the corresponding 4-manifold in the sense of Problem 1.1 is a standard 4-manifold, i.e., diffeomorphic to one of $S^{2} \times S^{2}, S^{2} \tilde{\times} S^{2}$ $\left(\cong \mathbb{C} P^{2} \sharp \overline{\mathbb{C} P^{2}}\right)$ or $\mathbb{C} P^{2} \sharp \mathbb{C} P^{2}$.

Remark 1.6 For Problem 1.1, we have to study contribution of pasting maps between the boundaries of the pieces. We will argue about it in Subsection 2.4. The diffeotopy groups $\pi_{0}\left(\operatorname{Diff}^{+}(L(p, q))\right)$ of lens spaces $L(p, q)$ are studied by Bonahon [Bon] and HodgsonRubinstein [HR].

Remark 1.7 Saito-Teragaito [ST] have considered many families of pairs of lens space surgery yielding homeomorphic lens spaces, along not only torus knots but also non-torus (i.e., cable and hyperbolic) knots, mainly from the view point of existence of such pairs. They only studied (wrote) the sequence $[S T U V]$, but did not refer to the other sequences $[A B C D],[s t u v],[K L M N]$ and $[k l m n]$.

Remark 1.8 By Donaldson's and Freedman's theorems, the homeomorphisms (instead of diffeomorphism) in Theorem 1.5 can be shown by 1-connectedness and intersection form of the constructed 4-manifold (Any closed 1-connected smooth 4-manifold whose intersection form is rank two and even is homeomorphic to $S^{2} \times S^{2}$. Any closed positive definite 1-connected smooth 4-manifold is known to be homeomorphic to a connected sum of some copies of $\mathbb{C} P^{2}$ ), see Remark 1 in Ue [U]. We study whether the constructed 4-manifolds are diffeomorphic to the standard one.

Remark 1.9 As a by-product of Theorem 1.5, we have some families of smooth embeddings of lens spaces into the standard 4-manifolds, see Corollary 1.19. In Sasahira [Sa], a pair of lens spaces $L(P, Q)$ and $L\left(P, Q^{\prime}\right)$ (with the same $P$ ) that satisfies the following condition is constructed: One can not be smoothly embedded into $\mathbb{C} P^{2} \sharp \mathbb{C} P^{2}$ but the other can. Embedding of $L\left(P, Q^{\prime}\right)$ is a motivation for the authors to update the present paper. Here the authors thank to Prof. Sasahira [Sa] for his interest in our examples and for communication on lens spaces embedded in 4-manifolds.

Now we introduce the definitions (constructions) and details on the sequences. They are divided into three subsections. 


\subsection{First sequence: Lens spaces with same orientations}

We define a sequence of pairs that give lens space surgeries along positive torus knots whose lens spaces are orientation-preservingly homeomorphic.

Definition $1.10 \quad([A B C D])$

Let $a, i$ be integers with $i \geq 0$. We often fix $a$. We define a sequence of pairs $(A(a, i), B(a, i))$ and $(C(a, i), D(a, i))$ inductively with respect to $i$ as below. They will be denoted by $\left(A_{i}, B_{i}\right)$ and $\left(C_{i}, D_{i}\right)$ for short, respectively.

$$
\left(\begin{array}{l}
A_{0} \\
B_{0}
\end{array}\right)=\left(\begin{array}{c}
-1 \\
1
\end{array}\right), \quad\left(\begin{array}{c}
C_{0} \\
D_{0}
\end{array}\right)=\left(\begin{array}{l}
1 \\
1
\end{array}\right)
$$

and

$$
\left(\begin{array}{l}
A_{i+1} \\
B_{i+1}
\end{array}\right)=\left(\begin{array}{cc}
1 & 0 \\
a-2 & 1
\end{array}\right)\left(\begin{array}{l}
C_{i} \\
D_{i}
\end{array}\right), \quad\left(\begin{array}{l}
C_{i+1} \\
D_{i+1}
\end{array}\right)=\left(\begin{array}{cc}
1 & a+2 \\
0 & 1
\end{array}\right)\left(\begin{array}{l}
A_{i} \\
B_{i}
\end{array}\right) .
$$

We often assume $a \geq 3$ and $i \geq 2$ for nontriviality (see Subsection 3.3). In the next section, we will show:

Lemma 1.11 The pairs $\left(A_{i}, B_{i}\right)$ and $\left(C_{i}, D_{i}\right)$ satisfy the followings:

(1) Each pair $\left(A_{i}, B_{i}\right)$ and $\left(C_{i}, D_{i}\right)$ is coprime.

(2) $A_{i} B_{i}+1=C_{i} D_{i}-1$. We call this integer $P_{i}$.

(3) $B_{i}^{2} \equiv D_{i}^{2} \bmod P_{i}$

(4) If $a \geq 3$ and $i \geq 2$, then $A_{i}^{4} \not \equiv \pm 1 \bmod P_{i}$.

By (1)-(3) in this lemma and Moser's results, we have a sequence of pairs of lens space surgeries along distinct torus knots whose lens spaces are orientation-preservingly homeomorphic:

$$
M\left(T\left(A_{i}, B_{i}\right) ; A_{i} B_{i}+1\right) \cong M\left(T\left(C_{i}, D_{i}\right) ; C_{i} D_{i}-1\right) \cong-L\left(P_{i}, B_{i}{ }^{2}\right) .
$$

In other words, $-L\left(P_{i}, B_{i}{ }^{2}\right)$ bounds each 4 -manifold below:

$$
\begin{aligned}
& X_{A B}(a, i):=X\left(T\left(A_{i}, B_{i}\right) ; A_{i} B_{i}+1\right), \\
& X_{C D}(a, i):=X\left(T\left(C_{i}, D_{i}\right) ; C_{i} D_{i}-1\right) .
\end{aligned}
$$

We often call them $X_{A B}, X_{C D}$ for short, respectively.

Boyer [Boy] proved that $X_{A B}$ is not homeomorphic to $X_{C D}$ by consideration on the homology long exact sequence, intersection forms and mapping class groups of the lens spaces. The main theorem on the first sequence is:

Theorem 1.12 We assume $a \geq 3$ and $i \geq 2$. Let $X_{A B}=X_{A B}(a, i)$ and $X_{C D}=X_{C D}(a, i)$ be the 4-manifolds as above. Then the smooth 4-manifold $X_{A B} \cup\left(-X_{C D}\right)$ obtained by pasting $X_{A B}$ and $-X_{C D}$ by any orientation-reversing homeomorphism between their boundaries is diffeomorphic to $S^{2} \times S^{2}$, or $S^{2} \tilde{\times} S^{2}$ according to whether a is even or odd (independent of $i$ ):

$$
X_{A B} \cup\left(-X_{C D}\right) \cong\left\{\begin{array}{ll}
S^{2} \times S^{2} & \text { if a is even } \\
S^{2} \tilde{\times} S^{2} & \text { if a is odd }
\end{array} .\right.
$$

This is the first division of Theorem 1.5. By [K2, Lemma 4.5], it is easy to see that the identity double $X_{A B} \cup\left(-X_{A B}\right)$ (and also $\left.X_{C D} \cup\left(-X_{C D}\right)\right)$ is $S^{2} \times S^{2}$ or $S^{2} \tilde{\times} S^{2}$, depending on whether $P_{i}$ is even or odd. In such a sense, this theorem is the starting case of Problem 1.2. 


\subsection{Second sequences: Lens spaces with opposite orientations I}

Next, we recall a sequence $[S T U V]$ of pairs from Saito-Teragaito [ST], and define another (but similar) pairs [stuv], which give pairs of lens space surgeries along positive torus knots whose lens spaces are orientation-reversingly homeomorphic.

Definition 1.13 ([STUV] from $[\mathrm{ST}]$ and $[s t u v]$ )

We define a sequence of pairs $\left(S_{i}, T_{i}\right)$ and $\left(U_{i}, V_{i}\right)$ indexed by $i \geq 0$ as below.

$$
\left(\begin{array}{l}
S_{0} \\
T_{0}
\end{array}\right)=\left(\begin{array}{l}
0 \\
1
\end{array}\right), \quad\left(\begin{array}{l}
U_{0} \\
V_{0}
\end{array}\right)=\left(\begin{array}{l}
2 \\
1
\end{array}\right),
$$

and

$$
\left(\begin{array}{c}
S_{i+1} \\
T_{i+1}
\end{array}\right)=\left(\begin{array}{c}
T_{i} \\
S_{i}+V_{i}
\end{array}\right), \quad\left(\begin{array}{l}
U_{i+1} \\
V_{i+1}
\end{array}\right)=\left(\begin{array}{c}
V_{i} \\
T_{i}+U_{i}
\end{array}\right)
$$

inductively.

We define another sequence of pairs $\left(s_{i}, t_{i}\right)$ and $\left(u_{i}, v_{i}\right)$ indexed by $i \geq 0$ as below.

$$
\left(\begin{array}{l}
s_{0} \\
t_{0}
\end{array}\right)=\left(\begin{array}{l}
0 \\
1
\end{array}\right), \quad\left(\begin{array}{l}
u_{0} \\
v_{0}
\end{array}\right)=\left(\begin{array}{l}
1 \\
2
\end{array}\right)
$$

and

$$
\left(\begin{array}{c}
s_{i+1} \\
t_{i+1}
\end{array}\right)=\left(\begin{array}{c}
t_{i} \\
s_{i}+2 v_{i}
\end{array}\right), \quad\left(\begin{array}{l}
u_{i+1} \\
v_{i+1}
\end{array}\right)=\left(\begin{array}{c}
v_{i} \\
2 t_{i}+u_{i}
\end{array}\right)
$$

inductively.

From now on in this subsection, for each statement on $\left(\left(S_{i}, T_{i}\right),\left(U_{i}, V_{i}\right)\right)$ and $P_{i}$, it also holds on the other of pairs $\left(\left(s_{i}, t_{i}\right),\left(u_{i}, v_{i}\right)\right)$ and $p_{i}$.

Lemma 1.14 (See $[\mathrm{ST}]$ for $[S T U V])$ The pairs $\left(S_{i}, T_{i}\right)$ and $\left(U_{i}, V_{i}\right)$ satisfy the followings:

(1) Each pair $\left(S_{i}, T_{i}\right)$ and $\left(U_{i}, V_{i}\right)$ is coprime.

(2) $S_{i} T_{i}+1=U_{i} V_{i}-1$. We call this integer $P_{i}$.

(3) It holds that $S_{i}^{2}+V_{i}^{2} \equiv T_{i}^{2}+U_{i}^{2} \equiv 0 \bmod P_{i}$.

(4) If $i \geq 3$, then $S_{i}^{4} \not \equiv \pm 1 \bmod P_{i}$.

This lemma looks like Lemma 1.11 on $[A B C D]$ but differs at (3). By Lemma 1.14 (1)(3) and Moser's results, we have two sequences (from $[S T U V]$ and from [stuv]) of pairs of lens space surgeries along distinct torus knots whose lens spaces are orientation-reversingly homeomorphic:

$$
\begin{aligned}
& M\left(T\left(S_{i}, T_{i}\right) ; P_{i}\right) \cong-L\left(P_{i}, S_{i}{ }^{2}\right), \\
& M\left(T\left(U_{i}, V_{i}\right) ; P_{i}\right) \cong L\left(P_{i}, S_{i}{ }^{2}\right) .
\end{aligned}
$$

Thus we have a pair of 4-manifolds whose boundaries are orientation-reversingly homeomorphic:

$$
X_{S T}(i):=X\left(T\left(S_{i}, T_{i}\right) ; P_{i}\right), \quad X_{U V}(i):=X\left(T\left(U_{i}, V_{i}\right) ; P_{i}\right) .
$$


We often call them $X_{S T}, X_{U V}$ for short, respectively. We also define $X_{s t}=X_{s t}(i)$ and $X_{u v}=X_{u v}(i)$, by using the other sequence of pairs $\left(s_{i}, t_{i}\right)$ and $\left(u_{i}, v_{i}\right)$.

We postpone the 4-dim problem on $X_{S T} \cup X_{U V}$ and $X_{s t} \cup X_{u v}$ to the end of the next subsection, since the statement is almost same.

\subsection{Third sequences: Lens spaces with opposite orientations II}

We define two more sequences of pairs $[K L M N]$ satisfying $K L=M N$ and its similar one $[k l m n]$, which give pairs of lens space surgeries along positive torus knots whose lens spaces are orientation-reversingly homeomorphic.

Definition 1.15 ([KLMN] and $[k l m n])$

We define a sequence of pairs $\left(K_{i}, L_{i}\right)$ and $\left(M_{i}, N_{i}\right)$ indexed by $i \geq 0$ as below.

$$
\left(\begin{array}{l}
K_{0} \\
L_{0}
\end{array}\right)=\left(\begin{array}{l}
1 \\
3
\end{array}\right), \quad\left(\begin{array}{l}
M_{0} \\
N_{0}
\end{array}\right)=\left(\begin{array}{l}
1 \\
3
\end{array}\right)
$$

and

$$
\left(\begin{array}{c}
K_{i+1} \\
L_{i+1}
\end{array}\right)=\left(\begin{array}{cc}
1 & 0 \\
9 & -1
\end{array}\right)\left(\begin{array}{l}
M_{i} \\
N_{i}
\end{array}\right), \quad\left(\begin{array}{l}
M_{i+1} \\
N_{i+1}
\end{array}\right)=\left(\begin{array}{cc}
-1 & 1 \\
0 & 1
\end{array}\right)\left(\begin{array}{l}
K_{i} \\
L_{i}
\end{array}\right)
$$

We define a pair of the sequence $\left(k_{i}, l_{i}\right)$ and $\left(m_{i}, n_{i}\right)$ indexed by $i \geq 0$ as below.

$$
\left(\begin{array}{l}
k_{0} \\
l_{0}
\end{array}\right)=\left(\begin{array}{l}
1 \\
2
\end{array}\right), \quad\left(\begin{array}{l}
m_{0} \\
n_{0}
\end{array}\right)=\left(\begin{array}{l}
1 \\
2
\end{array}\right)
$$

and

$$
\left(\begin{array}{c}
k_{i+1} \\
l_{i+1}
\end{array}\right)=\left(\begin{array}{cc}
1 & 0 \\
8 & -1
\end{array}\right)\left(\begin{array}{c}
m_{i} \\
n_{i}
\end{array}\right), \quad\left(\begin{array}{c}
m_{i+1} \\
n_{i+1}
\end{array}\right)=\left(\begin{array}{cc}
-1 & 2 \\
0 & 1
\end{array}\right)\left(\begin{array}{c}
k_{i} \\
l_{i}
\end{array}\right) .
$$

We give an alternative definition, which looks a peculiarity of the third sequences.

Definition 1.16 (Alternative definitions of $[K L M N]$ and $[k l m n]$ ) Using a sequence $\left\{b_{i}\right\}$ defined by

$$
b_{0}=b_{1}=1, \quad b_{i+1}=3 b_{i}-b_{i-1}(i \geq 1),
$$

we define a pair $\left(\left(K_{i}, L_{i}\right),\left(M_{i}, N_{i}\right)\right)$ by

$$
\left(\left(K_{i}, L_{i}\right),\left(M_{i}, N_{i}\right)\right)=\left(\left(b_{i}, 3 b_{i+1}\right),\left(b_{i+1}, 3 b_{i}\right)\right) .
$$

Using a sequence $\left\{d_{i}\right\}$ defined by

$$
d_{0}=d_{1}=1, \quad d_{i+1}=4 d_{i}-d_{i-1}(i \geq 1),
$$

we define a pair $\left(\left(k_{i}, l_{i}\right),\left(m_{i}, n_{i}\right)\right)$ by

$$
\left(\left(k_{i}, l_{i}\right),\left(m_{i}, n_{i}\right)\right)=\left(\left(d_{i}, 2 d_{i+1}\right),\left(d_{i+1}, 2 d_{i}\right)\right) .
$$


Note that two definitions are equivalent. For $[K L M N]$, we have

$$
\begin{aligned}
& \left(\begin{array}{cc}
1 & 0 \\
9 & -1
\end{array}\right)\left(\begin{array}{l}
M_{i} \\
N_{i}
\end{array}\right)=\left(\begin{array}{cc}
1 & 0 \\
9 & -1
\end{array}\right)\left(\begin{array}{c}
b_{i+1} \\
3 b_{i}
\end{array}\right)=\left(\begin{array}{c}
b_{i+1} \\
9 b_{i+1}-3 b_{i}
\end{array}\right)=\left(\begin{array}{c}
b_{i+1} \\
3 b_{i+2}
\end{array}\right)=\left(\begin{array}{c}
K_{i+1} \\
L_{i+1}
\end{array}\right) \\
& \left(\begin{array}{cc}
-1 & 1 \\
0 & 1
\end{array}\right)\left(\begin{array}{l}
K_{i} \\
L_{i}
\end{array}\right)=\left(\begin{array}{cc}
-1 & 1 \\
0 & 1
\end{array}\right)\left(\begin{array}{c}
b_{i} \\
3 b_{i+1}
\end{array}\right)=\left(\begin{array}{c}
3 b_{i+1}-b_{i} \\
3 b_{i+1}
\end{array}\right)=\left(\begin{array}{c}
b_{i+2} \\
3 b_{i+1}
\end{array}\right)=\left(\begin{array}{c}
M_{i+1} \\
N_{i+1}
\end{array}\right) .
\end{aligned}
$$

We can check the equivalence for $[k l m n]$ in the similar way.

From now on in this subsection, for each statement on $\left(K_{i}, L_{i}\right),\left(M_{i}, N_{i}\right)$ and $P_{i}$, it also holds on the other sequence of pairs $\left(k_{i}, l_{i}\right),\left(m_{i}, n_{i}\right)$ and $p_{i}$.

Lemma 1.17 The pairs $\left(K_{i}, L_{i}\right)$ and $\left(M_{i}, N_{i}\right)$ satisfy the followings:

(1) Each pair $\left(K_{i}, L_{i}\right)$ and $\left(M_{i}, N_{i}\right)$ is coprime.

(2) $K_{i} L_{i}-1=M_{i} N_{i}-1$. We call this integer $P_{i}$.

(3) It holds that $K_{i}^{2}+M_{i}^{2} \equiv L_{i}^{2}+N_{i}^{2} \equiv 0 \bmod P_{i}$.

(4) If $i \geq 2$, then $K_{i}^{4} \not \equiv \pm 1 \bmod P_{i}$.

This lemma differs from Lemma 1.14 on $[S T U V]$ at the sign in (2). By Lemma 1.17 (1)-(3) and Moser's results, we have two sequences (from $[K L M N]$ and from $[k l m n]$ ) of pairs of lens space surgeries along distinct torus knots whose lens spaces are orientation-reversingly homeomorphic:

$$
\begin{aligned}
M\left(T\left(K_{i}, L_{i}\right) ; P_{i}\right) & \cong-L\left(P_{i}, K_{i}^{2}\right), \\
M\left(T\left(M_{i}, N_{i}\right) ; P_{i}\right) & \cong L\left(P_{i}, K_{i}{ }^{2}\right) .
\end{aligned}
$$

We have a pair of 4-manifolds whose boundaries are orientation-reversingly homeomorphic:

$$
X_{K L}(i):=X\left(T\left(K_{i}, L_{i}\right) ; P_{i}\right), \quad X_{M N}(i):=X\left(T\left(M_{i}, N_{i}\right) ; P_{i}\right) .
$$

We often call them $X_{K L}, X_{M N}$ for short, respectively. We also define $X_{k l}=X_{k l}(i)$ and $X_{m n}=X_{m n}(i)$, by using the other sequence $\left(\left(k_{i}, l_{i}\right),\left(m_{i}, n_{i}\right)\right)$ and $p_{i}$.

The main theorem on the 4-dim problem on $X_{S T} \cup X_{U V}$ and $X_{s t} \cup X_{u v}$ defined in the last subsection and those on $X_{K L} \cup X_{M N}$ and $X_{k l} \cup X_{m n}$ :

Theorem 1.18 Let $[X y \mathcal{W}]=\left(\left(X_{i}, y_{i}\right),\left(z_{i}, \mathcal{W}_{i}\right)\right)$ be one of the sequences

$$
[S T U V],[s t u v], \quad[K L M N],[k l m n]
$$

of pairs of pairs of integers. Let $X_{X y}=X_{X y}(i)$ and $X_{z \mathcal{W}}=X_{z \mathcal{W}}(i)$ be the 4-manifolds defined in the last and this subsection. For any $i \geq 1$, the smooth oriented 4-manifold $X_{X y} \cup X_{\mathcal{Z}}$ obtained by pasting $X_{X y}$ and $X_{z \mathcal{W}}$ by any orientation-reversing homeomorphism between their boundaries is diffeomorphic to $\mathbb{C} P^{2} \sharp \mathbb{C} P^{2}$ :

$$
X_{x y} \cup X_{z \mathcal{W}} \cong \mathbb{C} P^{2} \sharp \mathbb{C} P^{2} .
$$

This is the second division of Theorem 1.5. 


\begin{tabular}{c||c|c||c||c|c|}
\multicolumn{1}{c|}{$[A B C D](a=3)$} \\
\hline$i$ & $A$ & $B$ & $P$ & $C$ & $D$ \\
\hline 1 & 1 & 2 & 3 & 4 & 1 \\
\hline 2 & 4 & 5 & 21 & 11 & 2 \\
\hline 3 & 11 & 13 & 144 & 29 & 5 \\
\hline 4 & 29 & 34 & 378 & 76 & 13 \\
\hline 5 & 76 & 89 & 3117 & 199 & 34 \\
\hline$\vdots$ & & & $\vdots$ & & \\
\hline
\end{tabular}

\begin{tabular}{c||c|c||c||c|c|}
\multicolumn{1}{c|}{$[A B C D](a=4)$} \\
\hline$i$ & $A$ & $B$ & $P$ & $C$ & $D$ \\
\hline 1 & 1 & 3 & 4 & 5 & 1 \\
\hline 2 & 5 & 11 & 56 & 19 & 3 \\
\hline 3 & 19 & 41 & 780 & 71 & 11 \\
\hline 4 & 71 & 153 & 10864 & 265 & 41 \\
\hline 5 & 265 & 571 & 151316 & 989 & 153 \\
\hline$\vdots$ & & & $\vdots$ & & \\
\hline
\end{tabular}

Table 1: Sequence $((A, B),(C, D))$, indexed by integers $a$ and $i$

Corollary 1.19 We have four sequences of smooth embeddings of lens spaces into $\mathbb{C} P^{2} \sharp \mathbb{C} P^{2}$ : Let $(\mathcal{Z}, \mathcal{W})$ be one of the sequences $(U, V),(u, v),(M, N)$ and $(m, n)$ of pairs of integers defined in the last and this subsection. Let $P_{i}=Z_{i} \mathcal{W}_{i}-1$ for any choice. For any $i$, lens spaces $L\left(P_{i}, Z_{i}{ }^{2}\right)$ can be embedded smoothly in $\mathbb{C} P^{2} \sharp \mathbb{C} P^{2}$.

Recently, Sasahira [Sa] proved that some lens spaces can not be embedded into $\mathbb{C} P^{2} \sharp \mathbb{C} P^{2}$ as an application of his variation of instanton Floer homology. The authors (in an old version) of the present paper did know some examples of smooth embeddings of lens spaces into $\mathbb{C} P^{2} \sharp \mathbb{C} P^{2}$ as above. Proving the embedding of $L\left(P, Q^{\prime}\right)\left(P=28657, Q^{\prime}=7921\right.$, related to the pair $\left.\left(\left(S_{11}, T_{11}\right),\left(U_{11}, V_{11}\right)\right)=((199,144),(89,322))\right)$ referred in $[\mathrm{Sa}]$ is one of the purposes of the present paper.

This paper is organized as follows. In the next section, we define some notations and a certain two-component framed link $\left(T\left(\left(p_{z}, q_{z}\right),\left(p_{w}, q_{w}\right)\right) ; r_{z}, r_{w}\right)$. We also review on description of lens spaces and torus knots by chain framed links. In Section 3, we study the details on the sequences of the pairs of lens space surgeries along torus knots. This is a number-theoretic part. In Section 4, we do 3-dim calculus on chain framed links to get the 4-dim diagrams of the constructed 4-manifolds. They depend on the choice of the sequences and the indices. In Section 5, we will prove the diffeomorphism in Theorem 1.5 from the constructed manifolds to the standard manifolds by 4-dim calculus. All process (diagrams and calculus) depend on the indices $i$. In Section 6, we will prove Theorem 1.4. This is a non-geometric part. The five sequences are mutually similar but different. The proofs are divided into two or three subsections (cases) depending the similarity. 


\begin{tabular}{|c||c|c||c||c|c|}
\multicolumn{1}{c|}{$[$ STUV] } \\
\hline$i$ & $S$ & $T$ & $P$ & $U$ & $V$ \\
\hline 1 & 1 & 1 & 2 & 1 & 3 \\
\hline 2 & 1 & 4 & 5 & 3 & 2 \\
\hline 3 & 4 & 3 & 13 & 2 & 7 \\
\hline 4 & 3 & 11 & 34 & 7 & 5 \\
\hline 5 & 11 & 8 & 89 & 5 & 18 \\
\hline 6 & 8 & 29 & 233 & 18 & 13 \\
\hline$\vdots$ & & & $\vdots$ & & \\
\hline
\end{tabular}

\begin{tabular}{|c||c|c||c||c|c|}
\multicolumn{7}{c|}{$[$ stuv $]$} \\
\hline$i$ & $s$ & $t$ & $p$ & $u$ & $v$ \\
\hline 1 & 1 & 4 & 5 & 2 & 3 \\
\hline 2 & 4 & 7 & 29 & 3 & 10 \\
\hline 3 & 7 & 24 & 169 & 10 & 17 \\
\hline 4 & 24 & 41 & 985 & 17 & 58 \\
\hline 5 & 41 & 140 & 5741 & 58 & 99 \\
\hline 6 & 140 & 239 & 33461 & 99 & 338 \\
\hline$\vdots$ & & & $\vdots$ & & \\
\hline
\end{tabular}

Table 2: Sequences $((S, T),(U, V))$ and $((s, t),(u, v))$, indexed by integers $i$

$[K L M N]$

\begin{tabular}{|c||c|c||c||c|c|}
\hline$i$ & $K$ & $L$ & $P$ & $M$ & $N$ \\
\hline 1 & 1 & 6 & 5 & 2 & 3 \\
\hline 2 & 2 & 15 & 29 & 5 & 6 \\
\hline 3 & 5 & 39 & 194 & 13 & 15 \\
\hline 4 & 13 & 102 & 1325 & 34 & 39 \\
\hline 5 & 34 & 267 & 9077 & 89 & 102 \\
\hline$\vdots$ & & & $\vdots$ & & \\
\hline
\end{tabular}

Table 3: Sequences $((K, L),(M, N))$ and $((k, l),(m, n))$, indexed by integers $i$ $[k \operatorname{lm} n]$

\begin{tabular}{|c||c|c||c||c|c|}
\hline$i$ & $k$ & $l$ & $p$ & $m$ & $n$ \\
\hline 1 & 1 & 6 & 5 & 3 & 2 \\
\hline 2 & 3 & 22 & 65 & 11 & 6 \\
\hline 3 & 11 & 82 & 901 & 41 & 22 \\
\hline 4 & 41 & 306 & 12545 & 153 & 82 \\
\hline 5 & 153 & 1142 & 174725 & 571 & 306 \\
\hline$\vdots$ & & & $\vdots$ & & \\
\hline
\end{tabular}

(1)

\section{Preliminaries}

\subsection{Strategy of the proof}

Our strategy of the proof of Theorem 1.5 is (1) turning upside down and (2) describing dual knots of lens space surgery. Here, we consider a 4-manifold $X_{1} \cup_{\varphi}\left(-X_{2}\right)$ in the case where $X_{1}=X\left(K_{1} ; n\right), X_{2}=X\left(K_{2} ; n\right)$ whose boundaries are orientation-preservingly homeomorphic: $M\left(K_{1} ; n\right) \cong M\left(K_{2} ; n\right)$.

(1) The method turn upside down is explained in Gompf-Stipsicz's graduate text [GS, p.130 and $\S 5.5]$ as an application of taking the identity double of a manifold in Kirby [K2, Lemma 4.5]. For recent use, see Akbulut's [A] and so on. Each 4-manifold $X_{1}$ and $X_{2}$ admits a handle decomposition consisting of a 0 -handle and a 2-handle:

$$
X_{1}=h_{1}^{0} \cup h_{1}^{2}, \quad X_{2}=h_{2}^{0} \cup h_{2}^{2},
$$

where $h^{r}$ means an $r$-handle. The 2 -handle $h_{i}^{2}$ is attached to $h_{i}^{0}$ along $K_{i}$ with $n$-framing $(i=1,2)$. To construct $X_{1} \cup_{\varphi}\left(-X_{2}\right)$, we turn $X_{2}$ upside down (reverse the orientation) and glue it along the common boundary by an orientation-reversing homeomorphism. By turning upside down, the attaching part $\left(\partial D^{r} \times D^{4-r}\right)$ and the boundary part $\left(D^{r} \times \partial D^{4-r}\right)$ 
of an $r$-handle $h^{r}$ are interchanged. We denote the latter $(4-r)$-handle by $\left(h^{r}\right)^{\perp}$ and call it dualized handle of $h^{r}$. The manifold $X_{1} \cup_{\varphi}\left(-X_{2}\right)$ admits a handle decomposition consisting of a 0-handle, two 2-handles and a 4-handle:

$$
X_{1} \cup_{\varphi}\left(-X_{2}\right)=h_{1}^{0} \cup h_{1}^{2} \cup\left(h_{2}^{2}\right)^{\perp} \cup\left(h_{2}^{0}\right)^{\perp} .
$$

We realize the homeomorphism $\varphi$ by 3 -dim calculus from $\left(K_{2} ; n\right)$ to $\left(K_{1} ; n\right)$. The second 2-handle $\left(h_{2}^{2}\right)^{\perp}$ is attached along the result of 0 -framed meridian of $K_{2}$ under the realization of $\varphi$.

(2) To show 4-dim diagram description of $X_{1} \cup_{\varphi}\left(-X_{2}\right)$, we search dual knots of the lens space surgeries from the view point of 3-dim topology. Let $M(K ; n)=E(K) \cup V=L(p, q)$ be a lens space surgery, where $E(K)$ is the exterior of $K$ in $S^{3}$ and $V$ is a solid torus attached to $E(K)$ such that a meridian in $\partial V$ maps to the $n$-framing of $K$ in $\partial E(K)$. Considering its reversed surgery, i.e., cutting $V$ back from $L(p, q)$ and gluing it to construct the initial $S^{3}$, we call the core curve of $V$ in $L(p, q)$ dual knot of the lens space surgery. Instead of framings, a dual knot has a surgery slope, i.e., an isotopy class of a simple closed curve in $\partial(L(p, q) \backslash \operatorname{int} V)$ is specified. Suppose that $L(p, q)$ is a result of a lens space surgery and is described by a diagram in $S^{3}$. Then the dual knot with a surgery slope is described in the diagram as a framed knot. For a pair of the lens space surgeries along distinct knots, we will describe both dual knots in a diagram of the common resulting lens space.

\subsection{Notations}

Kirby calculus consists of (K-I) handle-slides, and (K-II) blow-ups, blow-downs and their orientation-reversed versions. A handle-slide (K-I) keeps the 4-manifold and its boundary. A Kirby move in (K-II) keeps the boundary, but changes the 4-manifold. Thus, when we prove a diffeomorphism between 4-manifolds, we use only (K-I). We call such a calculus 4-dim calculus and use a simple arrow, see Figure 26. On the other hand, when we are concerned with the boundary (for example, when we search attaching circles), we can use both (K-I) and (K-II). We call such a calculus 3 -dim calculus and use arrow with a symbol " $\partial$ ", see Figure 1.

Most 3-dim diagrams in this paper are framed chain links. We use the usual labeled graph description of chained framed links and its basic formulas in Figure 1, where $x, y, n$ are integers, and $r$ can be a rational number. We often use (nontrivial) diagrams of $S^{3}$. When a labeled graph describes $S^{3}$, we use a dotted line (see Figure 5). We also use another formula,

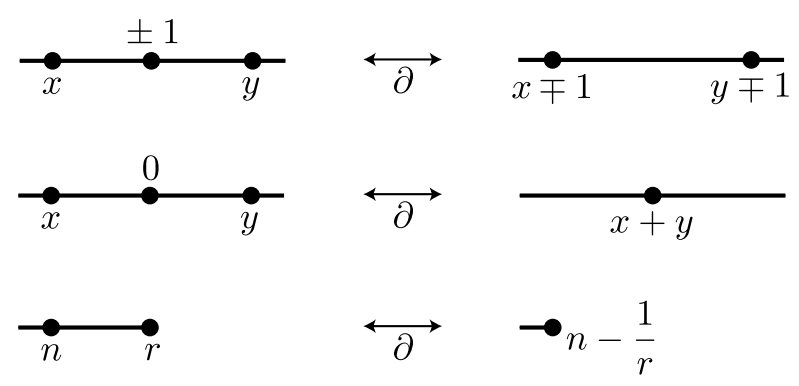

Figure 1: Basic formulas in 3-dim calculus 

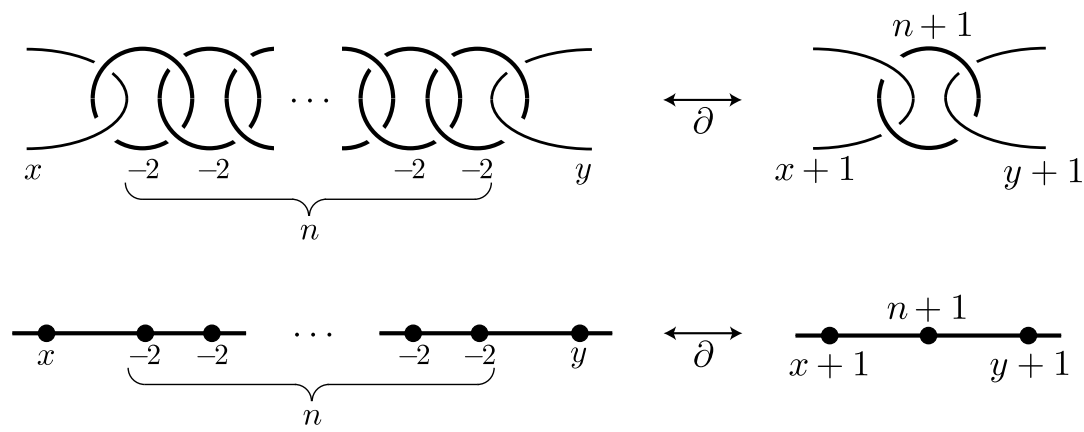

Figure 2: $(-2)$ s formula $(n \geq 1)$

which we call $(-2)$-formula, in Figure 2.

Next, we define some notations of two-component links of special type. We start with the positively linking Hopf link. We call its two components $z$ and $w$, see the left picture in Figure 3. We take their regular neighborhoods and call their boundaries $T_{z}$ and $T_{w}$, respectively. In the torus $T_{z}$ (and $T_{w}$, respectively), we take an oriented meridian-longitude system $\left(m_{z}, l_{z}\right)$ (and $\left(m_{w}, l_{w}\right)$ ) as in the right figure in Figure 3. Note that $m_{z}$ is isotopic to $l_{w}$, and that $l_{z}$ is isotopic to $m_{w}$, in the complement of $z \cup w$ in $S^{3}$.
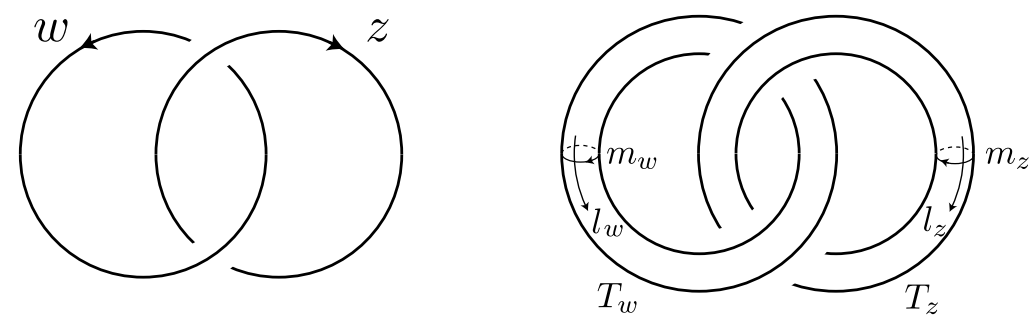

Figure 3: Hopf Link and $T\left(\left(p_{z}, q_{z}\right),\left(p_{w}, q_{w}\right)\right)$

Definition 2.1 By $T\left(\left(p_{z}, q_{z}\right),\left(p_{w}, q_{w}\right)\right)$, we denote a two-component link

$$
T\left(\left(p_{z}, q_{z}\right),\left(p_{w}, q_{w}\right)\right):=T_{z}\left(p_{z}, q_{z}\right) \cup T_{w}\left(q_{w}, p_{w}\right),
$$

where $T_{z}\left(p_{z}, q_{z}\right)$ is a knot in $T_{z}$ whose homology class is $p_{z}\left[l_{z}\right]+q_{z}\left[m_{z}\right]$, and $T_{w}\left(p_{w}, q_{w}\right)$ is a knot in $T_{w}$ whose homology class is $q_{w}\left[l_{w}\right]+p_{w}\left[m_{w}\right]$. See an example $T((5,11),(1,3))$ in Figure 25. In particular, each component is a torus knot. Their linking number is $p_{z} q_{w}$.

Lemma 2.2 There is a symmetry $T\left(\left(q_{w}, p_{w}\right),\left(q_{z}, p_{z}\right)\right)=T\left(\left(p_{z}, q_{z}\right),\left(p_{w}, q_{w}\right)\right)$, i.e., there exists a self diffeomorphism $\Phi$ of $S^{3}$ that changes $T\left(\left(p_{z}, q_{z}\right),\left(p_{w}, q_{w}\right)\right)$ to $T\left(\left(q_{w}, p_{w}\right),\left(q_{z}, p_{z}\right)\right)$. It extends to an equivalence of the framed links between $\left(T\left(\left(p_{z}, q_{z}\right),\left(p_{w}, q_{w}\right)\right) ; r_{z}, r_{w}\right)$ and $\left(T\left(\left(q_{w}, p_{w}\right),\left(q_{z}, p_{z}\right)\right) ; r_{w}, r_{z}\right)$. 


\subsection{Framed chain links, Lens spaces and Torus knots}

Moser's proof in [Mo] of lens space surgeries along torus knots (Lemma 1.3) is from the view point of Seifert fibered structure. Here we reprove Lemma 1.3 by using framed chain links using the algorithm defined in [Y1, Y2], to search dual knots better.

Framed chain link description of a lens space $L(p, q)$ using continued fraction expansion is well-known: If $p / q=\left[x_{1}, x_{2}, \cdots, x_{n}\right], L(p, q)$ is described by the framed chain link whose

$$
\left[x_{1}, x_{2}, x_{3}, \cdots, x_{n}\right]=x_{1}-\frac{1}{x_{2}-\frac{1}{x_{3}-\ddots-\frac{1}{x_{N}}} .}
$$

Figure 4: Lens space $\left(p / q=\left[x_{1}, x_{2}, \cdots, x_{n}\right]\right)$

coefficients are $-x_{1},-x_{2}, \cdots,-x_{n}$. This is our notation and orientation of lens spaces. Note that if $p>q>0$, the continued fraction expansion $p / q=\left[x_{1}, x_{2}, \cdots, x_{n}\right]$ with all $x_{i}>1$ is unique. Let $\bar{q}$ be the unique integer satisfying $q \bar{q} \bmod p$ and $0<\bar{q}<p$, then $p / \bar{q}=$ $\left[x_{n}, \cdots, x_{2}, x_{1}\right]$ (i.e., the reversed order). Lens spaces $L(p, q)$ and $L\left(p, q^{\prime}\right)$ are orientationpreservingly homeomorphic to each other if and only if " $q^{\prime} \equiv q$ or $q^{\prime} \equiv \bar{q} \bmod p$ ". As a construction of lens space by a rational Dehn surgery along the Hopf link, the following is also known:

Proposition 2.3 Dehn surgery along the Hopf link with coefficients $\alpha_{1} / \beta_{1}, \alpha_{2} / \beta_{2}$ is $L(P, Q)$ with $P=\alpha_{1} \alpha_{2}-\beta_{1} \beta_{2}, Q=\alpha_{1} \gamma_{2}-\beta_{1} \delta_{2}$, where $\gamma_{2}, \delta_{2}$ are the integers satisfying $\alpha_{2} \delta_{2}-\beta_{2} \gamma_{2}=1$.

Next, we study description of torus knots by framed chain links. For a coprime pair $(p, q)$ of positive integers with $p, q>1$, we take another unique coprime pair $(r, s)$ of positive integers satisfying

$$
p s-q r=1
$$

and $0<r<p, 0<s<q$ (thus $\bar{p}=s \bmod q$ and $\bar{q}=p-r \bmod p$ ). Then, the torus knot $T(p, q)$ and Moser's lens space surgeries (Lemma 1.3) can be described as follows: See the framed chain link in Figure 5 (a component $z$ will be referred later in Lemma 2.7), where the coefficients $c_{i}$ are determined by Algorithm below, and satisfy the equalities:

$$
\frac{q}{q-s}=\left[c_{-1}, c_{-2}, \cdots, c_{-n_{R}}\right], \quad \frac{p}{r}=\left[c_{1}, c_{2}, \cdots, c_{n_{L}}\right] .
$$

\footnotetext{
Algorithm ([Y1, Y2])
} 

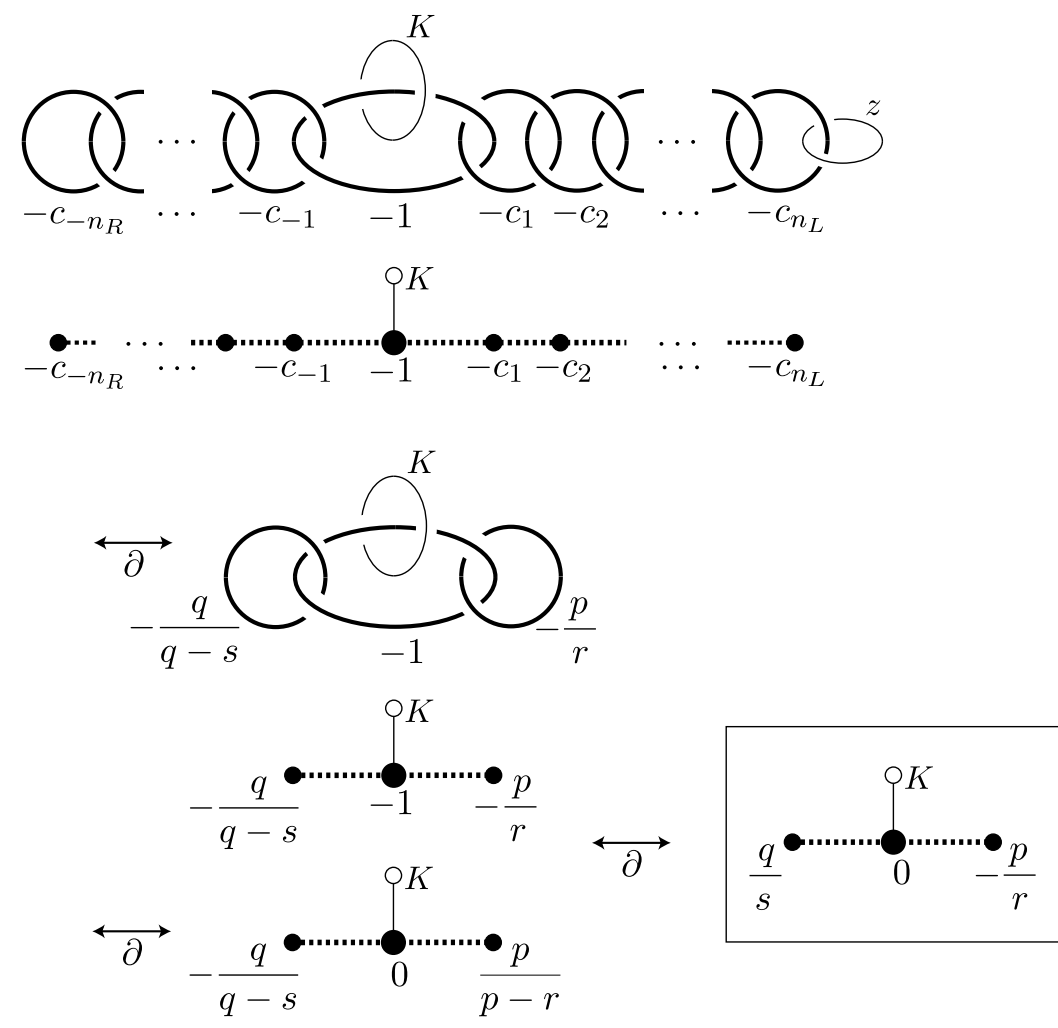

Figure 5: Torus knot $T(p, q)$ is presented by Diagram $\mathcal{D}(p, q)(p s-q r=1)$

(1) Euclidean algorithm: From the pair $(p, q)$, we construct a word $w(p, q)=w_{1} w_{2} \cdots w_{N}$ of two letters $L$ (left) and $R$ (right) inductively by the rule below:

Start with $\left(p_{0}, q_{0}\right):=(p, q)$.

$$
\begin{aligned}
& \text { ILR Rule] } p_{i}>q_{i}, \quad \text { then } w_{i+1}:=L \text { and }\left(p_{i+1}, q_{i+1}\right):=\left(p_{i}-q_{i}, q_{i}\right) . \\
& \text { If } p_{i}<q_{i}, \quad \text { then } w_{i+1}:=R \text { and }\left(p_{i+1}, q_{i+1}\right):=\left(p_{i}, q_{i}-p_{i}\right) .
\end{aligned}
$$

By coprimeness of $(p, q)$, after some $N$ steps, the pair $\left(p_{N}, q_{N}\right)$ becomes to $(1,1)$, which is the end of this step. We define $n_{L}$ (and $n_{R}$, respectively) as the number of $L$ (and $R$ ) in the word $w(p, q)$. Thus $n_{L}+n_{R}=N$.

(2) Next, starting with a single (-1)-labeled vertex $((-1)$-vertex, for short) in a horizontal short edge, we iterate blow-ups $N$ times guided by the words in $w(p, q)$. In the $i$-th step (the $i$-th blow-up), we do the operation $L$ (or $R$, respectively) on the diagram near the $(-1)$-vertex according to $w_{i}=L$ (or $R$ ) as in Figure 6, see also the example in Figure 7. We have a diagram of a framed chain link. We call it long diagram $\mathcal{D}(p, q)$, where we use a dotted line, since it describes $S^{3}$.

(3) We define $c_{i}$ (with $-n_{R} \leq i \leq n_{L}$ ) as the absolute value of the label of the $i$-th vertex (the framing of the $i$-th component) from the left in the resulting framed chain link, where we regard the finally appeared $(-1)$-vertex as the 0 -th vertex. The coefficients $c_{i}$ s satisfy the 


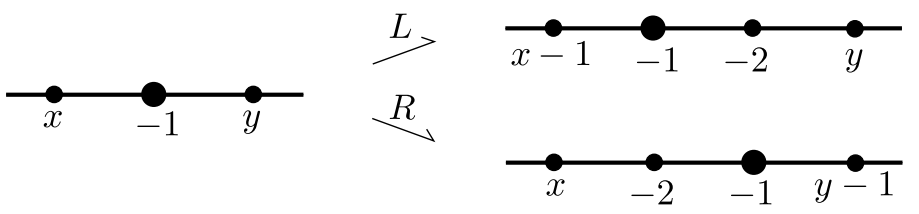

Figure 6: Operation $L$ and $R$

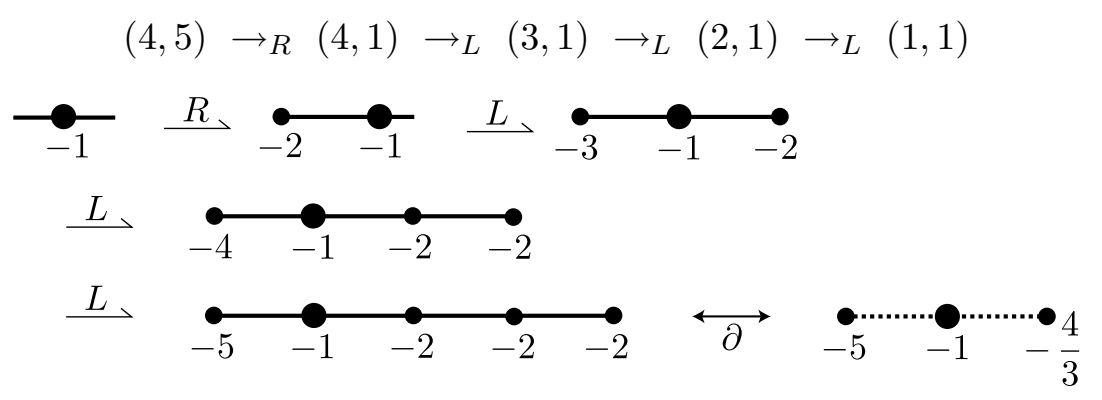

Figure 7: Example $T(4,5) \quad(w(4,5)=R L L L)$

equation (2.2). By the basic formulas in Figure 1, we can shorten the diagram $\mathcal{D}(p, q)$ as in Figure 5. In the final deformations, we used equalities

$$
\left[-1,-\frac{q}{q-s}\right]=-1+\frac{1}{\frac{q}{q-s}}=-\frac{s}{q}=\left[0, \frac{q}{s}\right], \quad\left[-1,-\frac{p}{r}\right]=\frac{r-p}{p}=\left[0, \frac{p}{p-r}\right] .
$$

The second deformation is related to a switch from $(p, q)$ to $(q, p)$. Note that $q(p-r)-p(q-s)=$ 1 by the equation (2.1).

The second step (2) consists of only blow-ups. It is a 3 -dim calculus, thus $\mathcal{D}(p, q)$ (ignoring $K)$ in Figure 5 describes $S^{3}$. By the second author's method [Y1, Y2], we can prove the integral lens space surgery along a torus knot by framed chain links. This is an alternative proof of (a part of) the Moser's results [Mo].

Lemma 2.4 Let $(p, q)$ be a coprime pair of positive integers. We take another pair $(r, s)$ satisfying ps $-r q=1,0<r<p, 0<s<q$. As description of 3-manifolds, the followings hold.

(1) Diagram $\mathcal{D}(p, q)$ in Figure 5 describes $S^{3}$.

(2) In the resulting $S^{3}$ in (1) above, the component $K$ is the torus $k n o t T(p, q)$.

(3) Furthermore, $n$-framing of $K$ in (1) becomes $(p q+n)$-framing of $T(p, q)$ in $S^{3}$.

(4) Considering the total components $\mathcal{D}(p, q) \cup(K ; \pm 1)$, we have the lens space surgery $M(T(p, q) ; p q \pm 1) \cong-L\left(p q \pm 1, p^{2}\right)$, see Figure 9 (ignoring $\left.K^{*}\right)$.

Proof. (1) has been already proved. For (2) and (3), see [Y2]. (4) follows from a 3-dim calculus in Figure 8 and by Proposition 2.3. 


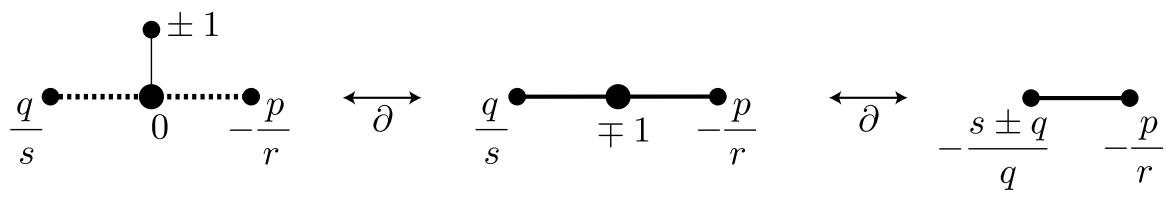

Figure 8: Lens space surgery along $T(p, q)(p s-q r=1)$

From the viewpoint of 4-dim topology, $\mathcal{D}(p, q) \cup(K ; \pm 1)$ can be regarded as a relative Kirby diagram of the (4,3)-dim cobordism $\left(X, \partial_{-} X \cup \partial_{+} X\right)$ from $\partial_{-} X=S^{3}$ described by $\mathcal{D}(p, q)$ to $\partial_{+} X=-L\left(p q \pm 1, p^{2}\right)$ obtained by 2 -handle attaching along $K$. By $\mathcal{L}(p, q)$, we denote the diagram describing $-L\left(p q \pm 1, p^{2}\right)$ at the center of Figure 8. Using this, we can find the dual knot of the lens space surgery. For any Dehn surgery along a component $k$, its 0 -framed meridian cancels the surgery, which is the reversed surgery. Figure 9 is obtained from the first half of Figure 8 by giving the reversed surgery to $K$ in $\mathcal{D}(p, q) \cup(K ; \pm 1)$. Thus we have:

Lemma 2.5 The dual knot of the lens space surgery in Lemma 2.4(4) is the component $K^{*}$ in Figure 9, whose framings are written in the parenthesis.

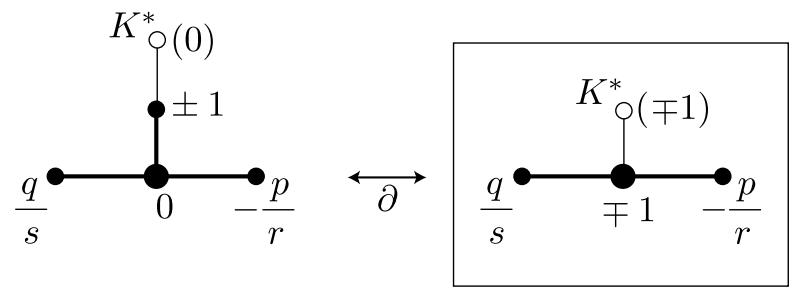

Figure 9: Diagram $\mathcal{L}(p, q)$ with $K^{*}$

Definition 2.6 We call the operation from $\mathcal{D}(p, q) \cup(K ; \pm 1)$ (boxed one in Figure 5) to $\mathcal{L}(p, q) \cup\left(K^{*} ; \mp 1\right)$ (Figure 9) dualization of $K$. We also call the reversed operation from $\mathcal{L}(p, q) \cup\left(K^{*} ; \mp 1\right)$ to $\mathcal{D}(p, q) \cup(K ; \pm 1)$, dualization of $K^{*}$, in the sense $\left(K^{*}\right)^{*}=K$.

It corresponds to turning upside down $\left(\bar{X}, \partial_{-} \bar{X} \cup \partial_{+} \bar{X}\right)$ of a relative Kirby diagram $\left(X, \partial_{-} X \cup\right.$ $\partial_{+} X$ ) (in the sense of [GS, $\left.\S 5.5\right]$ ), where $\bar{X}$ is the orientation-reversed $X$ with $\partial_{ \pm} \bar{X}=-\partial_{\mp} X$.

Finally, we study Heegaard torus in lens spaces (or $S^{1} \times S^{2}, S^{3}$ ). Heegaard torus of such a manifold is known to be unique up to ambient isotopy. See diagram in Figure 10, which consists of an $n$-component framed chain link $K_{1} \cup K_{2} \cup \ldots \cup K_{n}$ in $S^{3}$ and the sequence of solid tori $V_{1} \subset V_{2} \subset \cdots \subset V_{n}$. The solid torus $V_{i}$ satisfies that $K_{j} \subset \operatorname{int} V_{i}$ if $j \leq i$ and that $K_{j} \cap V_{i}=\emptyset$ if $j>i$. By $T_{i}$, we denote the boundary of the solid torus $V_{i}$. Then we have:

Lemma 2.7 In the resulting lens space (or $S^{1} \times S^{2}, S^{3}$ ), each $T_{i}$ is a Heegaard torus. Thus all $T_{i}$ s are mutually parallel. Suppose that the resulting manifold is $S^{3}$. Then, we have

(1) Each meridian $m_{i}$ of $K_{i}$ is a torus knot in the resulting $S^{3}$. 


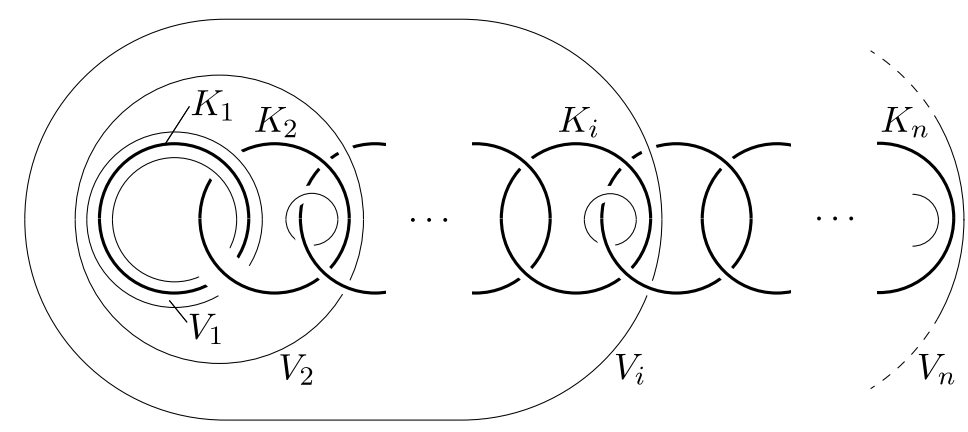

Figure 10: Solid tori

(2) (Precise version of Lemma 2.4(2)) In the special case $\mathcal{D}(p, q)$ in Figure $5, K$ is the torus $k n o t T(p, q)$ in the torus $T_{z}$ (It is equivalent to $T(q, p)$ in $T_{w}$ ).

(3) A union $m_{i} \cup m_{j}$ of a pair of meridians of $K_{i} \cup K_{j}$ is a link of type $T\left(\left(p_{z}, q_{z}\right),\left(p_{w}, q_{w}\right)\right)$ in the resulting $S^{3}$. Types $\left(p_{z}, q_{z}\right)$ and $\left(p_{w}, q_{w}\right)$ are calculated by deforming the diagram and applying (2) above.

See Definition 2.1 for the notations $T_{z}, T_{w}$ and $T\left(\left(p_{z}, q_{z}\right),\left(p_{w}, q_{w}\right)\right)$.

\subsection{Contribution of pasting maps}

For Problem 1.1, we have to study the contribution of the pasting maps between the boundaries of the pieces. The diffeotopy groups $\pi_{0}\left(\operatorname{Difff}^{+}(L(p, q))\right)$ of lens spaces $L(p, q)$ are studied by Bonahon [Bon] and Hodgson-Rubinstein [HR]:

Proposition 2.8 ([Bon, p.307], [HR, p.89])

If $q^{2} \not \equiv \pm 1$ or $q \equiv \pm 1 \bmod p, \pi_{0}\left(\operatorname{Diff}^{+}(L(p, q))\right) \cong \mathbb{Z} / 2 \mathbb{Z}$. The generator is the $\pi$-rotation map in Figure 11, derived from $\rho(z, w)=(\bar{z}, \bar{w})$. In the other case, $\pi_{0}\left(\operatorname{Diff}^{+}(L(p, q))\right) \cong \mathbb{Z} / 4 \mathbb{Z}$ $\left(p \neq 2\right.$ and $\left.q^{2} \equiv-1 \bmod p\right)$, or $\cong(\mathbb{Z} / 2 \mathbb{Z})^{2}\left(q \not \equiv \pm 1\right.$ and $\left.q^{2} \equiv 1 \bmod p\right)$.

For any sequences $[X y z \mathcal{W}]$ in

$$
[A B C D], \quad[S T U V],[s t u v], \quad[K L M N],[k l m n],
$$

it holds that the resulting lens space $-L\left(P, X^{2}\right)$ satisfies $X^{4} \not \equiv \pm 1(\bmod P)$, in the properties (4) in Lemma 1.11, 1.14 or 1.17, which will be proved in Section 3. Thus, by Proposition 2.8, we have only two choices on the pasting map: the identity map or the involution generator. In Section 4, we will prove that the pair of dual knots can be taken as a union of a pair of meridians of a framed chain link of the lens space, as in Figure 12, where $c \in \mathbb{Z}, \delta_{1}, \delta_{2} \in\{ \pm 1\}$ and $r_{1}, r_{2} \in \mathbb{Q}$. Each meridian is kept either by the identity map or by the involution. Thus, the choice of the pasting maps does not contribute the resulting closed 4-manifold.

\section{Formulas on the sequences}

We prove Lemma 1.11, Lemma 1.14 and Lemma 1.17. They are on the pairs of pairs of integers in the sequences. 

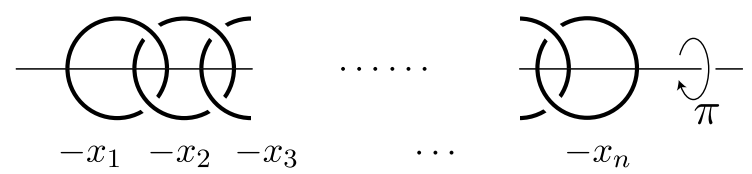

Figure 11: An involution on $L(p, q)$
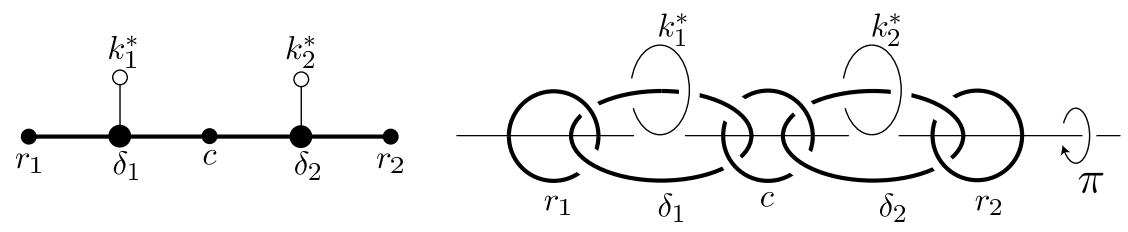

Figure 12: Pair of dual knots in the lens space

\subsection{Formulas on the first sequence}

First, for a fixed $a$, we write down the pairs in the case $i=1,2,3$ :

$$
\begin{aligned}
\left(\begin{array}{l}
A_{1} \\
B_{1}
\end{array}\right)=\left(\begin{array}{c}
1 \\
a-1
\end{array}\right), & \left(\begin{array}{l}
C_{1} \\
D_{1}
\end{array}\right)=\left(\begin{array}{c}
a+1 \\
1
\end{array}\right), \\
\left(\begin{array}{l}
A_{2} \\
B_{2}
\end{array}\right)=\left(\begin{array}{c}
a+1 \\
a^{2}-a-1
\end{array}\right), & \left(\begin{array}{c}
C_{2} \\
D_{2}
\end{array}\right)=\left(\begin{array}{c}
a^{2}+a-1 \\
a-1
\end{array}\right), \\
\left(\begin{array}{l}
A_{3} \\
B_{3}
\end{array}\right)=\left(\begin{array}{c}
a^{2}+a-1 \\
a^{3}-a^{2}-2 a+1
\end{array}\right), & \left(\begin{array}{l}
C_{3} \\
D_{3}
\end{array}\right)=\left(\begin{array}{c}
a^{3}+a^{2}-2 a-1 \\
a^{2}-a-1
\end{array}\right) .
\end{aligned}
$$

They also satisfy

$$
\left(\begin{array}{l}
A_{i+2} \\
B_{i+2}
\end{array}\right)=\left(\begin{array}{cc}
1 & a+2 \\
a-2 & a^{2}-3
\end{array}\right)\left(\begin{array}{l}
A_{i} \\
B_{i}
\end{array}\right), \quad\left(\begin{array}{c}
C_{i+2} \\
D_{i+2}
\end{array}\right)=\left(\begin{array}{cc}
a^{2}-3 & a+2 \\
a-2 & 1
\end{array}\right)\left(\begin{array}{c}
C_{i} \\
D_{i}
\end{array}\right) .
$$

The proof of Lemma 1.11 is started with another set of formulas on the sequences $\left(A_{i}, B_{i}\right)$ and $\left(C_{i}, D_{i}\right)$ :

Lemma 3.1 The pairs $\left(A_{i}, B_{i}\right)$ and $\left(C_{i}, D_{i}\right)$ satisfy the followings:

(1) $A_{i}+B_{i}-C_{i}+D_{i}=0$.

(2) $(a-1) A_{i}-B_{i}-C_{i}+(a+1) D_{i}=0$.

(3) Each of $A_{i}, B_{i}, C_{i}$ and $D_{i}$ satisfies the same recursive formula:

$$
X_{i+2}=a X_{i+1}-X_{i}, \quad \text { for } X=A, B, C \text { or } D .
$$

(4) It holds that $A_{i} \equiv D_{i}\left(=: \mathfrak{a}_{i}\right)$ and $B_{i} \equiv C_{i}\left(=: \mathfrak{b}_{i}\right) \bmod 2$. Furthermore, if a is even then $\mathfrak{a}_{i} \equiv \mathfrak{b}_{i} \equiv 1$ for every $i$. Otherwise, $\left(\mathfrak{a}_{i}, \mathfrak{b}_{i}\right) \equiv(1,1),(1,0),(0,1)$ according to $i \equiv 0,1,2$ $\bmod 3$, respectively. 
(5) For any pair $(X, y)$ in $\{A, B, C, D\}, x_{i+1} y_{i}-y_{i+1} x_{i}$ is constant, i.e., does not depend on $i$. For example, $A_{i+1} B_{i}-B_{i+1} A_{i}=a$ and $A_{i+1} D_{i}-D_{i+1} A_{i}=2$.

Proof of Lemma 3.1(1) and (2). In the case $i=1$, we can check it directly. By the inductive definitions in (1.1), we have

$$
\begin{aligned}
A_{i+1}+ & B_{i+1}-C_{i+1}+D_{i+1} \\
= & C_{i}+(a-2) C_{i}+D_{i}-A_{i}-(a+2) B_{i}+B_{i} \\
= & -a\left(A_{i}+B_{i}-C_{i}+D_{i}\right) \\
& \quad+(a-1) A_{i}-B_{i}-C_{i}+(a+1) D_{i},
\end{aligned}
$$

and

$$
\begin{aligned}
& (a-1) A_{i+1}-B_{i+1}-C_{i+1}+(a+1) D_{i+1} \\
& \quad=(a-1) C_{i}-(a-2) C_{i}-D_{i}-A_{i}-(a+2) B_{i}+(a+1) B_{i} \\
& \quad=-\left(A_{i}+B_{i}-C_{i}+D_{i}\right) .
\end{aligned}
$$

Lemma 3.1(1) and (2) are proved simultaneously by induction with the formulas above.

Proof of Lemma 3.1(3), (4) and (5).

$$
\begin{aligned}
A_{i+2} & =C_{i+1}=A_{i}+a B_{i}+2 B_{i} \\
& =A_{i}+a\left(-A_{i}+C_{i}-D_{i}\right)+2 B_{i} \\
& =a C_{i}-\left\{(a-1) A_{i}+(a+1) D_{i}\right\}+D_{i}+2 B_{i} \\
& =a C_{i}-\left(B_{i}+C_{i}\right)+D_{i}+2 B_{i} \\
& =a C_{i}+B_{i}-C_{i}+D_{i} \\
& =a A_{i+1}-A_{i},
\end{aligned}
$$

where we used Lemma 3.1(1) twice and (2) once. Since $D_{i}=A_{i+1}$, the sequence $D_{i}$ has the same recursive formula as that for $A_{i}$. By Lemma 3.1(1) and (2), both $B_{i}$ and $C_{i}$ are linear combination of $A_{i}$ and $D_{i}$. Thus, they also have the same recursive formula. Lemma 3.1(4),(5) follow from (3). For (5), we take the determinants of

$$
\left(\begin{array}{ll}
x_{i+2} & x_{i+1} \\
y_{i+2} & y_{i+1}
\end{array}\right)=\left(\begin{array}{ll}
x_{i+1} & x_{i} \\
y_{i+1} & y_{i}
\end{array}\right)\left(\begin{array}{cc}
a & 1 \\
-1 & 0
\end{array}\right)
$$

Now we prove Lemma 1.11 in Section 1.

Proof of Lemma 1.11(1). It follows from that

$$
\left(\begin{array}{cc}
1 & 0 \\
a-2 & 1
\end{array}\right),\left(\begin{array}{cc}
1 & a+2 \\
0 & 1
\end{array}\right) \in S L(2 ; \mathbb{Z})
$$

Proof of Lemma 1.11(2). It follows from the equation $A_{i+1} D_{i}-D_{i+1} A_{i}=2$ in Lemma 3.1(5) and $A_{i+1}=C_{i}, D_{i+1}=B_{i}$ in the recursive definition (1.1) in Section 1.

Proof of Lemma 1.11(3). We start with 
Claim 1. $P_{i+1}=P_{i}+(a-2) C_{i}^{2}+2$.

Proof.

$$
\begin{aligned}
P_{i+1} & =A_{i+1} B_{i+1}+1 \\
& =C_{i}\left((a-2) C_{i}+D_{i}\right)+1 \\
& =(a-2) C_{i}^{2}+C_{i} D_{i}+1 \\
& =P_{i}+(a-2) C_{i}^{2}+2,
\end{aligned}
$$

where we used $P_{i}=C_{i} D_{i}-1$ in Lemma 1.11(2).

Claim 2. For any $i \geq 1$, it holds that

$$
B_{i}{ }^{2}-D_{i}{ }^{2}=(a-2) P_{i}
$$

Proof. In the case $i=1$, we can check it directly. By the inductive definitions of $B_{i}$ and $C_{i}$, we have

$$
\begin{aligned}
& B_{i+1}+D_{i+1}=(a-2) C_{i}+D_{i}+B_{i}, \\
& B_{i+1}-D_{i+1}=(a-2) C_{i}+D_{i}-B_{i} .
\end{aligned}
$$

Thus, under the assertion $B_{i}{ }^{2}-D_{i}{ }^{2}=(a-2) P_{i}$ of induction, we have

$$
\begin{aligned}
B_{i+1}^{2}-D_{i+1}^{2} & =\left(B_{i+1}+D_{i+1}\right)\left(B_{i+1}-D_{i+1}\right) \\
& =(a-2)^{2} C_{i}^{2}+2(a-2) C_{i} D_{i}+\left(D_{i}+B_{i}\right)\left(D_{i}-B_{i}\right) \\
& =(a-2)\left\{(a-2) C_{i}^{2}+2 C_{i} D_{i}-P_{i}\right\} \\
& =(a-2)\left\{(a-2) C_{i}^{2}+P_{i}+2\right\} \\
& =(a-2) P_{i+1} .
\end{aligned}
$$

where we used $C_{i} D_{i}=P_{i}+1$ in Lemma 1.11(2) and Claim 1. Lemma 1.11(3) follows from Claim 2.

We postpone the proof of Lemma 1.11(4) until Subsection 3.6.

\subsection{Extension to matrices and symmetry}

For the later use (in Section 4), we extend the sequences of the pairs $(A, B)$ and $(C, D)$ to those of matrices whose determinants are \pm 1 :

Definition 3.2 We define

$$
\left(\begin{array}{ll}
A_{0} & E_{0} \\
B_{0} & F_{0}
\end{array}\right)=\left(\begin{array}{cc}
-1 & 1 \\
1 & 0
\end{array}\right), \quad\left(\begin{array}{cc}
C_{0} & G_{0} \\
D_{0} & H_{0}
\end{array}\right)=\left(\begin{array}{ll}
1 & 0 \\
1 & 1
\end{array}\right)
$$

and

$$
\begin{aligned}
& \left(\begin{array}{ll}
A_{i+1} & E_{i+1} \\
B_{i+1} & F_{i+1}
\end{array}\right)=\left(\begin{array}{cc}
1 & 0 \\
a-2 & 1
\end{array}\right)\left(\begin{array}{ll}
C_{i} & G_{i} \\
D_{i} & H_{i}
\end{array}\right), \\
& \left(\begin{array}{ll}
C_{i+1} & G_{i+1} \\
D_{i+1} & H_{i+1}
\end{array}\right)=\left(\begin{array}{cc}
1 & a+2 \\
0 & 1
\end{array}\right)\left(\begin{array}{ll}
A_{i} & E_{i} \\
B_{i} & F_{i}
\end{array}\right),
\end{aligned}
$$


inductively. Thus we have

$$
\begin{aligned}
\left(\begin{array}{ll}
A_{1} & E_{1} \\
B_{1} & F_{1}
\end{array}\right)=\left(\begin{array}{cc}
1 & 0 \\
a-1 & 1
\end{array}\right), & \left(\begin{array}{ll}
C_{1} & G_{1} \\
D_{1} & H_{1}
\end{array}\right)=\left(\begin{array}{cc}
a+1 & 1 \\
1 & 0
\end{array}\right), \\
\left(\begin{array}{ll}
A_{2} & E_{2} \\
B_{2} & F_{2}
\end{array}\right)=\left(\begin{array}{cc}
a+1 & 1 \\
a^{2}-a-1 & a-2
\end{array}\right), & \left(\begin{array}{ll}
C_{2} & G_{2} \\
D_{2} & H_{2}
\end{array}\right)=\left(\begin{array}{cc}
a^{2}+a-1 & a+2 \\
a-1 & 1
\end{array}\right), \\
\left(\begin{array}{ll}
A_{3} & E_{3} \\
B_{3} & F_{3}
\end{array}\right)=\left(\begin{array}{cc}
a^{2}+a-1 & a+2 \\
a^{3}-a^{2}-2 a+1 & a^{2}-3
\end{array}\right), & \left(\begin{array}{ll}
C_{3} & G_{3} \\
D_{3} & H_{3}
\end{array}\right)=\left(\begin{array}{cc}
a^{3}+a^{2}-2 a-1 & a^{2}-3 \\
a^{2}-a-1 & a-2
\end{array}\right) .
\end{aligned}
$$

Lemma 3.3 $A_{i} F_{i}-B_{i} E_{i}=-(-1)^{i}, C_{i} H_{i}-D_{i} G_{i}=(-1)^{i}$.

\section{Lemma 3.4}

(1) For any $i \geq 0$, it holds that

$$
\left(\begin{array}{c}
A_{i} \\
B_{i}
\end{array}\right)=\left(\begin{array}{c}
E_{i+1} \\
F_{i+1}
\end{array}\right)-(-1)^{i}\left(\begin{array}{c}
E_{i} \\
F_{i}
\end{array}\right), \quad\left(\begin{array}{c}
C_{i} \\
D_{i}
\end{array}\right)=\left(\begin{array}{c}
G_{i+1} \\
H_{i+1}
\end{array}\right)+(-1)^{i}\left(\begin{array}{c}
G_{i} \\
H_{i}
\end{array}\right) .
$$

(2) For any $i \geq 1$, it holds that

$$
a\left(\begin{array}{c}
E_{i} \\
F_{i}
\end{array}\right)=\left(\begin{array}{c}
A_{i} \\
B_{i}
\end{array}\right)-(-1)^{i}\left(\begin{array}{c}
A_{i-1} \\
B_{i-1}
\end{array}\right), \quad a\left(\begin{array}{c}
G_{i} \\
H_{i}
\end{array}\right)=\left(\begin{array}{c}
C_{i} \\
D_{i}
\end{array}\right)+(-1)^{i}\left(\begin{array}{c}
C_{i-1} \\
D_{i-1}
\end{array}\right) .
$$

(3) For any $i \geq 0$, it holds that

$$
\left(\begin{array}{c}
B_{i} \\
F_{i}
\end{array}\right)=\left(\begin{array}{c}
a\left(D_{i}-(-1)^{i} H_{i}\right)+(-1)^{i} D_{i} \\
D_{i}-(-1)^{i} H_{i}
\end{array}\right), \quad\left(\begin{array}{c}
C_{i} \\
G_{i}
\end{array}\right)=\left(\begin{array}{c}
a\left(A_{i}+(-1)^{i} E_{i}\right)-(-1)^{i} A_{i} \\
A_{i}+(-1)^{i} E_{i}
\end{array}\right) .
$$

Proof. First we prove (1) and (2). In the initial cases, we can check them directly. The higher cases are proved by induction, using column decomposition of the inductive definition of the matrices in Definition 3.2.

Next we prove (3). In the case $i=0$, we can check them directly. Suppose $i \geq 1$. First, we show the second entry. The second equality in (1) includes $D_{i}-(-1)^{i} H_{i}=H_{i+1}$. We also have $H_{i+1}=F_{i}$ in Definition 3.2. Next, we show the first entry. The first equality in (2) includes $B_{i}-a F_{i}=(-1)^{i} B_{i-1}$. We also have $B_{i-1}=D_{i}$ in Definition 3.2. We have the first equality of (3). The second equality can be proved similarly.

Considering the values $\mathcal{X}(-a, i) \mathrm{s}$ for $\mathcal{X}=A, B, \cdots$ and $H$, we have a symmetry:

\section{Lemma 3.5}

$$
\begin{array}{ll}
A(-a, i)=-(-1)^{i} D(a, i), & E(-a, i)=(-1)^{i} H(a, i), \\
B(-a, i)=(-1)^{i} C(a, i), & F(-a, i)=-(-1)^{i} G(a, i) .
\end{array}
$$

Relating this lemma, on the 4-manifolds in Theorem 1.12, we have:

Corollary 3.6 The 4-manifold $X_{A B}(-a, i)$ is diffeomorphic to $X_{C D}(a, i)$ by an orientationreversing diffeomorphism, i.e.,

$$
X_{A B}(-a, i)=-X_{C D}(a, i) .
$$

Thus it is sufficient to prove the theorem only in the positive case $a \geq 0$. 


\subsection{Trivial cases}

There are some trivial cases in Theorem 1.12, i.e., the cases that both $T\left(A_{i}, B_{i}\right), T\left(C_{i}, D_{i}\right)$ are unknotted. In such cases, both pieces $X_{A B}$ and $X_{C D}$ are diffeomorphic to the total space of a disk bundle over $S^{2}$. It is well-known that a union of such manifolds is diffeomorphic to the required 4-manifold, see Kirby [K2, Lemma 4.4]. Here, we list such cases.

First, in the case $a=0$, the sequence of the matrices has period 4 . They are equal to

$$
\left(\begin{array}{ll}
A_{i} & C_{i} \\
B_{i} & D_{i}
\end{array}\right)=\left(\begin{array}{cc}
-1 & 1 \\
1 & 1
\end{array}\right),\left(\begin{array}{cc}
1 & 1 \\
-1 & 1
\end{array}\right),\left(\begin{array}{cc}
1 & -1 \\
-1 & -1
\end{array}\right),\left(\begin{array}{cc}
-1 & -1 \\
1 & -1
\end{array}\right)
$$

according to $i \equiv 0,1,2,3 \bmod 4$, respectively.

Next, in the case $a= \pm 1$, the sequence of the matrices (with respect to $i$ ) has period 6 (or 3). If $a=1$, the matrices are equal to

$$
\left(\begin{array}{cc}
-1 & 1 \\
1 & 1
\end{array}\right),\left(\begin{array}{ll}
1 & 2 \\
0 & 1
\end{array}\right),\left(\begin{array}{cc}
2 & 1 \\
-1 & 0
\end{array}\right),\left(\begin{array}{cc}
1 & -1 \\
-1 & -1
\end{array}\right),\left(\begin{array}{cc}
-1 & -2 \\
0 & -1
\end{array}\right),\left(\begin{array}{cc}
-2 & -1 \\
1 & 0
\end{array}\right)
$$

according to $i \equiv 0,1,2,3,4,5 \bmod 6$, respectively. If $a=-1$,

$$
\left(\begin{array}{cc}
-1 & 1 \\
1 & 1
\end{array}\right),\left(\begin{array}{cc}
1 & 0 \\
-2 & 1
\end{array}\right),\left(\begin{array}{cc}
0 & -1 \\
1 & -2
\end{array}\right)
$$

according to $i \equiv 0,1,2 \bmod 3$, respectively.

In the case $a=2$ or $a=-2$, it satisfies

$$
\left(\begin{array}{ll}
A_{i} & C_{i} \\
B_{i} & D_{i}
\end{array}\right)=\left(\begin{array}{cc}
2 i-1 & 2 i+1 \\
1 & 1
\end{array}\right) \text { or }(-1)^{i}\left(\begin{array}{cc}
-1 & 1 \\
2 i+1 & -(2 i-1)
\end{array}\right)
$$

respectively.

On the other hand, even if $|a| \geq 3$, the cases of $i=1$ are trivial.

$$
\left(\begin{array}{ll}
A_{1} & C_{1} \\
B_{1} & D_{1}
\end{array}\right)=\left(\begin{array}{cc}
1 & a+1 \\
a-1 & 1
\end{array}\right)
$$

This is the reason why we often assume that $a \geq 3$ and $i \geq 2$. In such cases, the following inequalities are easy to show.

Lemma 3.7 In the cases $a \geq 3$ and $i \geq 2$, we have

$$
\begin{aligned}
& C(a, i)>B(a, i)>A(a, i)>D(a, i)>1, \\
& B(a, i)>A(a, i)>F(a, i)>E(a, i)>0, \\
& C(a, i)>G(a, i)>D(a, i)>H(a, i)>0 .
\end{aligned}
$$

Finally, we study trivial cases in Theorem 1.18, for the other sequences. They are cases $i=0$ and one more pair $\left(\left(S_{1}, T_{1}\right),\left(U_{1}, V_{1}\right)\right)$ :

$$
\begin{aligned}
& \left(\begin{array}{ll}
S_{0} & U_{0} \\
T_{0} & V_{0}
\end{array}\right)=\left(\begin{array}{ll}
0 & 2 \\
1 & 1
\end{array}\right),\left(\begin{array}{ll}
s_{0} & u_{0} \\
t_{0} & v_{0}
\end{array}\right)=\left(\begin{array}{ll}
0 & 1 \\
1 & 2
\end{array}\right), \\
& \left(\begin{array}{ll}
K_{0} & M_{0} \\
L_{0} & N_{0}
\end{array}\right)=\left(\begin{array}{ll}
1 & 1 \\
3 & 3
\end{array}\right),\left(\begin{array}{ll}
k_{0} & m_{0} \\
l_{0} & n_{0}
\end{array}\right)=\left(\begin{array}{ll}
1 & 1 \\
2 & 2
\end{array}\right)
\end{aligned}
$$


and

$$
\left(\begin{array}{ll}
S_{1} & U_{1} \\
T_{1} & V_{1}
\end{array}\right)=\left(\begin{array}{ll}
1 & 1 \\
1 & 3
\end{array}\right)
$$

We also point out that the following four cases are the same pair of lens space surgeries:

$$
\left(\left(S_{2}, T_{2}\right),\left(U_{2}, V_{2}\right)\right),\left(\left(s_{1}, t_{1}\right),\left(u_{1}, v_{1}\right)\right),\left(\left(K_{1}, L_{1}\right),\left(M_{1}, N_{1}\right)\right), \quad\left(\left(k_{1}, l_{1}\right),\left(m_{1}, n_{1}\right)\right) .
$$

They are the pair $(U ; 5)=-L(5,1)$ and $(T(2,3) ; 5)=L(5,1)$, where $U$ is the unknot.

\subsection{Formulas on the second sequences}

First, we study the sequence $[S T U V]$. Definition 1.13 is reformulated as

$$
\left(\begin{array}{l}
S_{i+1} \\
T_{i+1} \\
U_{i+1} \\
V_{i+1}
\end{array}\right)=\left(\begin{array}{llll}
0 & 1 & 0 & 0 \\
1 & 0 & 0 & 1 \\
0 & 0 & 0 & 1 \\
0 & 1 & 1 & 0
\end{array}\right)\left(\begin{array}{c}
S_{i} \\
T_{i} \\
U_{i} \\
V_{i}
\end{array}\right)
$$

The $4 \times 4$ matrix has four eigenvalues $\sigma,-\sigma, \tau$ and $-\tau$, where

$$
\sigma=\frac{\sqrt{5}+1}{2}, \quad \tau=\frac{1}{\sigma}=\frac{\sqrt{5}-1}{2} .
$$

Thus, for $X=S, T, U$ or $V, X_{i}$ is a linear combination of $\sigma^{i},(-\sigma)^{i}, \tau^{i}$ and $(-\tau)^{i}$ :

$$
X_{i}=c_{1} \sigma^{i}+c_{2}(-\sigma)^{i}+c_{3} \tau^{i}+c_{4}(-\tau)^{i} .
$$

One can check that

$$
\begin{aligned}
& S_{i}=T_{i-1}=\frac{1}{\sqrt{5}}\left(\sigma^{i+1}+(-\sigma)^{i-1}-\tau^{i-1}-(-\tau)^{i+1}\right), \\
& U_{i}=V_{i-1}=\frac{1}{\sqrt{5}}\left(\sigma^{i+1}-(-\sigma)^{i-1}+\tau^{i-1}-(-\tau)^{i+1}\right) .
\end{aligned}
$$

Using relations

$$
\sigma \tau=1, \sigma^{2}=\sigma+1, \tau^{2}=-\tau+1, \sigma^{2}+1=\sqrt{5} \sigma, \tau^{2}+1=\sqrt{5} \tau
$$

and so on, we also have an explicit expression

$$
\begin{aligned}
& S_{2 j}=T_{2 j-1}=\frac{\sigma^{2 j}-\tau^{2 j}}{\sqrt{5}}, \quad U_{2 j}=V_{2 j-1}=\begin{array}{c}
\sigma^{2 j}+\tau^{2 j} \\
T_{2 j}=S_{2 j+1}=\sigma^{2 j+1}-\tau^{2 j+1},
\end{array} \quad V_{2 j}=U_{2 j+1}=\frac{\sigma^{2 j+1}+\tau^{2 j+1}}{\sqrt{5}} .
\end{aligned}
$$

Using relations (3.3) again, it is not hard to show the following lemma. We leave the details to the readers.

Lemma 3.8 ([ST] $)$ The pairs $\left(S_{i}, T_{i}\right)$ and $\left(U_{i}, V_{i}\right)$ satisfy the followings:

(1) $U_{i} T_{i-1}-V_{i} S_{i-1}=1, U_{i} T_{i+1}-V_{i} S_{i+1}=1$ and $S_{i} V_{i-1}-T_{i} U_{i-1}=-1$. 
(2) $S_{i} T_{i}+1=U_{i} V_{i}-1$. We call this integer $P_{i}$.

(3) If $i$ is even, then $S_{i}^{2}+V_{i}^{2}=P_{i}$ and $T_{i}^{2}+U_{i}^{2}=5 P_{i}$. Otherwise, $S_{i}^{2}+V_{i}^{2}=5 P_{i}$ and $T_{i}^{2}+U_{i}^{2}=P_{i}$.

(4) It holds the following recursive formulas:

$$
\left(\begin{array}{c}
S_{i+1} \\
T_{i+1}
\end{array}\right)=\left(\begin{array}{c}
U_{i} \\
V_{i}
\end{array}\right)+\left(\begin{array}{c}
S_{i-1} \\
T_{i-1}
\end{array}\right), \quad\left(\begin{array}{c}
U_{i+1} \\
V_{i+1}
\end{array}\right)=\left(\begin{array}{c}
S_{i} \\
T_{i}
\end{array}\right)+\left(\begin{array}{c}
U_{i-1} \\
V_{i-1}
\end{array}\right) .
$$

(5) $S_{i}=T_{i-1}, U_{i}=V_{i-1}$.

The following proposition follows from the inductive definition and Lemma 3.8(4).

Proposition 3.9 ([ST]) For even $i \geq 2$, we have $S_{i}<V_{i}<U_{i}<T_{i}$. For odd $i \geq 3$, we have $U_{i}<T_{i}<S_{i}<V_{i}$.

Next, we study the sequence $[s t u v]$. The method is similar to the sequence $[S T U V]$. Definition of the pairs $\left(s_{i}, t_{i}\right)$ and $\left(u_{i}, v_{i}\right)$ in Definition 1.13 is reformulated as

$$
\left(\begin{array}{l}
s_{i+1} \\
t_{i+1} \\
u_{i+1} \\
v_{i+1}
\end{array}\right)=\left(\begin{array}{llll}
0 & 1 & 0 & 0 \\
1 & 0 & 0 & 2 \\
0 & 0 & 0 & 1 \\
0 & 2 & 1 & 0
\end{array}\right)\left(\begin{array}{c}
s_{i} \\
t_{i} \\
u_{i} \\
v_{i}
\end{array}\right)
$$

The $4 \times 4$ matrix has four eigenvalues $\sigma^{\prime},-\sigma^{\prime}, \tau^{\prime}$ and $-\tau^{\prime}$, where

$$
\sigma^{\prime}=\sqrt{2}+1, \quad \tau^{\prime}=\sqrt{2}-1
$$

Thus, for $X=s, t, u$ or $v, X_{i}$ is a linear combination of $\sigma^{\prime i},\left(-\sigma^{\prime}\right)^{i}, \tau^{\prime i}$ and $\left(-\tau^{\prime}\right)^{i}$. We have an explicit expression

$$
\begin{aligned}
& s_{2 j}=t_{2 j-1}=\frac{{\sigma^{\prime 2 j}}^{2 j} \tau^{\prime 2 j}}{\sqrt{2}}, \quad u_{2 j}=v_{2 j-1}=\frac{{\sigma^{\prime 2 j}+\tau^{\prime 2 j}}_{2}}{2}, \quad v_{2 j}=u_{2 j+1}=\frac{\sigma^{\prime 2 j+1}+\tau^{\prime 2 j+1}}{\sqrt{2}} . \\
& t_{2 j}=s_{2 j+1}=\frac{\sigma^{\prime 2 j+1}-\tau^{\prime 2 j+1}}{2} .
\end{aligned}
$$

Using relations

$$
\sigma^{\prime} \tau^{\prime}=1, \sigma^{\prime 2}=2 \sigma^{\prime}+1, \delta^{2}=-2 \tau^{\prime}+1, \sigma^{\prime 2}+1=2 \sqrt{2} \sigma^{\prime}, \tau^{\prime 2}+1=2 \sqrt{2} \tau^{\prime}
$$

and so on, it is not hard to show the following lemma. We leave the details to the readers.

Lemma 3.10 The pairs $\left(s_{i}, t_{i}\right)$ and $\left(u_{i}, v_{i}\right)$ satisfy the followings:

(1) $u_{i} t_{i-1}-v_{i} s_{i-1}=2, u_{i} t_{i+1}-v_{i} s_{i+1}=2$ and $s_{i} v_{i-1}-t_{i} u_{i-1}=-2$.

(2) $s_{i} t_{i}+1=u_{i} v_{i}-1$. We call this integer $p_{i}$.

(3) If $i$ is even, then $s_{i}^{2}+v_{i}^{2}=4 p_{i}$ and $t_{i}^{2}+u_{i}^{2}=2 p_{i}$. Otherwise, $s_{i}^{2}+v_{i}^{2}=2 p_{i}$ and $t_{i}^{2}+u_{i}^{2}=4 p_{i}$. 
(4) It holds the following recursive formulas:

$$
\left(\begin{array}{c}
s_{i+1} \\
t_{i+1}
\end{array}\right)=2\left(\begin{array}{c}
u_{i} \\
v_{i}
\end{array}\right)+\left(\begin{array}{c}
s_{i-1} \\
t_{i-1}
\end{array}\right), \quad\left(\begin{array}{c}
u_{i+1} \\
v_{i+1}
\end{array}\right)=2\left(\begin{array}{c}
s_{i} \\
t_{i}
\end{array}\right)+\left(\begin{array}{c}
u_{i-1} \\
v_{i-1}
\end{array}\right) .
$$

(5) $s_{i}+t_{i} \equiv 1, u_{i}+v_{i} \equiv 1 \bmod 2$. Thus every $p_{i}$ is odd.

(6) $u_{i-1}+s_{i}, v_{i-1}+t_{i}\left(=u_{i}+s_{i+1}\right), s_{i-1}+u_{i}$ and $s_{i}+v_{i}\left(=t_{i-1}+u_{i+1}\right)$ are always even. We define their halves:

$$
\begin{array}{ll}
e_{i}=\left(u_{i-1}+s_{i}\right) / 2, & g_{i}=\left(s_{i-1}+u_{i}\right) / 2, \\
f_{i}=\left(v_{i-1}+t_{i}\right) / 2, & h_{i}=\left(s_{i}+v_{i}\right) / 2
\end{array}
$$

Then it holds that $s_{i} f_{i}-t_{i} e_{i}=-1, u_{i} h_{i}-v_{i} g_{i}=1$.

(7) $s_{i}=t_{i-1}, u_{i}=v_{i-1}$ and $f_{i}=e_{i-1}, h_{i}=g_{i-1}$.

The following proposition follows from the inductive definition and Lemma 3.10(4).

Proposition 3.11 For even $i \geq 1$, we have $u_{i}<s_{i}<t_{i}<v_{i}$. For odd $i \geq 1$, we have $s_{i}<u_{i}<v_{i}<t_{i}$.

Proof of Lemma 1.14(1)(2)(3). They follow from Lemma 3.8(1)(2)(3) for [STUV], and from Lemma 3.10(6)(2)(3) for [stuv], respectively.

\subsection{Formulas on the third sequences}

First, we study the sequence $[K L M N]$. We extend the sequences of pairs $\left(K_{i}, L_{i}\right)$ and $\left(M_{i}, N_{i}\right)$ in Definition 1.15 to those of matrices whose determinants are \pm 1 :

Definition $3.12 \quad$ We define

$$
\left(\begin{array}{ll}
K_{1} & E_{1} \\
L_{1} & F_{1}
\end{array}\right)=\left(\begin{array}{ll}
1 & 0 \\
6 & 1
\end{array}\right), \quad\left(\begin{array}{ll}
M_{1} & G_{1} \\
N_{1} & H_{1}
\end{array}\right)=\left(\begin{array}{ll}
2 & 1 \\
3 & 2
\end{array}\right)
$$

and

$$
\begin{aligned}
\left(\begin{array}{ll}
K_{i+1} & E_{i+1} \\
L_{i+1} & F_{i+1}
\end{array}\right) & =\left(\begin{array}{cc}
1 & 0 \\
9 & -1
\end{array}\right)\left(\begin{array}{cc}
M_{i} & G_{i} \\
N_{i} & H_{i}
\end{array}\right), \\
\left(\begin{array}{ll}
M_{i+1} & G_{i+1} \\
N_{i+1} & H_{i+1}
\end{array}\right) & =\left(\begin{array}{cc}
-1 & 1 \\
0 & 1
\end{array}\right)\left(\begin{array}{ll}
K_{i} & E_{i} \\
L_{i} & F_{i}
\end{array}\right)
\end{aligned}
$$

inductively.

Then we have:

Lemma 3.13 $K_{i} F_{i}-L_{i} E_{i}=-(-1)^{i}, M_{i} H_{i}-N_{i} G_{i}=-(-1)^{i}$.

Lemma 3.14 
(1) For any $i \geq 2$, it holds that

$$
3\left(\begin{array}{c}
E_{i} \\
F_{i}
\end{array}\right)=\left(\begin{array}{c}
K_{i} \\
L_{i}
\end{array}\right)+(-1)^{i}\left(\begin{array}{c}
K_{i-1} \\
L_{i-1}
\end{array}\right), \quad 3\left(\begin{array}{c}
G_{i} \\
H_{i}
\end{array}\right)=\left(\begin{array}{c}
M_{i} \\
N_{i}
\end{array}\right)-(-1)^{i}\left(\begin{array}{c}
M_{i-1} \\
N_{i-1}
\end{array}\right) .
$$

(2) For any $i \geq 1$, it holds that

$$
3 F_{i}=L_{i}+(-1)^{i} N_{i}, \quad 3 G_{i}=M_{i}-(-1)^{i} K_{i} .
$$

(3) For any $i \geq 1$, it holds that

$$
\left(\begin{array}{c}
E_{i+1} \\
F_{i+1}
\end{array}\right)+(-1)^{i}\left(\begin{array}{c}
E_{i} \\
F_{i}
\end{array}\right)=\left(\begin{array}{c}
K_{i} \\
L_{i}
\end{array}\right), \quad\left(\begin{array}{c}
G_{i+1} \\
H_{i+1}
\end{array}\right)-(-1)^{i}\left(\begin{array}{c}
G_{i} \\
H_{i}
\end{array}\right)=\left(\begin{array}{c}
M_{i} \\
N_{i}
\end{array}\right) .
$$

(4) For any $i \geq 1$, it holds that

$$
G_{i}+(-1)^{i} E_{i}=K_{i}, \quad F_{i}-(-1)^{i} H_{i}=N_{i} .
$$

Proof. (1) In the initial case, we can check it directly. The higher cases are proved by induction, using column decomposition of the inductive definition in Definition 3.12.

(2) The first (and the second, respectively) equality follows from the second (and the first) row of the equality (1) and $N_{i}=L_{i-1}$ (and $K_{i}=M_{i-1}$ ) in Definition 3.12.

(3), (4) By using the first equality in (1) twice,

$$
3\left(\begin{array}{c}
E_{i+1} \\
F_{i+1}
\end{array}\right)=\left(\begin{array}{c}
K_{i+1} \\
L_{i+1}
\end{array}\right)+(-1)^{i+1}\left(\begin{array}{c}
K_{i} \\
L_{i}
\end{array}\right), \quad 3\left(\begin{array}{c}
E_{i} \\
F_{i}
\end{array}\right)=\left(\begin{array}{c}
K_{i} \\
L_{i}
\end{array}\right)+(-1)^{i}\left(\begin{array}{c}
K_{i-1} \\
L_{i-1}
\end{array}\right),
$$

we have

$$
\left(\begin{array}{c}
E_{i+1} \\
F_{i+1}
\end{array}\right)+(-1)^{i}\left(\begin{array}{c}
E_{i} \\
F_{i}
\end{array}\right)=\frac{1}{3}\left\{\left(\begin{array}{c}
K_{i+1} \\
L_{i+1}
\end{array}\right)+\left(\begin{array}{c}
K_{i-1} \\
L_{i-1}
\end{array}\right)\right\}=\left(\begin{array}{c}
K_{i} \\
L_{i}
\end{array}\right) .
$$

By the first row of (3), since $E_{i+1}=G_{i}$, we have the first equality of (4). The second halves of (3) and (4) can be proved similarly by the second equality in (1).

Next, we study the sequence $[k l m n]$. We extend the sequences of pairs $\left(k_{i}, l_{i}\right)$ and $\left(m_{i}, n_{i}\right)$ in Definition 1.15 to those of matrices whose determinants are \pm 1 :

Definition 3.15 We define

$$
\left(\begin{array}{ll}
k_{1} & e_{1} \\
l_{1} & f_{1}
\end{array}\right)=\left(\begin{array}{ll}
1 & 0 \\
6 & 1
\end{array}\right), \quad\left(\begin{array}{ll}
m_{1} & g_{1} \\
n_{1} & h_{1}
\end{array}\right)=\left(\begin{array}{ll}
3 & 1 \\
2 & 1
\end{array}\right)
$$

and

$$
\begin{aligned}
\left(\begin{array}{ll}
k_{i+1} & e_{i+1} \\
l_{i+1} & f_{i+1}
\end{array}\right) & =\left(\begin{array}{cc}
1 & 0 \\
8 & -1
\end{array}\right)\left(\begin{array}{ll}
m_{i} & g_{i} \\
n_{i} & h_{i}
\end{array}\right), \\
\left(\begin{array}{ll}
m_{i+1} & g_{i+1} \\
n_{i+1} & h_{i+1}
\end{array}\right) & =\left(\begin{array}{cc}
-1 & 2 \\
0 & 1
\end{array}\right)\left(\begin{array}{ll}
k_{i} & e_{i} \\
l_{i} & f_{i}
\end{array}\right)
\end{aligned}
$$

inductively. 
Lemma 3.16 $k_{i} f_{i}-l_{i} e_{i}=-(-1)^{i}, m_{i} h_{i}-n_{i} g_{i}=-(-1)^{i}$.

\section{Lemma 3.17}

(1) For any $i \geq 2$, it holds that

$$
4\left(\begin{array}{c}
e_{i} \\
f_{i}
\end{array}\right)=\left(\begin{array}{c}
k_{i} \\
l_{i}
\end{array}\right)+(-1)^{i}\left(\begin{array}{c}
k_{i-1} \\
l_{i-1}
\end{array}\right), \quad 4\left(\begin{array}{c}
g_{i} \\
h_{i}
\end{array}\right)=\left(\begin{array}{c}
m_{i} \\
n_{i}
\end{array}\right)-(-1)^{i}\left(\begin{array}{c}
m_{i-1} \\
n_{i-1}
\end{array}\right) .
$$

(2) For any $i \geq 1$, it holds that

$$
4 f_{i}=l_{i}+(-1)^{i} n_{i}, \quad 4 g_{i}=m_{i}-(-1)^{i} k_{i} .
$$

(3) For any $i \geq 1$, it holds that

$$
\left(\begin{array}{c}
e_{i+1} \\
f_{i+1}
\end{array}\right)+(-1)^{i}\left(\begin{array}{c}
e_{i} \\
f_{i}
\end{array}\right)=\left(\begin{array}{c}
k_{i} \\
l_{i}
\end{array}\right), \quad\left(\begin{array}{l}
g_{i+1} \\
h_{i+1}
\end{array}\right)-(-1)^{i}\left(\begin{array}{c}
g_{i} \\
h_{i}
\end{array}\right)=\left(\begin{array}{c}
m_{i} \\
n_{i}
\end{array}\right) .
$$

(4) For any $i \geq 1$, it holds that

$$
g_{i}+(-1)^{i} e_{i}=k_{i}, \quad f_{i}-(-1)^{i} h_{i}=n_{i} .
$$

The proof is similar to that of Lemma 3.14, thus we omit it.

Finally, we study the explicit expression of the sequences $\left\{b_{i}\right\},\left\{d_{i}\right\}$ in Definition 1.16. We recall $\sigma, \tau$ from (3.2) and define $\kappa, \lambda$ by

$$
\sigma=\frac{\sqrt{5}+1}{2}, \tau=\frac{\sqrt{5}-1}{2}, \quad \kappa=\frac{\sqrt{3}+1}{\sqrt{2}}, \lambda=\frac{\sqrt{3}-1}{\sqrt{2}} .
$$

They satisfy $\sigma^{2}+\tau^{2}=3, \kappa^{2}+\lambda^{2}=4, \sigma \tau=\kappa \lambda=1$ and

$$
\sigma^{2}-1=\sigma, \tau^{2}-1=-\tau, \quad \kappa^{2}-1=\sqrt{2} \kappa, \lambda^{2}-1=-\sqrt{2} \lambda .
$$

It is easy to see

$$
\begin{aligned}
& b_{i}=\frac{\left(\sigma^{2 i}-\tau^{2 i}\right)-\left(\sigma^{2(i-1)}-\tau^{2(i-1)}\right)}{\sigma^{2}-\tau^{2}}=\frac{\sigma^{2 i-1}+\tau^{2 i-1}}{\sqrt{5}}, \\
& d_{i}=\frac{\left(\kappa^{2 i}-\lambda^{2 i}\right)-\left(\kappa^{2(i-1)}-\lambda^{2(i-1)}\right)}{\kappa^{2}-\lambda^{2}}=\frac{\kappa^{2 i-1}+\lambda^{2 i-1}}{\sqrt{6}} .
\end{aligned}
$$

Using these expressions, we have:

\section{Lemma 3.18}

(1) $K_{i}^{2}+M_{i}^{2}=P_{i}$ and $L_{i}^{2}+N_{i}^{2}=9 P_{i}$.

(2) $k_{i}{ }^{2}+m_{i}{ }^{2}=2 p_{i}$ and $l_{i}{ }^{2}+n_{i}{ }^{2}=8 p_{i}$.

Proof of Lemma 1.17(1)(2)(3). Lemma 1.17(1) follows from Lemma 3.13 and Lemma 3.16. Lemma 1.17(2) is easier than the other sequences. Lemma 1.17(3) follows from Lemma 3.18. 


\subsection{Proof of the properties (4) in Lemmas}

Let $[X y z \mathcal{W}]$ be one of the sequences $[A B C D],[S T U V],[s t u v],[K L M N]$ and $[k l m n]$. Then, Lemma 1.11(4), Lemma 1.14(4) and Lemma 1.17(4) are commonly rewritten as

$$
X_{i}^{4} \not \equiv \pm 1 \quad \bmod P_{i} .
$$

Note that $P_{i}\left(=x_{i} y_{i}-1\right.$ or $\left.X_{i} y_{i}+1\right)$ depends on the choice of sequences. A congruence $X_{i}^{4} \equiv \pm 1$ is equivalent to $X_{i}^{2} \mp y_{i}^{2} \equiv 0$, since $\bar{X}_{i}{ }^{2} \equiv y_{i}{ }^{2}$. We focus on the ratios $X_{i} / y_{i}$ and its inverse $y_{i} / X_{i}$ and their limits, since

$$
\lim _{i \rightarrow \infty} \frac{x_{i}}{y_{i}}=\lim _{i \rightarrow \infty} \frac{x_{i}^{2}}{x_{i} y_{i}}=\lim _{i \rightarrow \infty} \frac{x_{i}^{2}}{P_{i}}, \quad \lim _{i \rightarrow \infty} \frac{y_{i}}{x_{i}}=\lim _{i \rightarrow \infty} \frac{y_{i}{ }^{2}}{x_{i} y_{i}}=\lim _{i \rightarrow \infty} \frac{y_{i}{ }^{2}}{P_{i}}
$$

We also study

$$
\lim _{i \rightarrow \infty}\left(\frac{x_{i}}{y_{i}}+\frac{y_{i}}{x_{i}}\right)=\lim _{i \rightarrow \infty} \frac{x_{i}{ }^{2}+y_{i}{ }^{2}}{x_{i} y_{i}}=\lim _{i \rightarrow \infty} \frac{x_{i}{ }^{2}+y_{i}{ }^{2}}{P_{i}} .
$$

We start with the first sequence $[A B C D]$. The limits of the ratio depends on the index $a$. Proof of Lemma 1.11(4). Suppose $A_{i}{ }^{4} \equiv 1 \bmod P_{i}$. It implies $A_{i}{ }^{2} \equiv{\overline{A_{i}}}^{2} \equiv B_{i}{ }^{2} \equiv{D_{i}}^{2}$ $\bmod P_{i}$, where $\overline{A_{i}}$ is the inverse of $A_{i}$ in $\left(\mathbb{Z} / P_{i} \mathbb{Z}\right)^{*}$. Here, we used Lemma 1.11(3) in the third equivalence. It contradicts $1<D_{i}{ }^{2}<A_{i}{ }^{2}<P_{i}$, see Lemma 3.7.

Next, suppose $A_{i}{ }^{4} \equiv-1 \bmod P_{i}$. It implies $A_{i}{ }^{2}+D_{i}{ }^{2} \equiv 0 \bmod P_{i}$ by the similar argument as above. We prove that it implies a contradiction by estimation of the ratios $A_{i} / B_{i}$ and $C_{i} / D_{i}$ (they increase and converge), but the proof is a little harder, divided into two cases. We omit the detail of the estimation. In the case $a \geq 4,2 A_{i}<B_{i}$ and $2 D_{i}<C_{i}$. Since $P_{i}=A_{i} B_{i}+1=C_{i} D_{i}-1$, they imply $2 A_{i}^{2}<P_{i}$ and $2 D_{i}^{2}<P_{i}$, thus $A_{i}{ }^{2}+D_{i}{ }^{2}<P_{i}$. We have a contradiction. On the other hand, in the case $a=3$, we can estimate $6 A_{i}>5 B_{i}$ except the case $i=2$, and $6 D_{i}>C_{i}$. They implies $6 A_{i}^{2}>5 P_{i}$ and $6 D_{i}^{2}>P_{i}$, thus $2 P_{i}>A_{i}{ }^{2}+D_{i}{ }^{2}>P_{i}$. We have a contradiction. The proof is complete.

For the other sequences, the limits of the ratios $X_{i} / y_{i}$ (or $x_{2 i} / y_{2 i}, x_{2 i-1} / y_{2 i-1}$ for $[S T U V]$ and $[s t u v])$ are non-zero and irrational numbers, see Table 4 . Thus the required non-congruences

\begin{tabular}{|c|l|l|l|}
\hline Sequence & $X_{2 i} / y_{2 i}$ & $y_{2 i} / X_{2 i}$ & $\left(X_{2 i}{ }^{2}+y_{2 i}{ }^{2}\right) / P_{2 i}$ \\
\hline$\left(S_{i}, T_{i}\right)$ & $\frac{5-\sqrt{5}}{10}=0.27639 \ldots$ & $3.61803 \ldots$ & $\frac{15+2 \sqrt{5}}{5}=3.89442 \ldots$ \\
\hline$\left(s_{i}, t_{i}\right)$ & $2-\sqrt{2}=0.58578 \ldots$ & $1.70710 \ldots$ & $\frac{6-\sqrt{2}}{2}=2.29289 \ldots$ \\
\hline \hline Sequence & $X_{i} / y_{i}$ & $y_{i} / X_{i}$ & $\left(X_{i}{ }^{2}+y_{i}{ }^{2}\right) / P_{i}$ \\
\hline$\left(K_{i}, L_{i}\right)$ & $\frac{3-\sqrt{5}}{6}=0.12732 \ldots$ & $7.85410 \ldots$ & $\frac{15+4 \sqrt{5}}{3}=7.98142 \ldots$ \\
\hline$\left(k_{i}, l_{i}\right)$ & $\frac{2-\sqrt{3}}{2}=0.13397 \ldots$ & $7.46410 \ldots$ & $\frac{10+3 \sqrt{3}}{2}=7.59807 \ldots$ \\
\hline
\end{tabular}

Table 4: Limits of ratios

hold for sufficiently large $i$. For a complete proof for all $i$, we use some evaluations. To evaluate $x_{i} / y_{i}$, we mainly use $x_{I} / y_{I}$ and $y_{I} / X_{I}$ for some $I$. 
Proof of Lemma 1.14(4) for $[S T U V]$. Note that

$$
\lim _{j \rightarrow \infty} \frac{S_{2 j}^{2}}{P_{2 j}}=\lim _{j \rightarrow \infty} \frac{S_{2 j}}{T_{2 j}}=\lim _{j \rightarrow \infty} \frac{U_{2 j-1}^{2}}{P_{2 j-1}}=\lim _{j \rightarrow \infty} \frac{U_{2 j-1}}{V_{2 j-1}}=\frac{1}{\sqrt{5} \sigma}=\frac{5-\sqrt{5}}{10},
$$

and

$$
\frac{3}{11}<\frac{5-\sqrt{5}}{10}=0.276393 \ldots<\frac{2}{7} .
$$

Claim 1. For any integer $j \geq 3$, it holds that

$$
\frac{3}{11}<\frac{S_{2 j}}{T_{2 j}}<\frac{2}{7} \text { and } \quad \frac{3}{11}<\frac{U_{2 j-1}}{V_{2 j-1}}<\frac{2}{7} .
$$

This claim is proved by Lemma 3.8(4) and induction.

Claim 2. For $j \geq 3$, we have the following inequalities:

$$
\begin{array}{ll}
S_{2 j}{ }^{2}<\frac{2}{7} P_{2 j}, & \frac{7}{2} P_{2 j}<T_{2 j}{ }^{2}<\frac{11}{3} P_{2 j}, \\
U_{2 j-1}{ }^{2}<\frac{2}{7} P_{2 j-1}, & \frac{7}{2} P_{2 j-1}<V_{2 j-1}{ }^{2}<\frac{11}{3} P_{2 j-1} .
\end{array}
$$

Proof. By Claim 1, we have $7 S_{2 j}<2 T_{2 j}$ and $3 T_{2 j}<11 S_{2 j}$. Thus

$$
7 S_{2 j}{ }^{2}<2 S_{2 j} T_{2 j}<2\left(S_{2 j} T_{2 j}+1\right)=2 P_{2 j}, \quad 3 T_{2 j}{ }^{2}<11 S_{2 j} T_{2 j}<11\left(S_{2 j} T_{2 j}+1\right)=11 P_{2 j} .
$$

We also have $2 V_{2 j-1}>7 U_{2 j}$, thus

$$
2 V_{2 j-1}^{2}>7 U_{2 j-1} V_{2 j-1}>7\left(U_{2 j-1} V_{2 j-1}-1\right)=7 P_{2 j-1} .
$$

The other inequalities are a little troublesome. By Claim 1, we have $2 T_{2 j}>7 S_{2 j}$, thus $2 T_{2 j}^{2}>7 S_{2 j} T_{2 j}$. Here, since the difference $2 T_{2 j}-7 S_{2 j}$ is an integer and $T_{2 j}$ is sufficiently large, we can show $2 T_{2 j}{ }^{2}>7 S_{2 j} T_{2 j}+7=7 P_{2 j}$. We can show the other inequalities similarly.

Claim 3. For $j \geq 3$, we have the following inequalities:

$$
\begin{aligned}
& (-, \text { ev }) \frac{S_{2 j}{ }^{2}}{P_{2 j}}<\frac{2}{7}, \frac{7}{2}<\frac{T_{2 j}{ }^{2}}{P_{2 j}}-1<\frac{2}{3}, \quad(-, \text { od }) \frac{U_{2 j-1}{ }^{2}}{P_{2 j-1}}<\frac{2}{7}, \frac{7}{2}<\frac{V_{2 j-1}{ }^{2}}{P_{2 j-1}}-3<\frac{2}{3} \\
& (+, \text { ev }) \frac{7}{2} P_{2 j}<S_{2 j}{ }^{2}+T_{2 j}{ }^{2}<\frac{83}{21} P_{2 j}, \quad(+, \text { od }) \quad \frac{7}{2} P_{2 j-1}<U_{2 j-1}{ }^{2}+V_{2 j-1}{ }^{2}<\frac{83}{21} P_{2 j-1} \text {. }
\end{aligned}
$$

These inequalities are proved by Claim 2 .

We go back to the proof of Lemma 1.14(4). The cases $i=3,4$ can be shown directly. Suppose $i \geq 5$ and $S_{i}{ }^{4} \equiv 1 \bmod P_{i}$. It implies both $S_{i}{ }^{2} \equiv T_{i}{ }^{2} \bmod P_{i}$ and $U_{i}{ }^{2} \equiv V_{i}{ }^{2}$ $\bmod P_{i}$. In the case where $i$ is even $(i=2 j)$ (and $i$ is odd $(i=2 j-1)$, respectively), the first congruence (and the second congruence) contradicts $(-, \mathrm{ev})$ (and $(-, \mathrm{od}))$ in Claim 3.

Next, suppose $i \geq 5$ and $S_{i}{ }^{4} \equiv-1 \bmod P_{i}$. It implies both $S_{i}{ }^{2}+T_{i}{ }^{2} \equiv 0 \bmod P_{i}$ and $U_{i}{ }^{2}+V_{i}^{2} \equiv 0 \bmod P_{i}$. In the case $i$ is even (and $i$ is odd, respectively), the first congruence (and the second congruence) contradicts $(+, \mathrm{ev})($ and $(+, \mathrm{od}))$ in Claim 3. 
Proof of Lemma 1.14(4) for $[s t u v]$. The proof is similar to the case of $[S T U V]$, except the final step. We use

$$
2-\sqrt{2}=0.5878 \ldots<\frac{10}{17} .
$$

Claim 4. For any integer $j \geq 2$, it holds that

$$
\frac{s_{2 j}}{t_{2 j}}<\frac{10}{17} \text { and } \quad \frac{u_{2 j+1}}{v_{2 j+1}}<\frac{10}{17} .
$$

This claim is proved by Lemma 3.10(4) and induction. By applying the same method for the case $[S T U V]$ (we omit "Claim 2"), we can show the following claim:

Claim 5. For $j \geq 3$, we have the following inequalities:

$$
\begin{aligned}
& (-, \text { ev }) \frac{s_{2 j}{ }^{2}}{p_{2 j}}<\frac{10}{17}, \frac{7}{10}<\frac{t_{2 j}{ }^{2}}{p_{2 j}}-1<1, \quad(-, \text { od }) \frac{u_{2 j-1}{ }^{2}}{p_{2 j-1}}<\frac{10}{17}, \frac{7}{10}<\frac{v_{2 j-1}{ }^{2}}{p_{2 j-1}}-1<1 \\
& (+, \text { ev }) \frac{17}{10} p_{2 j}<s_{2 j}{ }^{2}+t_{2 j}{ }^{2}<\frac{44}{17} p_{2 j}, \quad(+, \text { od }) \quad \frac{17}{10} p_{2 j-1}<u_{2 j-1}{ }^{2}+v_{2 j-1}{ }^{2}<\frac{44}{17} p_{2 j-1} .
\end{aligned}
$$

We go back to the proof of Lemma 1.14(4). The cases $i=2,3$ can be shown directly. Suppose $i \geq 5$ and $s_{i}{ }^{4} \equiv 1 \bmod p_{i}$. It implies both $s_{i}{ }^{2} \equiv t_{i}{ }^{2} \bmod p_{i}$ and $u_{i}{ }^{2} \equiv v_{i}{ }^{2} \bmod p_{i}$. In the case $i$ is even (and $i$ is odd, respectively), the first congruence (and the second congruence) contradicts $(-$, ev) (and $(-$, od $))$ in Claim 5.

Next, suppose $i \geq 5$ and $s_{i}{ }^{4} \equiv-1 \bmod p_{i}$. It implies both $s_{i}{ }^{2}+t_{i}{ }^{2} \equiv 0 \bmod p_{i}$ and $u_{i}^{2}+v_{i}^{2} \equiv 0 \bmod p_{i}$. But, in the case where $i$ is even $(i=2 j), s_{2 j}{ }^{2}+t_{2 j}{ }^{2}=2 p_{i}$ (by $(+$, ev) in Claim 5) contradicts $s_{i}+t_{i} \equiv 1 \bmod 2$ in Lemma 3.10(1). In the case $i$ is odd, we have also a contradiction similarly. The proof of Lemma 1.14 is completed.

Proof of Lemma 1.17(4) for $[K L M N]$ and $[k l m n]$. The proof is similar to the case of [stuv]. We use an inequality

Claim 6. For any integer $i \geq 3$, it holds that

$$
\frac{3-\sqrt{5}}{6}<\frac{2-\sqrt{3}}{2}<\frac{1}{6}
$$

$$
\frac{K_{i}}{L_{i}}, \frac{k_{i}}{l_{i}}<\frac{1}{6} \text { and } \quad \frac{22}{3}<\frac{L_{i}}{K_{i}}, \frac{l_{i}}{k_{i}}<8 .
$$

This claim is proved by Definition 1.16. By the method similar to the previous cases, we can show:

Claim 7. For $i \geq 3$, we have the following inequalities:

$$
\begin{aligned}
& \text { (-) } \frac{K_{i}^{2}}{P_{i}}<\frac{1}{6}, \frac{1}{3}<\frac{L_{i}^{2}}{P_{i}}-7<1, \quad \frac{k_{i}^{2}}{p_{i}}<\frac{1}{6}, \frac{1}{3}<\frac{l_{i}^{2}}{p_{i}}-7<1 \\
& \text { (+) } \frac{22}{3} P_{i}<K_{i}{ }^{2}+L_{i}^{2}<\frac{49}{6} P_{i}, \quad \frac{22}{3} p_{i}<k_{i}{ }^{2}+l_{i}{ }^{2}<\frac{49}{6} p_{i} .
\end{aligned}
$$

We go back to the proof of Lemma 1.14(4). The cases $i=2$ can be shown directly. Suppose $i \geq 3$ and $K_{i}^{4} \equiv 1 \bmod p_{i}$. It implies $K_{i}{ }^{2} \equiv L_{i}{ }^{2} \bmod P_{i}$, which contradicts (-) in Claim 7 . Next, suppose $i \geq 3$ and $K_{i}{ }^{4} \equiv-1 \bmod P_{i}$. It implies $K_{i}{ }^{2}+L_{i}{ }^{2} \equiv 0 \bmod P_{i}$. But, by $(+)$ in Claim 7, $K_{i}{ }^{2}+L_{i}{ }^{2}=8 P_{i}$ contradicts $L_{i}{ }^{2}+N_{i}{ }^{2}=9 P_{i}$ in Lemma 3.18, since $N_{i}{ }^{2}-K_{i}{ }^{2}=P_{i}$ implies $8 b_{i}{ }^{2}=3 b_{i} b_{i+1}-1$ thus $b_{i}=1$. Here we used $b_{i}$ in Subsection 3.5. The proof for the case $[k l m n]$ can be done in the way similar to $[K L M N]$. The proof of Lemma 1.17 is completed. 


\section{3-dim calculus}

For the pairs of lens space surgeries defined in Section 1, we search both dual knots in the common resulting lens spaces by 3 -dim calculus, and describe the constructed 4-manifolds $X_{x y} \cup$ $\left(\mp X_{z \mathcal{W}}\right)$ by 4-dim diagrams. All diagrams are framed links of type $\left(T\left(\left(p_{z}, q_{z}\right),\left(p_{w}, q_{w}\right)\right) ; r_{z}, r_{w}\right)$ defined in Definition 2.1, and the parameters depend on the choice of the sequence $[X Y \mathcal{W} \mathcal{W}]=$ $\left(\left(\mathcal{X}_{i}, y_{i}\right),\left(z_{i}, \mathcal{W}_{i}\right)\right)$. The goal of this section is the following theorem:

\section{Theorem 4.1}

(1) The 4-manifold $X_{A B} \cup\left(-X_{C D}\right)$ is described by the framed link

$$
\left(T\left(\left(A_{i}, B_{i}\right),\left(A_{i-1}, B_{i-1}\right)\right) ; P_{i}, P_{i-1}\right) .
$$

(2) The 4-manifolds $X_{S T} \cup X_{U V}, X_{s t} \cup X_{u v}$ are described by the framed links

$$
\left(T\left(\left(S_{i}, T_{i}\right),\left(U_{i-1}, V_{i-1}\right)\right) ; P_{i}, P_{i-1}\right), \quad\left(T\left(\left(s_{i}, t_{i}\right),\left(u_{i-1}, v_{i-1}\right)\right) ; p_{i}, p_{i-1}\right),
$$

respectively.

(3) The 4-manifolds $X_{K L} \cup X_{M N}$ and $X_{k l} \cup X_{m n}$ are described by the framed links

$$
\left(T\left(\left(K_{i}, L_{i}\right),\left(K_{i-1}, L_{i-1}\right)\right) ; P_{i}, P_{i-1}\right), \quad\left(T\left(\left(k_{i}, l_{i}\right),\left(k_{i-1}, l_{i-1}\right)\right) ; p_{i}, p_{i-1}\right),
$$

respectively.

In other words, in each framed link, the second component is the attaching circle of the dualized 2-handle of the second piece. Here, $P_{i}, p_{i}\left(=Z_{i} \mathcal{W}_{i}-1\right)$ depend on the choice of the sequence.

See an example $X_{A B} \cup\left(-X_{C D}\right)$ with $i=2$ in Figure 26 .

\subsection{For the first sequence}

We assume $a \geq 3$ and $i \geq 2$. In Subsection 2.3, we reviewed Moser's lens space surgeries (Lemma 1.3) along any positive torus knots $T(p, q)$ and defined a convenient diagram $\mathcal{D}(p, q)$ in Lemma 2.4. Almost all diagrams are divided into two cases according to whether $i$ is even or odd. We often synthesize them by using a notation $\varepsilon_{i}=(-1)^{i}$. We start with the long diagram $\mathcal{D}\left(A_{i}, B_{i}\right)$ :

Lemma 4.2 For the pair $\left(A_{i}, B_{i}\right)$, the diagram $\mathcal{D}\left(A_{i}, B_{i}\right)$ is as in Figure 13. In each diagram, the notation $[\cdots]^{j-1}$ means $(j-1)$ times iteration of the block $[\cdots]$ (The horizontal line is cut at the terminal vertices).

Proof. We can verify the theorem in the case $i=2,3$ directly. The higher $i$ cases are shown by induction as below. In the first steps in Algorithm (1), the words $w\left(A_{i}, B_{i}\right), w\left(C_{i}, D_{i}\right)$ satisfy

$$
\begin{aligned}
& \left(A_{i+1}, B_{i+1}\right)=\left(C_{i},(a-2) C_{i}+D_{i}\right) \rightarrow_{R^{a-2}}\left(C_{i}, D_{i}\right) \rightarrow_{L} \cdots, \\
& \left(C_{i+1}, D_{i+1}\right)=\left(A_{i}+(a+2) B_{i}, B_{i}\right) \rightarrow_{L^{a+2}}\left(A_{i}, B_{i}\right) \rightarrow_{R} \cdots .
\end{aligned}
$$




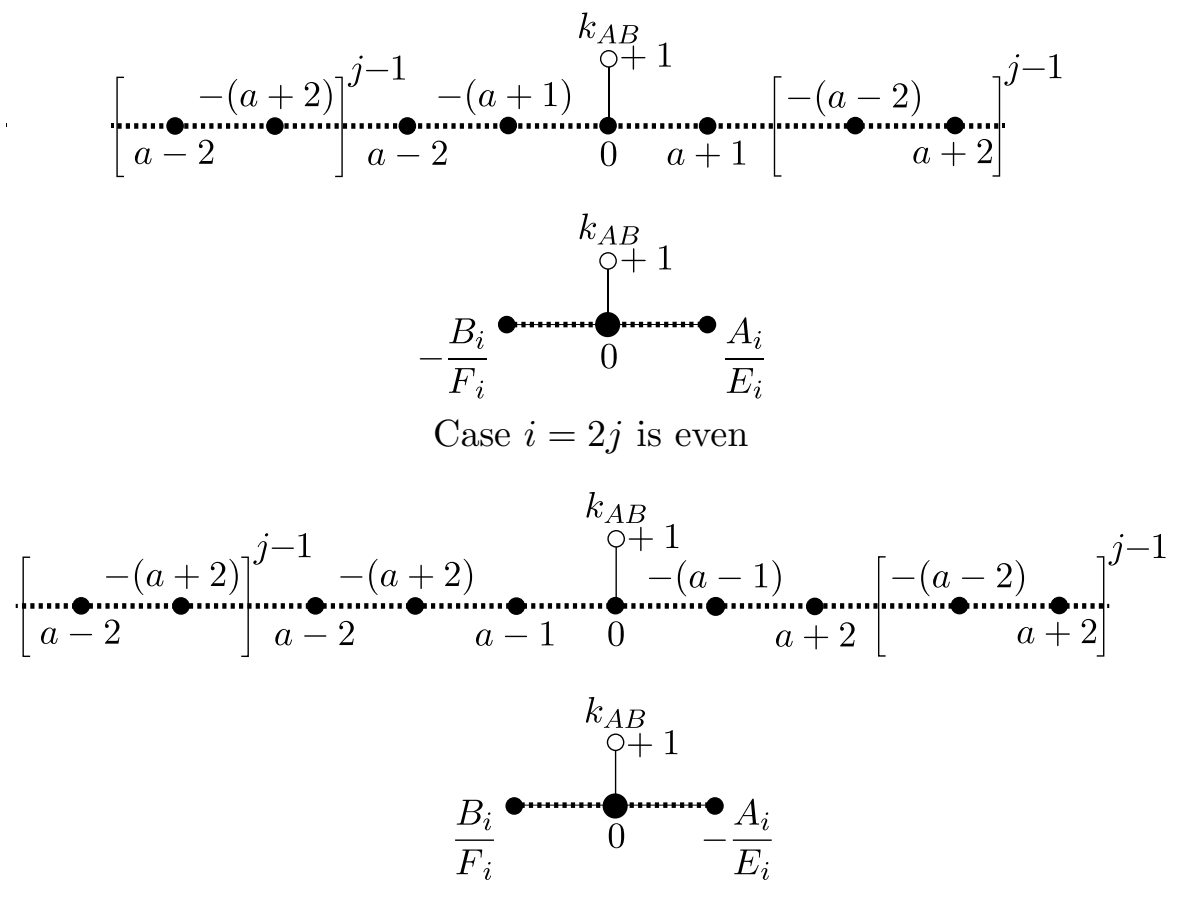

Case $i=2 j+1$ is odd

Figure 13: Diagram $\mathcal{D}\left(A_{i}, B_{i}\right)$, where $A_{i} F_{i}-B_{i} E_{i}=-(-1)^{i}$

Thus we have

$$
\begin{aligned}
& w\left(A_{i+2}, B_{i+2}\right)=R^{a-2} L^{a+2} w\left(A_{i}, B_{i}\right), \\
& w\left(C_{i+2}, D_{i+2}\right)=L^{a+2} R^{a-2} w\left(C_{i}, D_{i}\right) .
\end{aligned}
$$

These relations contribute as that the case $i+2$ is obtained from the case $i$ by adding one more copy of the block to both hand-sides.

To shorten the diagram $\mathcal{D}\left(A_{i}, B_{i}\right)$, we use the parameters $E_{i}, F_{i}$ satisfying $A_{i} F_{i}-B_{i} E_{i}=$ $-\varepsilon_{i}$, where $\varepsilon_{i}=(-1)^{i}$, defined in Subsection 3.2, see also Lemma 3.7.

The diagram $\mathcal{D}\left(C_{i}, D_{i}\right)$ is obtained by the same method, using $G_{i}, H_{i}$ satisfying $C_{i} H_{i}-$ $D_{i} G_{i}=\varepsilon_{i}$, see the top picture of Figure 14. Here we find a strange symmetry over the sequence $[A B C D]$, which we call $J$-symmetry:

Definition 4.3 (Operation $J$ ) For chain framed links whose coefficients are parametrized by an integer a (ex., $\mathcal{D}\left(A_{i}, B_{i}\right), \mathcal{D}\left(C_{i}, D_{i}\right)$ and related ones), we define an operation $J$ as

changing the sign of a (from \pm a to $\mp a$ ), and after that, take the mirror image.

Lemma 4.4 The operation $J$ interchanges $\mathcal{D}\left(A_{i}, B_{i}\right)$ and $\mathcal{D}\left(C_{i}, D_{i}\right)$, and also interchanges the knots $\left(k_{A B} ;+1\right)$ and $\left(k_{C D} ;-1\right)$. Thus $J$ keeps the diagram of the common lens space of the lens space surgeries. 

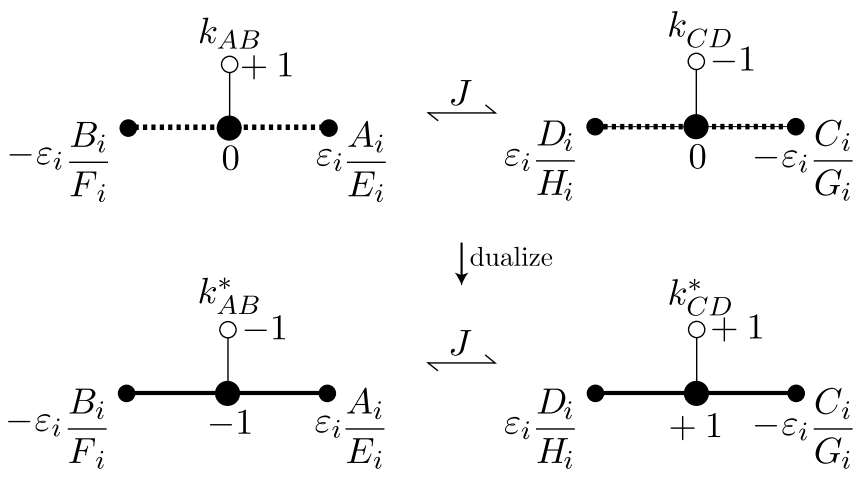

$\downarrow$ dualize
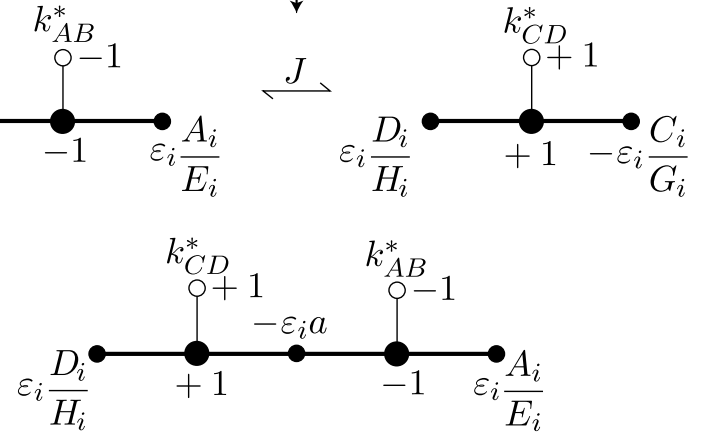

Figure 14: Diagram $\mathcal{L}(i)$ of the common lens space, where $\varepsilon_{i}=(-1)^{i}$

This lemma is proved by the relations in Lemma $3.5\left(B(-a, i)=\varepsilon_{i} C(a, i), F(-a, i)=\right.$ $-\varepsilon_{i} G(a, i)$ and so on $)$. Dualizing $\mathcal{D}\left(A_{i}, B_{i}\right) \cup\left(k_{A B} ;+1\right)$ and $\mathcal{D}\left(C_{i}, D_{i}\right) \cup\left(k_{C D} ;-1\right)$ respectively by Lemma 2.5 , we have a chain framed link of the common resulting lens space, see the middle picture of Figure 14. Caring $J$-symmetry, we modify the diagram by basic formulas and amalgamate them to search both dual knots in the common lens space. Here, we used Lemma 3.4(3) in the form

$$
\left[-\varepsilon_{i} a,+1, \varepsilon_{i} \frac{D_{i}}{H_{i}}\right]=-\varepsilon_{i}\left[a,-\varepsilon_{i},-\frac{D_{i}}{H_{i}}\right], \quad\left[-\varepsilon_{i} a,-1, \varepsilon_{i} \frac{A_{i}}{E_{i}}\right]=-\varepsilon_{i}\left[a, \varepsilon_{i},-\frac{A_{i}}{E_{i}}\right]
$$

and

$$
\begin{gathered}
{\left[a,-\varepsilon_{i},-\frac{D_{i}}{H_{i}}\right]=a-\frac{1}{-\varepsilon_{i}+\frac{H_{i}}{D_{i}}}=\frac{a\left(D_{i}-\varepsilon_{i} H_{i}\right)+\varepsilon_{i} D_{i}}{D_{i}-\varepsilon_{i} H_{i}}=\frac{B_{i}}{F_{i}},} \\
{\left[a, \varepsilon_{i}, \frac{A_{i}}{E_{i}}\right]=a-\frac{1}{\varepsilon_{i}+\frac{E_{i}}{A_{i}}}=\frac{a\left(A_{i}+\varepsilon_{i} E_{i}\right)-\varepsilon_{i} A_{i}}{A_{i}+\varepsilon_{i} E_{i}}=\frac{C_{i}}{G_{i}} .}
\end{gathered}
$$

By the results in Subsection 2.3, we summarize the arguments above as follows:

Lemma 4.5 See the 3-dim diagram in the bottom picture in Figure 14.

(1) The diagram $\mathcal{L}(i)$ presents the common lens space of both lens space surgeries

$$
M\left(T\left(A_{i}, B_{i}\right) ; A_{i} B_{i}+1\right) \cong M\left(T\left(C_{i}, D_{i}\right) ; C_{i} D_{i}-1\right) \cong-L\left(P_{i}, B_{i}{ }^{2}\right) .
$$

(2) In the diagram $\mathcal{L}(i)$ of the lens space, $(-1)$-framed $k_{A B}^{*}\left(\right.$ and $(+1)$-framed $k_{C D}^{*}$, respectively) is the dual knot of the lens space surgery along $T\left(A_{i}, B_{i}\right)$ (and along $T\left(C_{i}, D_{i}\right)$ ). 
(3) Operation $J$ keeps the diagram $\mathcal{L}(i)$ itself, and interchanges the dual knots $\left(k_{A B}^{*} ;-1\right)$ and $\left(k_{C D}^{*} ;+1\right)$ in $\mathcal{L}(i)$.

Proof (of Theorem 4.1(1)). We dualize $k_{A B}^{*}$ by Definition 2.6, see the top picture in Figure 15. We have a non-trivial diagram $\mathcal{D}\left(A_{i}, B_{i}\right)$ of $S^{3}$. By the duality, the $((+1)$-framed $)$ component $k_{A B}$ becomes $P_{i}$-framed $T\left(A_{i}, B_{i}\right)$ in the resulting $S^{3}$. In the complement, we have the other dual knot $k_{C D}^{*}$. We chase the link $k_{A B} \cup k_{C D}^{*}$ under the 3 -dim calculus from $\mathcal{D}\left(A_{i}, B_{i}\right)$ to the empty diagram. By Lemma $2.7(2)$ and (3), the resulting link is of type $T\left(\left(p_{z}, q_{z}\right),\left(p_{w}, q_{w}\right)\right)$ with $\left(p_{z}, q_{z}\right)=\left(A_{i}, B_{i}\right)$ in $S^{3}$, where we took $T\left(A_{i}, B_{i}\right)$ in the torus $T_{z}$. The final task is

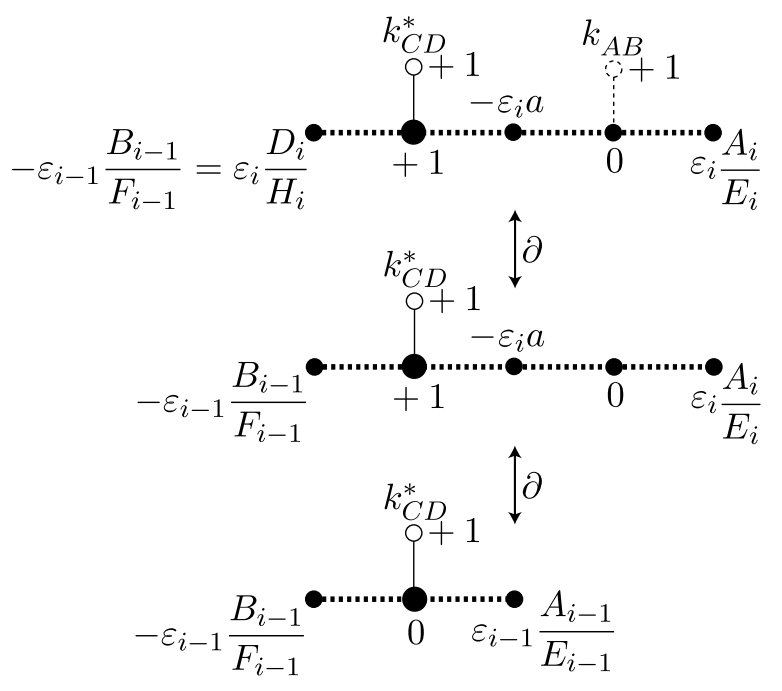

Figure 15: Check that $k_{C D}^{*}$ is $T_{w}\left(B_{i-1}, A_{i-1}\right)$.

to verify $\left(p_{w}, q_{w}\right)=\left(A_{i-1}, B_{i-1}\right)$. By 3 -dim calculus (ignoring $\left.k_{A B}\right)$ as in Figure 15 and an equality

$$
\left[1,-\varepsilon_{i} a, 0, \varepsilon_{i} \frac{A_{i}}{E_{i}}\right]=\left[1,-\varepsilon_{i}\left(a-\frac{A_{i}}{E_{i}}\right)\right]=\left[1, \frac{A_{i-1}}{E_{i}}\right]=\left[0, \frac{A_{i-1}}{E_{i}-A_{i-1}}\right]=\left[0, \varepsilon_{i-1} \frac{A_{i-1}}{E_{i-1}}\right],
$$

where we used Lemma 3.4(2) and (1), we can deform the diagram to $\mathcal{D}\left(A_{i-1}, B_{i-1}\right)$. Thus the component $k_{C D}^{*}$ is a torus knot $T_{w}\left(B_{i-1}, A_{i-1}\right)$ in the torus $T_{w}$.

\subsection{For the second and the third sequences}

For the second and the third sequences, the method is similar to the last subsection except taking a mirror image at the beginning.

Proof (of Theorem 4.1(2)). First, we study the case $[S T U V]$. We take a "mirror image" of the lens space surgery $M\left(T\left(U_{i}, V_{i}\right) ; U_{i} V_{i}-1\right)=L\left(P_{i}, T_{i}^{2}\right)$ :

$$
-M\left(T\left(U_{i}, V_{i}\right) ; U_{i} V_{i}-1\right)=M\left(T\left(U_{i}, V_{i}\right) ! ;-\left(U_{i} V_{i}-1\right)\right)=-L\left(P_{i}, T_{i}^{2}\right),
$$

where "mirror image" means a mirror image of the diagram of a surgery description of a lens space. We study the pair 


$$
M\left(T\left(U_{i}, V_{i}\right) ! ;-\left(U_{i} V_{i}-1\right)\right)=-L\left(P_{i}, T_{i}^{2}\right) \text { and } M\left(T\left(S_{i}, T_{i}\right) ;\left(S_{i} T_{i}+1\right)\right)=-L\left(P_{i}, T_{i}^{2}\right) .
$$

Modifying and amalgamating two diagrams of lens spaces as in Figure 16, we get both dual knots in a diagram of the common lens space. Here we used Lemma 3.8(3) and (4) as

$$
\left[-1, \frac{S_{i}}{U_{i-1}}\right]=-\frac{V_{i}}{T_{i-1}}, \quad\left[-1, \frac{U_{i}}{S_{i-1}}\right]=-\frac{T_{i}}{V_{i-1}} .
$$

Dualizing the dual knot $k_{S T}^{*}$ of the lens space surgery $M\left(T\left(S_{i}, T_{i}\right) ;\left(S_{i} T_{i}+1\right)\right)$, we have the
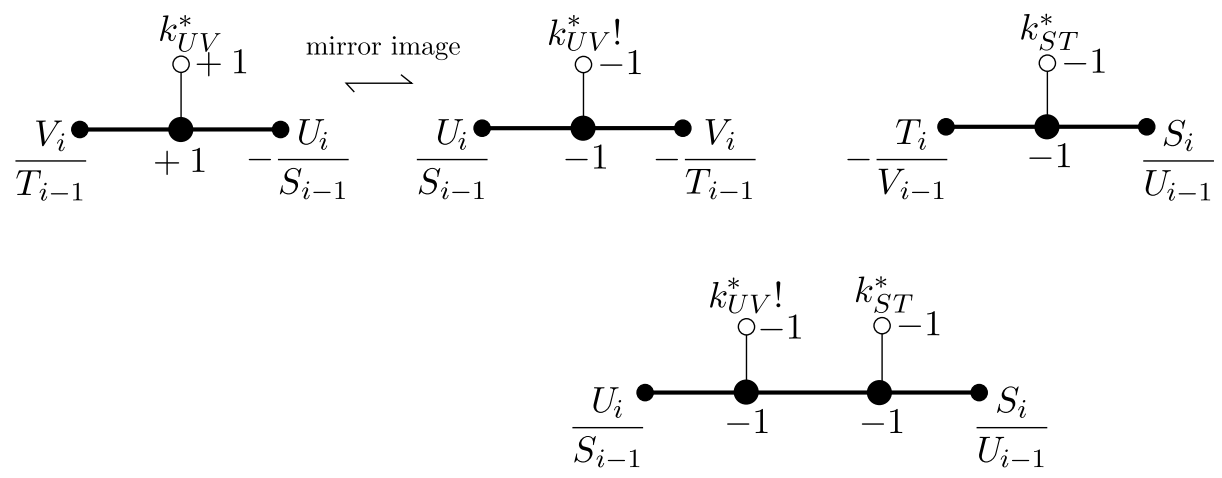

Figure 16: Diagram of the lens space (Case $[S T U V]$ )

first diagram in Figure 17. It is a non-trivial diagram of $S^{3}$ containing the required link. By

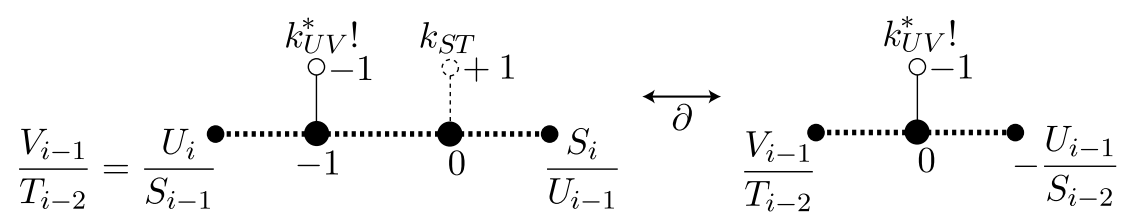

Figure 17: Check that $k_{U V}^{*}$ ! is $T_{w}\left(V_{i-1}, U_{i-1}\right)$

3-dim calculus (ignoring $k_{S T}$ ) in Figure 17, using an equality

$$
\left[-1,0, \frac{S_{i}}{U_{i-1}}\right]=\frac{S_{i}-U_{i-1}}{U_{i-1}}=\frac{S_{i-2}}{U_{i-1}}=\left[0,-\frac{U_{i-1}}{S_{i-2}}\right]
$$

by Lemma 3.8(4) and (5), we can deform the diagram to $\mathcal{D}\left(U_{i-1}, V_{i-1}\right)$. Thus the component $k_{U V}^{*}$ ! is a torus knot $T_{w}\left(U_{i-1}, V_{i-1}\right)$ in the torus $T_{w}$.

Second, we study the case $[s t u v]$. Since the method is completely same as the case $[S T U V]$, we only draw diagrams and equalities. We leave the detail to the reader. In Figure 18, we use

$$
\left[-2,-1, \frac{u_{i}}{g_{i}}\right]=-\frac{2 g_{i}+u_{i}}{g_{i}+u_{i}}, \quad\left[-2,-1, \frac{s_{i}}{e_{i}}\right]=-\frac{2 e_{i}+s_{i}}{e_{i}+s_{i}}
$$

and

$$
\begin{array}{ll}
2 g_{i}+u_{i}=s_{i-1}+2 u_{i}=s_{i+1}=t_{i}, & 2 e_{i}+s_{i}=u_{i-1}+2 s_{i}=u_{i+1}=v_{i} \\
2\left(g_{i}+u_{i}\right)=s_{i+1}+u_{i}=2 f_{i}, & 2\left(e_{i}+s_{i}\right)=u_{i+1}+s_{i}=2 h_{i}
\end{array}
$$



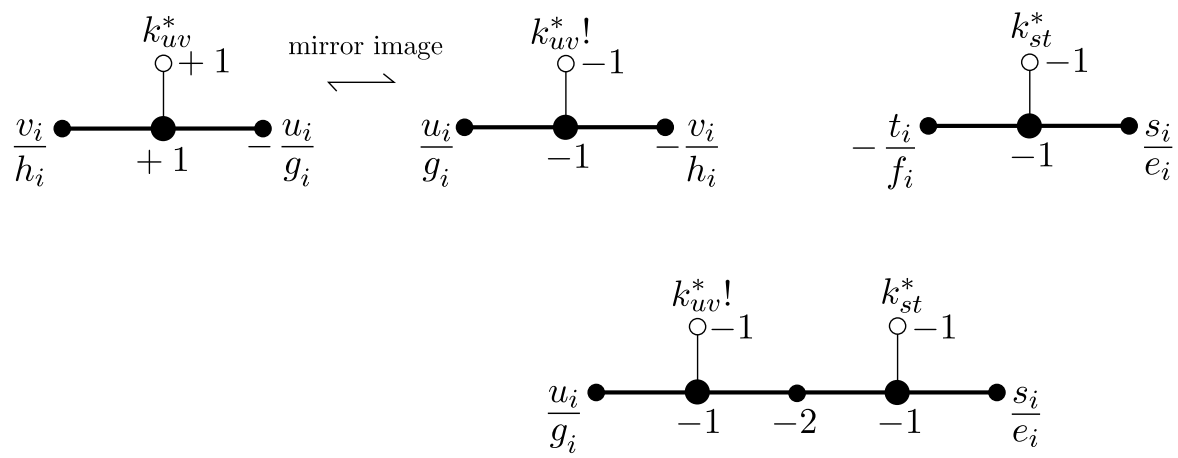

Figure 18: Diagram of the lens space (Case $[$ stuv])
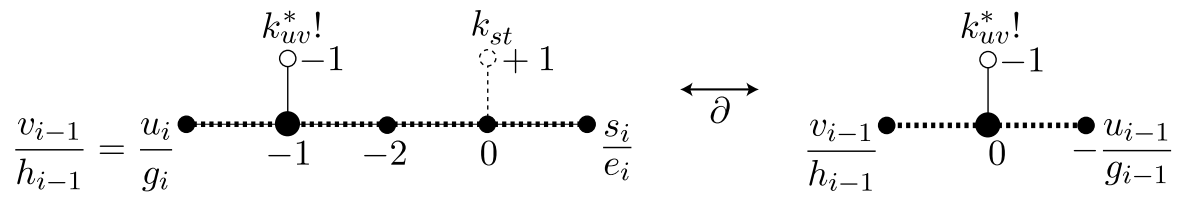

Figure 19: Check that $k_{u v}^{*}$ ! is $T_{w}\left(v_{i-1}, u_{i-1}\right)$

where we used Lemma 3.10(4) and (6). In Figure 19, we use

$$
\begin{aligned}
{\left[-1,-2,0, \frac{s_{i}}{e_{i}}\right] } & =\left[-1,-2+\frac{s_{i}}{e_{i}}\right]=\left[-1, \frac{s_{i}-2 e_{i}}{e_{i}}\right]=\left[-1,-\frac{u_{i-1}}{e_{i}}\right] \\
& =\frac{e_{i}-u_{i-1}}{u_{i-1}}=\left[0,-\frac{u_{i-1}}{e_{i}-u_{i-1}}\right]
\end{aligned}
$$

and $e_{i}-u_{i-1}=g_{i-1}$. The last equality is shown by

$$
2\left(e_{i}-g_{i-1}\right)=\left(u_{i-1}+s_{i}\right)-\left(s_{i-2}+u_{i-1}\right)=s_{i}-s_{i-2}=2 u_{i-1} .
$$

Next, we study the case of the third sequences. The method is similar.

Proof (of Theorem 4.1(3)). First, we study the case $[K L M N]$. We study the pair

$M\left(T\left(M_{i}, N_{i}\right) ! ;-\left(M_{i} N_{i}-1\right)\right)=-L\left(P_{i}, K_{i}^{2}\right)$ and $M\left(T\left(K_{i}, L_{i}\right) ;\left(K_{i} L_{i}+1\right)\right)=-L\left(P_{i}, K_{i}^{2}\right)$.

Modifying and amalgamating two diagrams of lens spaces in Figure 20, we get both dual knots in a diagram of the common lens space. Here we use Lemma 3.14(4) and (2) as

$$
\begin{gathered}
{\left[-3 \varepsilon_{i}, 1, \varepsilon_{i} \frac{K_{i}}{E_{i}}\right]=\left[-3 \varepsilon_{i}, 1-\varepsilon_{i} \frac{E_{i}}{K_{i}}\right]=\left[-3 \varepsilon_{i}, \frac{G_{i}}{K_{i}}\right]=-\varepsilon_{i}\left[3,-\varepsilon_{i} \frac{G_{i}}{K_{i}}\right],} \\
{\left[-3 \varepsilon_{i},-1, \varepsilon_{i} \frac{N_{i}}{H_{i}}\right]=\left[-3 \varepsilon_{i},-1-\varepsilon_{i} \frac{H_{i}}{N_{i}}\right]=\left[-3 \varepsilon_{i},-\frac{F_{i}}{N_{i}}\right]=-\varepsilon_{i}\left[3, \varepsilon_{i} \frac{F_{i}}{N_{i}}\right]}
\end{gathered}
$$



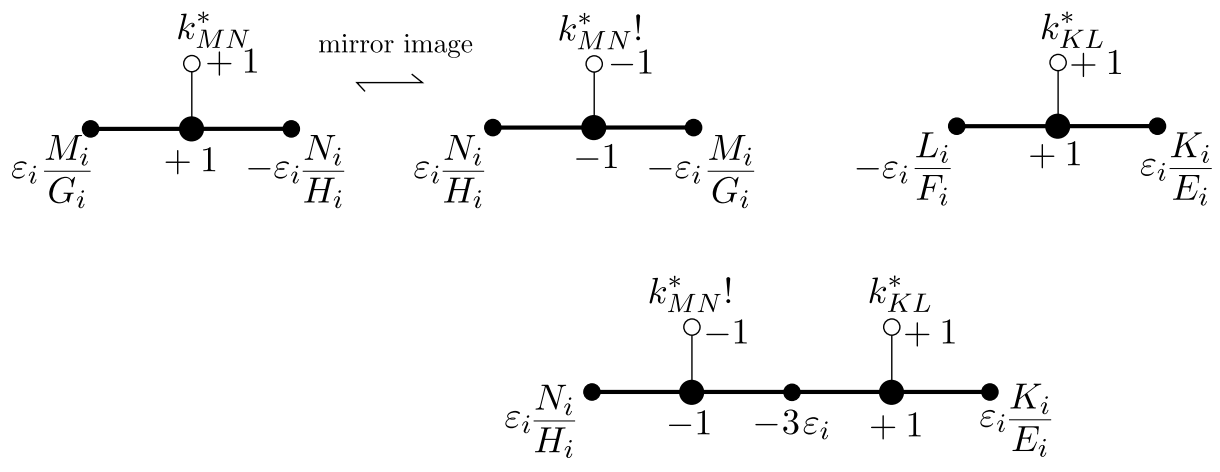

Figure 20: Diagram of the lens space (Case $[K L M N]$ )

and

$$
\left[3,-\varepsilon_{i} \frac{G_{i}}{K_{i}}\right]=\frac{3 G_{i}+\varepsilon_{i} K_{i}}{G_{i}}=\frac{M_{i}}{G_{i}}, \quad\left[3, \varepsilon_{i} \frac{F_{i}}{N_{i}}\right]=\frac{3 F_{i}-\varepsilon_{i} N_{i}}{F_{i}}=\frac{L_{i}}{F_{i}}
$$

Dualizing the dual knot $k_{K L}^{*}$, we have the first diagram in Figure 21. It is a non-trivial diagram of $S^{3}$ containing the required link. By 3-dim calculus (ignoring $k_{K L}$ ) in Figure 21

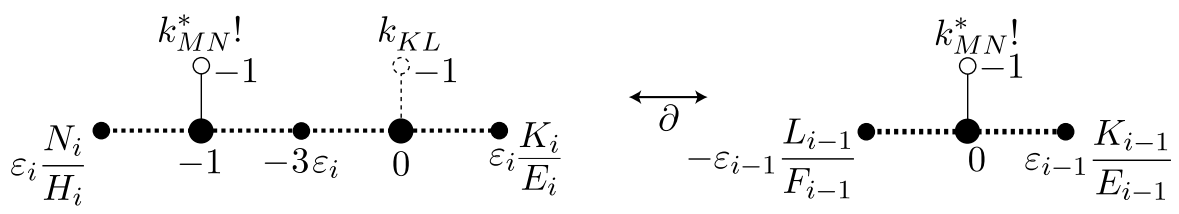

Figure 21: Check that $k_{M N}^{*} !$ is $T_{w}\left(L_{i-1}, K_{i-1}\right)$

using an equality

$$
\begin{aligned}
{\left[-1,-3 \varepsilon_{i}, 0, \varepsilon_{i} \frac{K_{i}}{E_{i}}\right] } & =\left[-1, \varepsilon_{i}\left(\frac{K_{i}-3 E_{i}}{E_{i}}\right)\right]=\left[-1,-\frac{K_{i-1}}{G_{i-1}}\right] \\
& =\frac{G_{i-1}-K_{i-1}}{K_{i-1}}=-\frac{\varepsilon_{i-1} E_{i-1}}{K_{i-1}}=\left[0, \varepsilon_{i-1} \frac{K_{i-1}}{E_{i-1}}\right]
\end{aligned}
$$

by Lemma 3.14(1),(5),(4) and Definition 3.12, we can deform the diagram to $\mathcal{D}\left(K_{i-1}, L_{i-1}\right)$. Thus the component $k_{M N}^{*}$ ! is a torus knot $T_{w}\left(L_{i-1}, K_{i-1}\right)$ in the torus $T_{w}$.

Finally, we study the case $[k l m n]$. Outline of the proof is the same as $[K L M N]$. We must replace $K, L, . ., H,-3 \varepsilon_{i}$ by $k, l, . ., h,-4 \varepsilon_{i}$ and use Lemma 3.17 instead of Lemma 3.14. We leave the details to the readers.

Here we point out that a recursive formula of words in Algorithm in Subsection 2.3. For $i \geq 2$,

$$
\begin{aligned}
w\left(S_{i+2}, T_{i+2}\right) & =w\left(S_{i}, T_{i}\right) L R, & & w\left(s_{i+2}, t_{i+2}\right)=w\left(s_{i}, t_{i}\right) L^{2} R^{2} \\
w\left(U_{i+2}, V_{i+2}\right) & =w\left(U_{i}, V_{i}\right) R L, & & w\left(u_{i+2}, v_{i+2}\right)=w\left(u_{i}, v_{i}\right) R^{2} L^{2} .
\end{aligned}
$$




$$
\begin{gathered}
w\left(K_{i+1}, L_{i+1}\right)=\left\{\begin{array}{ll}
w\left(K_{i}, L_{i}\right) R^{3} & (i \text { is even }) \\
w\left(K_{i}, L_{i}\right) R^{2} L & (i \text { i odd })
\end{array}, \quad w\left(k_{i+1}, l_{i+1}\right)=\left\{\begin{array}{ll}
w\left(k_{i}, l_{i}\right) R^{4} & (i \text { is even }), \\
w\left(k_{i}, l_{i}\right) R^{2} L^{2} & (i \text { is odd })
\end{array},\right.\right. \\
w\left(M_{i+1}, N_{i+1}\right)=\left\{\begin{array}{ll}
w\left(M_{i}, N_{i}\right) L^{2} R & (i \text { is even }) \\
w\left(M_{i}, N_{i}\right) L^{3} & (i \text { is odd })
\end{array}, \quad w\left(m_{i+1}, n_{i+1}\right)=\left\{\begin{array}{ll}
w\left(m_{i}, n_{i}\right) L^{2} R^{2} & (i \text { is even }) \\
w\left(m_{i}, n_{i}\right) L^{4} & (i \text { is odd })
\end{array} .\right.\right.
\end{gathered}
$$

They are contrast to the words (4.1) of $w\left(A_{i}, B_{i}\right)$ and $w\left(C_{i}, D_{i}\right)$.

\section{4-dim calculus}

\subsection{Key lemma: Handle-slides in a thickened torus}

We study framed links and handle-slides in a thickened torus $T^{2} \times I$, and apply it to 4-dim calculus in $S^{3}$, where we regard $T^{2}$ as $\mathbb{R}^{2} / \mathbb{Z}^{2}$ and $I$ an open interval in $\mathbb{R}$. Every manifold is regarded as an oriented manifold. For a coprime pair $(p, q)$ of integers, by $l(p, q)$, we denote a simple closed curve in $T^{2}$ derived from the line $\{(p t, q t+\epsilon) \mid t \in \mathbb{R}\} \subset \mathbb{R}^{2}$. By $l(p, q)[\tau]$ with $\tau \in I$, we mean a knot in $T^{2} \times I$ :

$$
l(p, q)[\tau]:=l(p, q) \times\{\tau\} \subset T^{2} \times\{\tau\} \subset T^{2} \times I .
$$

A framing (an isotopy class of a parallel shift) of $l(p, q)[\tau]$ is called 0 -framing or a torus framing if the parallel shift is taken in $T^{2} \times\{\tau\}$, and is called $r$-framing if it is right-handed $r$ full twists from 0 -framing. For a framed link, orientation of the components are not needed, but we take one choice here.

From now on, we fix $I=(-1.5,1.5)$. We are concerned with a framed link $\left(K_{0} ; r_{0}\right) \cup(K, r)$ in $T^{2} \times I$ with

$$
(K ; r)=(l(p, q)[-1] ; r), \quad\left(K_{0} ; r_{0}\right)=\left(l\left(p_{0}, q_{0}\right)[0] ; \pm 1\right) .
$$

We slide the handle $(K ; r)$ over $\left(K_{0} ; r_{0}\right)$. The assumption $r_{0}= \pm 1$ is important. On the handle-slides of the component $K$ over $K_{0}$, we have:

Lemma 5.1 Let $\left(K_{0} ; \delta\right) \cup(K ; r)$ be the framed link in $T^{2} \times I$ as above $(\delta=+1$ or -1$)$. We set $\Delta:=p_{0} q-q_{0} p$. There exists a sequence of handle-slides of $K$ over $K_{0}$ whose result is $\left(K_{0} ; \delta\right) \cup\left(K^{\prime} ; r^{\prime}\right)$ with

$$
\left(K^{\prime} ; r^{\prime}\right)=\left(l\left(p-\delta \Delta p_{0}, q-\delta \Delta q_{0}\right)[+1] ; r\right) .
$$

Note that $|\Delta|$ is the geometric intersection number of the curves $l\left(p_{0}, q_{0}\right)$ and $l(p, q)$ in the torus, and that, if $\delta=+1$ (or -1 , respectively) $l\left(p-\delta \Delta p_{0}, q-\delta \Delta q_{0}\right)$ is obtained by a righthanded (or a left-handed) Dehn twist along $l\left(p_{0}, q_{0}\right)$ from $l(p, q)$. Note that, even if we change the orientation of the component $l\left(p_{0}, q_{0}\right)\left(\right.$ to $\left.l\left(-p_{0},-q_{0}\right)\right)$, the result of the Dehn twist does not change, because $\Delta=p_{0} q-q_{0} p$ also changes the sign.

Proof. We only prove the lemma in the case $\delta=+1$. Otherwise, we take a mirror image. See the handle-slides in Figure 22, where each square denotes the fundamental domain of $T^{2}$. First, we fix $\left(p_{0}, q_{0}\right)=( \pm 1,0)$. They are in the cases of $(p, q)=(1,1),(1,-1)$ and $(2,3)$, respectively. The other cases are similar: If $p_{0} q>0$ (or $p_{0} q<0$, respectively), we slide the 
handle as in the $(1,1)$ case (or as $(1,-1)$ case) $|q|$ times at each intersection point. General $\left(p_{0}, q_{0}\right)$ cases are proved from them by the self diffeomorphism on $T^{2}$ defined by the matrix

$$
\left(\begin{array}{cc}
p_{0} & r_{0} \\
q_{0} & s_{0}
\end{array}\right) \quad \in S L(2 ; \mathbb{Z})
$$
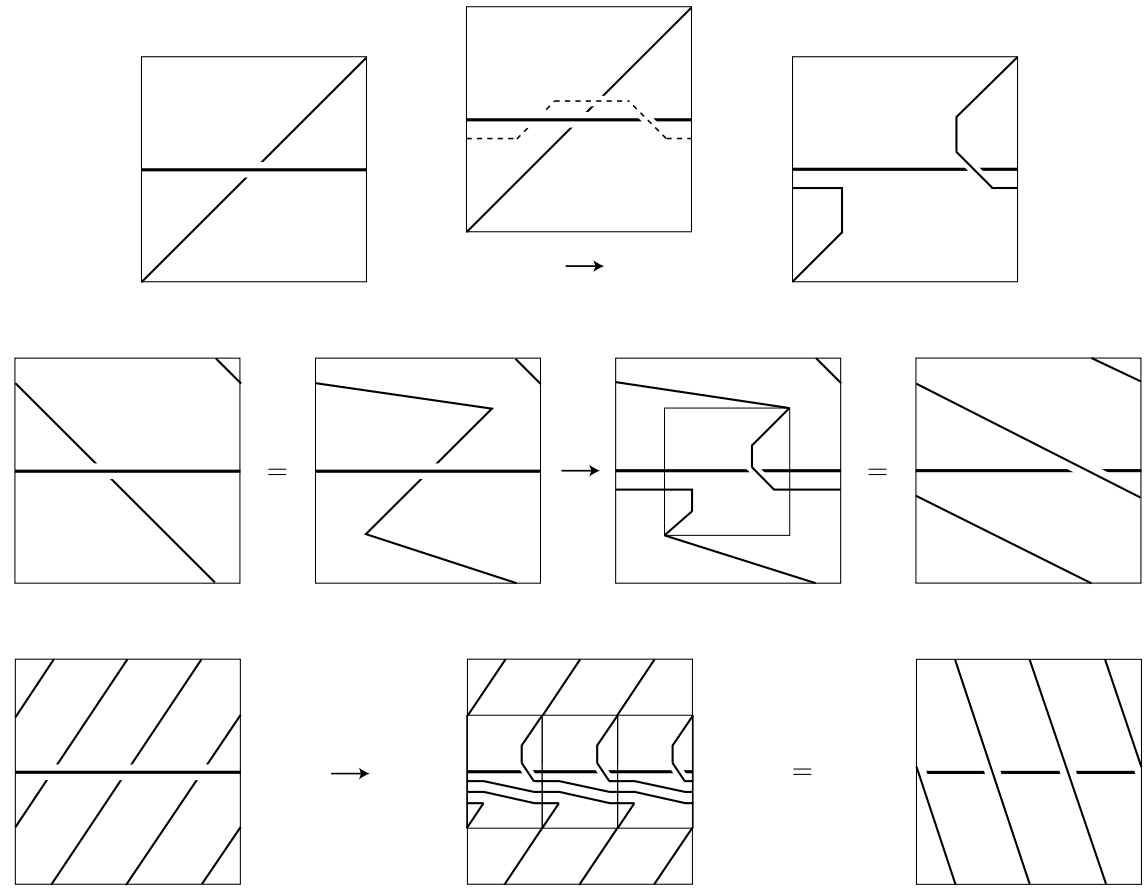

Figure 22: Handle-slides in $T^{2} \times I$

We regard this handle-slide as "When $K$ pass through the level $t=0, K_{0}$ acts on $K$ as a Dehn twist".

\subsection{4-dim calculus for each sequence}

We "map" the handle-slides in Lemma 5.1 into $S^{3}$. There exists an embedding $\Psi$ of $T^{2} \times I$ into $S^{3}$ that maps the following subsets homeomorphically.

$$
\begin{array}{lll}
\Psi\left(T^{2}[-1]\right)=T_{z}, & \Psi(l(1,0)[-1])=m_{z}, & \Psi(l(0,1)[-1])=l_{z}, \\
\Psi\left(T^{2}[0]\right)=T_{w}, & \Psi(l(1,0)[0])=l_{w}, & \Psi(l(0,1)[0])=m_{w},
\end{array}
$$

see Definition 2.1 for the notations of tori $T_{z}, T_{w}$ and so on. Note that $l(p, q)$ with the torus framing corresponds to the torus knot $T(p, q)$ with $p q$-framing.

Lemma 5.2 Let $\delta=+1$ or -1 . We study the framed link

$$
\left(T\left(\left(p_{z}, q_{z}\right),\left(p_{w}, q_{w}\right)\right) ; R_{z}, r_{w}\right)=\left(T_{z}\left(p_{z}, q_{z}\right) ; R_{z}\right) \cup\left(T_{w}\left(q_{w}, p_{w}\right) ; r_{w}\right),
$$


with $R_{z}=p_{z} q_{z}+r$ and $r_{w}=p_{w} q_{w}+\delta$. We set $\Delta:=p_{z} q_{w}-p_{w} q_{z}$. Then, there exists a sequence of handle-slides $T_{z}\left(p_{z}, q_{z}\right)$ over $T_{w}\left(q_{w}, p_{w}\right)$ whose result is

$$
\left(T\left(\left(p_{w}, q_{w}\right),\left(p^{\prime}, q^{\prime}\right)\right) ; r_{w}, R^{\prime}\right)=\left(T_{z}\left(p_{w}, q_{w}\right) ; r_{w}\right) \cup\left(T_{w}\left(q^{\prime}, p^{\prime}\right) ; R^{\prime}\right),
$$

with $\left(p^{\prime}, q^{\prime}\right)=\left(p_{z}-\delta \Delta p_{w}, q_{z}-\delta \Delta q_{w}\right)$ and $R^{\prime}=R_{z}-p_{z} q_{z}+p^{\prime} q^{\prime}=p^{\prime} q^{\prime}+r$.

Proof. The starting diagram is pulled back by $\Psi$ as

$$
(K ; r)=\left(l\left(q_{z}, p_{z}\right)[-1] ; r\right) \text { and }\left(K_{0} ; r_{0}\right)=\left(l\left(q_{w}, p_{w}\right)[0] ; \delta\right)
$$

in $T^{2} \times I$. We apply the handle-slides $(K ; r)$ over $\left(K_{0} ; r_{0}\right)$ in Lemma 5.1 . The result is the union of

$$
\left(K_{0} ; \delta\right)=\left(l\left(q_{w}, p_{w}\right)[0] ; \delta\right) \text { and }\left(K^{\prime} ; r^{\prime}\right)=\left(l\left(q_{z}-\delta \Delta q_{w}, p_{z}-\delta \Delta p_{w}\right)[+1] ; r\right) .
$$

Let $\iota$ be a self diffeomorphism of $I$ (isotopic to $\operatorname{id}_{I}$ ) satisfying $\iota(\tau)=\tau-1$ for $0 \leq \tau \leq 1$. Using the embedding $\Psi \circ\left(\mathrm{id}_{T^{2}} \times \iota\right): T^{2} \times I \rightarrow S^{3}$, we have the required framed link in $S^{3}$.

We apply Lemma 5.2 to reduce 4-dim diagrams constructed in Theorem 4.1, which depends on the sequence. By

$$
\left(T\left(\left(p_{z}, q_{z}\right),\left(p_{w}, q_{w}\right)\right) ; R_{z}, r_{w}\right) \rightarrow\left(T\left(\left(p_{w}, q_{w}\right),\left(p^{\prime}, q^{\prime}\right)\right) ; r_{w}, R^{\prime}\right),
$$

we denote that there exists a sequence of only handle-slides from the left hand-side diagram to the right hand-side one. In other words, they describe the same 4-manifold as 4-dim diagrams.

Lemma 5.3 (4-dim calculus) We can reduce the 4-dim diagrams as follows:

(1) $\left(T\left(\left(A_{i}, B_{i}\right),\left(A_{i-1}, B_{i-1}\right)\right) ; P_{i}, P_{i-1}\right) \rightarrow\left(T\left(\left(A_{i-1}, B_{i-1}\right),\left(A_{i-2}, B_{i-2}\right)\right) ; P_{i-1}, P_{i-2}\right)$.

(2) $\left(T\left(\left(S_{i}, T_{i}\right),\left(U_{i-1}, V_{i-1}\right)\right) ; P_{i}, P_{i-1}\right) \rightarrow\left(T\left(\left(U_{i-1}, V_{i-1}\right),\left(S_{i-2}, T_{i-2}\right)\right) ; P_{i-1}, P_{i-2}\right)$ and $\left(T\left(\left(U_{i}, V_{i}\right),\left(S_{i-1}, T_{i-1}\right)\right) ; P_{i}, P_{i-1}\right) \rightarrow\left(T\left(\left(S_{i-1}, T_{i-1}\right),\left(U_{i-2}, V_{i-2}\right)\right) ; P_{i-1}, P_{i-2}\right)$.

It also holds for the pairs in the sequence [stuv].

(3) $\left(T\left(\left(K_{i}, L_{i}\right),\left(K_{i-1}, L_{i-1}\right)\right) ; P_{i}, P_{i-1}\right) \rightarrow\left(T\left(\left(K_{i-1}, L_{i-1}\right),\left(K_{i-2}, L_{i-2}\right)\right) ; P_{i-1}, P_{i-2}\right)$.

It also holds for the pairs in the sequence $[\mathrm{klmn}]$.

Proof. We go into the detail for the proof in the case (1). Since $\Delta=A_{i} B_{i-1}-B_{i} A_{i-1}=a$ and the recursive formula $X_{i+1}=a X_{i}-X_{i-1}$ in Lemma 3.1(5) and (3), we can deform the diagram into $T\left(\left(A_{i-1}, B_{i}\right),\left(A_{i-2}, B_{i-2}\right)\right)$ by Lemma 5.2 , as unoriented link. In this case (and case $(3))$, orientation of the second component is changed, but it does not matter as a framed link. The framings are equal to the required ones.

In the other cases (2) and (3), the method of the proof is the same as that of (1). Only signs $\delta$, values $\Delta$ and recursive formulas are replaced with Table 5 . We have the lemma.

Proof (of Theorem 1.5, i.e, Theorem 1.12 and Theorem 1.18) Our main theorem follows from the result of 3-dim calculus in Theorem 4.1 by 4 -dim calculus in Lemma 5.3 inductively. In the case of the sequence $[A B C D]$, the diagram becomes a framed Hopf link $(H ; 0, a+2)$, where $H$ means a Hopf link. We use Lemma 4.4 of Kirby [K2]. In the case [STUV] or [stuv], after the reduction, the diagram becomes a framed Hopf link $(H ; 1,2)$. It is easy to see $X(H ; 1,2) \cong \mathbb{C} P^{2} \sharp \mathbb{C} P^{2}$. In the case $[K L M N]$ or $[k l m n]$, after the reduction, the diagram becomes a framed link $(T((1,6),(1,3)) ; 5,2)$ or $(T((1,6),(1,2)) ; 5,1)$. In either case, the 4-manifold is diffeomorphic to $\mathbb{C} P^{2} \sharp \mathbb{C} P^{2}$, see Figure 23. 


\begin{tabular}{|c|c|c|c|l|}
\hline Case & Sequence & $\delta$ & $\Delta$ & see \\
\hline$(1)$ & {$[A B C D]$} & -1 & $A_{i} B_{i-1}-B_{i} A_{i-1}=a$ & Lemma 3.1 \\
\hline$(2)$ & {$[S T U V]$} & -1 & $S_{i} V_{i-1}-T_{i} U_{i-1}=-1$ & \multirow{2}{*}{ Lemma 3.8 } \\
& & +1 & $U_{i} T_{i-1}-V_{i} S_{i-1}=1$ & \\
\hline$(2)$ & {$[s t u v]$} & -1 & $s_{i} v_{i-1}-t_{i} u_{i-1}=-2$ & Lemma 3.10 \\
& +1 & $u_{i} t_{i-1}-v_{i} s_{i-1}=2$ & \\
\hline$(3)$ & {$[K L M N]$} & -1 & $K_{i} L_{i-1}-L_{i} K_{i-1}=-3$ & Definition 1.16 \\
\hline$(3)$ & {$[k l m n]$} & -1 & $k_{i} l_{i-1}-l_{i} k_{i-1}=-4$ & Definition 1.16 \\
\hline
\end{tabular}

Table 5: Diagram Reduce

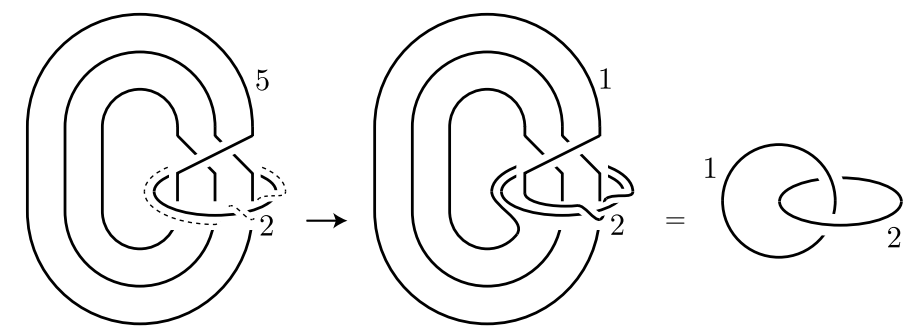

Figure 23: $X(T((1,6),(1,3)) ; 5,2) \cong \mathbb{C} P^{2} \sharp \mathbb{C} P^{2}$

\subsection{Demonstration in the case $i=2$}

Here, as a demonstration, we prove Theorem 4.1 and Theorem 1.12 in the case $i=2$ by concrete Kirby calculus. This was our starting example. Figures are just after the reference list.

Demonstration of Theorem 4.1(1) in the case $i=2$. We start with dualizing $k_{A B}^{*}$ in the diagram $\mathcal{L}(2)$, see the left top figure in Figure 25. We use an extended version of $(-2)$-formula (Subsection 2.2) in the form in Figure 24, where the gray band contains some components of the link, and the box with $(n+1)$ means $(n+1)$ full twists. The framing of each component in the gray band changes by $+(n+1)(l k)^{2}$, where $l k$ is the linking number of the component and the 0 -framed component. Next, we blow-down the $(+1)$-curve. Finally, we use the formula

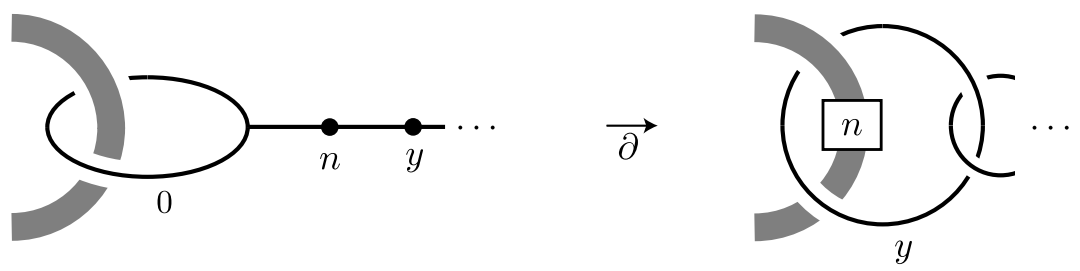

Figure 24: A formula

again. We have the required framed link $\left(T\left(\left(A_{2}, B_{2}\right),\left(A_{1}, B_{1}\right)\right) ; P_{2}, P_{1}\right)=\left(T\left(\left(a+1, a^{2}-a-\right.\right.\right.$ 1), $\left.(1, a-1)) ; a^{3}-2 a, a\right)$. 
Proof. (of Theorem 1.12 in the case $i=2$ ) We have to do Kirby calculus from the diagram in Figure 25 to that of the required manifold, only by handle-slides (K-I). See Kirby calculus in Figure 26. Handle-slides are indicated by thin dotted arrows. Finally, we use Lemma 4.4 of Kirby [K2].

\section{Completeness of the list}

Proof of Theorem 1.4 is divided into three cases. We look for pairs of lens space surgeries along torus knots

$$
M(T(X, Y) ; X Y \pm 1) \cong-L\left(X Y \pm 1, X^{2}\right),
$$

whose lens spaces are homeomorphic. We consider positive torus knots $X, Y>0$ (and $Z, W>$ 0 below). The cases are

(1) $M(T(X, Y) ; X Y+\epsilon) \cong M(T(Z, W) ; Z W+\delta)$, where $\epsilon, \delta= \pm 1$,

(2) $M(T(X, Y) ; X Y+1) \cong-M(T(Z, W) ; Z W-1)$,

(3) $M(T(X, Y) ; X Y+\epsilon) \cong-M(T(Z, W) ; Z W+\epsilon)$, where $\epsilon= \pm 1$.

They correspond to the classification $(1),(2),(3)$ in Section 1, and they are discussed in Subsections $6.1,6.2,6.3$, respectively.

In this section, a notation $((A, B),(C, D))$ (or $(A, B, C, D)$ for short) means a given pair of pairs (under consideration), does not mean a pair in the sequence $[A B C D]$.

Definition 6.1 According to symmetry of torus knots, we may change $((X, Y),(Z, W))$ to (itself or) one among

$$
((Y, X),(Z, W)),((X, Y),(W, Z)),((Y, X),(Z, W))
$$

mainly for uniqueness. When we do it, we say "we retake $((X, Y),(Z, W))$ ".

In the cases where we $X Y+\epsilon=Z W+\epsilon(=P)$, we say that a pair $((X, Y),(Z, W))$ is a trivial pair, if $(X, Y)=(Z, W)$ or $(X, Y)=(W, Z)$. We are concerned with non-trivial pairs.

Remark 6.2 Theorem 1.4 includes that there exist no pair in (1) with $\delta=\epsilon$ (i.e., $P=$ $X Y+\epsilon=Z W+\epsilon$ with $\epsilon= \pm 1$ ), and that there exist no non-trivial pair in (3) with $\epsilon=1$ (i.e., $P=X Y+1=Z W+1$ ).

Structures of the subsections are roughly sketched as follows:

1. Define a Condition $\mathcal{C}$ on a pair of pairs $((X, Y),(Z, W))$.

2. Define an operation Reduction $\mathcal{R}$, which gives a smaller pair from a given pair.

3. Make a list of the smallest pairs satisfying (some of) Condition C. They are Goals of reductions $\mathcal{R}$.

4. Study some invariants $(x, y, a, b, c)$ kept by reductions, and prove that reduced pairs also satisfy (some of) conditions. 
5. Prove that any given pair is reduced to a special pair in the list of Goals of reductions $\mathcal{R}$, by iteration of reductions.

6. Prove that the inverse operation from the goal back to the given pair agrees with the inductive definition of one five sequences.

The details of definitions and the orders (around 2,3,4) of the steps depend on the cases a little. For the cases (2) and (3), coprimeness will be considered at the end. The invariants decide the index $a$ of the sequence $[A B C D]$ in the case (1), decide the sequence $[S T U V]$ or $[s t u v]([K L M N]$ or $[k l m n]$, respectively) in the case (2) (or (3)).

For a real number $\alpha$, by $\lfloor\alpha\rfloor$ and $\lceil\alpha\rceil$, we denote the maximal integer that is not greater than $\alpha$, and the minimal integer that is not less than $\alpha$, respectively.

Definition 6.3 For positive integers, " $x<y$ implies $x^{2}<x y+1$ " always holds. On the other hand, " $x<y$ implies $x^{2}<x y-1$ " holds for almost all positive integers, but has a few exceptions: $(x, y)=(1,1),(1,2)$ and $(r, r)$ with $r>1$. We call these pairs exceptions.

\subsection{First case: Lens spaces with same orientations}

Suppose that positive torus knots $T(A, B)$ and $T(C, D)$ admit lens space surgeries whose lens spaces are orientation-preservingly homeomorphic: $M(T(A, B) ; A B+\epsilon) \cong M(T(C, D) ; C D+$ $\delta)$. Then the pair $((A, B),(C, D))$ satisfies the following condition $\mathcal{C} 1$. Here, we may retake the pair if necessary.

Definition 6.4 Let $\epsilon= \pm 1, \delta= \pm 1$. We say that a pair $((A, B),(C, D))$ satisfies Condition $\mathcal{C} 1$ with $(\epsilon, \delta)$ if it satisfies the followings:

(0) $(A, B)$ and $(C, D)$ are pairs of coprime positive integers.

(1) $A B+\epsilon=C D+\delta$. We call this number $P$.

(2) $0<A<B$ and $0<D<C$.

(3) $B^{2}-D^{2} \equiv 0 \bmod P$ and $C^{2}-A^{2} \equiv 0 \bmod P$.

We have some remarks: When $\delta=\epsilon$, the pair $((A, B),(C, D))$ can be a trivial pair. We should retake $((A, B),(C, D))$ at $(2)$, before (3). On (3), under (1), the second half of (3) follows from the first half. By (2), it holds that $A^{2}, D^{2}<P$ thus $A^{2} \not \equiv D^{2} \bmod P$ for non-trivial pairs.

Definition 6.5 (Reduction $\mathcal{R} 1$ ) For any pair $((A, B),(C, D))$ satisfying Condition $\mathrm{C} 1$ with $(\epsilon, \delta)$, we define a pair $\left(\left(A^{\prime}, B^{\prime}\right),\left(C^{\prime}, D^{\prime}\right)\right)$ as below: By using $x=\lfloor B / A\rfloor, y=\lfloor C / D\rfloor$,

$$
\left\{\begin{array} { l } 
{ A ^ { \prime } = C - y D } \\
{ B ^ { \prime } = D } \\
{ C ^ { \prime } = A } \\
{ D ^ { \prime } = B - x A }
\end{array} , \quad \text { whose inverse is } \left\{\begin{array}{l}
A=C^{\prime} \\
B=x C^{\prime}+D^{\prime} \\
C=y B^{\prime}+A^{\prime} \\
D=B^{\prime}
\end{array} .\right.\right.
$$

From the definition of $x$ and $y$, we have $0 \leq A^{\prime}<D=B^{\prime}$ and $0 \leq D^{\prime}<A=C^{\prime}$. We call this operation from $((A, B),(C, D))$ to $\left(\left(A^{\prime}, B^{\prime}\right),\left(C^{\prime}, D^{\prime}\right)\right)$ reduction. By reduction, the minimum of the four integers decreases strictly: $\min \{A, B, C, D\}>\min \left\{A^{\prime}, B^{\prime}, C^{\prime}, D^{\prime}\right\} \geq 0$. 
Lemma 6.6 (Goals of Reduction $\mathcal{R} 1)$ Suppose that $((A, B),(C, D))$ is reduced to $\left(\left(A^{\prime}, B^{\prime}\right),\left(C^{\prime}, D^{\prime}\right)\right)$. The following four conditions are mutually equivlent:

$$
\text { (i) } A^{\prime}=0, \quad \text { (ii) } D^{\prime}=0, \quad \text { (iii) } A=1, \quad \text { (iv) } D=1 \text {. }
$$

Proof. It is easy to see that (iii) implies (ii) and that (iv) implies (i). We show that (i) implies (iv) and (iii). Since (i) means that $C$ is divisible by $D$. By coprimeness and $D<C$ in Condition $\mathrm{C} 1(0)$ and (2), respectively, we have (iv) $D=1$. Hence $C^{2} \equiv D^{2}=1$ $\bmod P=C D \pm 1$. By $A^{2} \equiv C^{2} \equiv 1 \bmod P$ in Condition $\mathrm{C} 1(3)$ and $A^{2}<A B-1 \leq P$, we have $A^{2}=1$, as an integer. We have (iii) $A=1$. Similarly, we can show that (ii) implies (iii) and (iv).

Let $((A, B),(C, D))$ be a pair satisfying Condition $\mathcal{C} 1$ with $(\epsilon, \delta)$ and reduced to $\left(\left(A^{\prime}, B^{\prime}\right),\left(C^{\prime}, D^{\prime}\right)\right)$. For a while (until Lemma 6.10), we assume that $1<A$ and $1<D$. Hence we have $x<x A<B$ and $y<y D<C$. We have $0<A^{\prime}$ and $0<D^{\prime}$ also. Since $\operatorname{gcd}\left(A^{\prime}, B^{\prime}\right)=\operatorname{gcd}(C-y D, D)=$ $\operatorname{gcd}(C, D)=1$ and $\operatorname{gcd}\left(C^{\prime}, D^{\prime}\right)=\operatorname{gcd}(A, B-x A)=\operatorname{gcd}(A, B)=1,\left(A^{\prime}, B^{\prime}\right)$ and $\left(C^{\prime}, D^{\prime}\right)$ are pairs of coprime positive integers. It means that the reduced pair $\left(\left(A^{\prime}, B^{\prime}\right),\left(C^{\prime}, D^{\prime}\right)\right)$ satisfies (0) and (2) in Condition $\mathcal{C} 1$ without retaking.

\section{Lemma 6.7}

$$
\left\{\begin{array}{l}
B^{2}-D^{2}=x P \\
C^{2}-A^{2}=y P
\end{array}\right.
$$

where $x=\lfloor B / A\rfloor, y=\lfloor C / D\rfloor$. Thus we have $D<B$ and $A<C$.

Proof.

$$
\begin{aligned}
B^{2}-D^{2} & =B\left(x A+D^{\prime}\right)-D^{2}=x(A B+\epsilon)+B D^{\prime}-D^{2}-\epsilon x \\
& =x P+\left(B D^{\prime}-D^{2}-\epsilon x\right) .
\end{aligned}
$$

On $B^{2}-D^{2}-x P=B D^{\prime}-D^{2}-\epsilon x$, we can show the following inequalities:

$$
\begin{gathered}
\left(B D^{\prime}-D^{2}-\epsilon x\right) \leq B(A-1)-D^{2}-\epsilon x=A B-D^{2}-(B+\epsilon x)<P, \\
-\left(B D^{\prime}-D^{2}-\epsilon x\right)<D^{2}-x A D^{\prime}+\epsilon x=D^{2}-\left(A D^{\prime}+\epsilon\right) x<D^{2}<P .
\end{gathered}
$$

They mean

$$
-P<B D^{\prime}-D^{2}-\epsilon x<P .
$$

By the congruence in Condition $\mathcal{C} 1(3)$, we have $B^{2}-D^{2}-x P \equiv 0 \bmod P$. Thus we have $B^{2}-D^{2}-x P=0$.

Similarly, we can show $C^{2}-A^{2}=y P$ by

$$
\begin{aligned}
& C^{2}-A^{2}=C\left(y D+A^{\prime}\right)-A^{2}=y(C D-\delta)+C A^{\prime}-A^{2}+\delta y \\
&=y P+\left(C A^{\prime}-A^{2}+\delta y\right), \\
&\left(C A^{\prime}-A^{2}+\delta y\right) \leq C(D-1)-A^{2}+\delta y=C D-A^{2}-(C-\delta y)<P,
\end{aligned}
$$

and

$$
-\left(C A^{\prime}-A^{2}+\delta y\right)<A^{2}-y D A^{\prime}-\delta y=A^{2}-\left(D A^{\prime}+\delta\right) y<D^{2}<P .
$$




\section{Lemma 6.8}

(i) $y D^{2}-x A^{2}=2(\epsilon-\delta)$.

(ii) $A^{\prime} B^{\prime}-\delta=C^{\prime} D^{\prime}-\epsilon$. We call this number $P^{\prime}$.

(iii) $P^{\prime}=P-2 \delta-y D^{2}=P-2 \epsilon-x A^{2}$.

Proof.

$$
\begin{aligned}
A^{2} D^{2}-1 & =\left(C^{2}-y P\right) D^{2}-1=C^{2} D^{2}-y P D^{2}-1=(P-\delta)^{2}-1-y P D^{2} \\
& =P\left(P-2 \delta-y D^{2}\right), \\
A^{2} D^{2}-1 & =A^{2}\left(B^{2}-x P\right)-1=A^{2} B^{2}-x P A^{2}-1=(P-\epsilon)^{2}-1-x P A^{2} \\
& =P\left(P-2 \epsilon-x A^{2}\right) .
\end{aligned}
$$

Thus (i) $-2 \delta-y D^{2}=-2 \epsilon-x A^{2}$ holds.

$$
\begin{aligned}
& A^{\prime} B^{\prime}-\delta=(C-y D) D-\delta=C D-y D^{2}-\delta=P-y D^{2}-2 \delta=P^{\prime}, \\
& C^{\prime} D^{\prime}-\epsilon=A(B-x A)-\epsilon=A B-x A^{2}-\epsilon=P-x A^{2}-2 \epsilon=P^{\prime} .
\end{aligned}
$$

Thus we have (ii) and (iii).

\section{Lemma 6.9}

$$
\left\{\begin{array}{l}
\left(B^{\prime}\right)^{2}-\left(D^{\prime}\right)^{2}=x P^{\prime} \\
\left(C^{\prime}\right)^{2}-\left(A^{\prime}\right)^{2}=y P^{\prime}
\end{array},\right.
$$

where $x=\lfloor B / A\rfloor, y=\lfloor C / D\rfloor$. Thus we have $D^{\prime}<B^{\prime}$ and $A^{\prime}<C^{\prime}$.

Proof.

$$
\begin{aligned}
\left(B^{\prime}\right)^{2}-\left(D^{\prime}\right)^{2} & =D^{2}-(B-x A)^{2}=D^{2}-B^{2}+2 x A B-x^{2} A^{2} \\
& =-x P+2 x(P-\epsilon)-x^{2} A^{2} \\
& =x\left(P-2 \epsilon-x A^{2}\right)=x P^{\prime} \\
\left(C^{\prime}\right)^{2}-\left(A^{\prime}\right)^{2} & =A^{2}-(C-y D)^{2}=A^{2}-C^{2}+2 y C D-y^{2} D^{2} \\
& =-y P+2 y(P-\delta)-y^{2} D^{2} \\
& =y\left(P-2 \delta-y D^{2}\right)=y P^{\prime}
\end{aligned}
$$

Lemma 6.10 Let $((A, B),(C, D))$ be a pair satisfying Condition $\mathrm{C} 1$ with $(\epsilon, \delta)$ and reduced to $\left(\left(A^{\prime}, B^{\prime}\right),\left(C^{\prime}, D^{\prime}\right)\right)$. Then either $(A)$ or $(B)$ holds:

(A) It is a goal of reductions with $A^{\prime}=D^{\prime}=0$.

(B) $\left(\left(A^{\prime}, B^{\prime}\right),\left(C^{\prime}, D^{\prime}\right)\right)$ satisfies Condition $\mathcal{C} 1$ with $(-\delta,-\epsilon)$ without retaking. Furthermore, $x^{\prime}=\left\lfloor B^{\prime} / A^{\prime}\right\rfloor, y^{\prime}=\left\lfloor C^{\prime} / D^{\prime}\right\rfloor$ for the next reduction is equal to $x=\lfloor B / A\rfloor, y=\lfloor C / D\rfloor$ in the reduction, respectively. 
Proof. If $A^{\prime}=0$ or $D^{\prime}=0$, then it is in the case (A) by Lemma 6.6. Otherwise, we have already shown that the reduced pair $\left(\left(A^{\prime}, B^{\prime}\right),\left(C^{\prime}, D^{\prime}\right)\right)$ satisfies Condition $\mathrm{C} 1(0)$ and $(2)$ with $(-\delta,-\epsilon)$, just before Lemma 6.7. The remaining conditions (1), (3) follow from Lemma 6.8 (ii), Lemma 6.9, respectively. By Lemma 6.7 (applied to $\left.\left(\left(A^{\prime}, B^{\prime}\right),\left(C^{\prime}, D^{\prime}\right)\right)\right)$ and Lemma 6.9, we have $x^{\prime}=\left\{\left(B^{\prime}\right)^{2}-\left(D^{\prime}\right)^{2}\right\} / P^{\prime}=x$ and $y^{\prime}=\left\{\left(C^{\prime}\right)^{2}-\left(A^{\prime}\right)^{2}\right\} / P^{\prime}=y$.

Theorem 6.11 Suppose that a pair $((A, B),(C, D))$ satisfies Condition $\mathfrak{C}_{1}$ with $(\epsilon, \delta)$. If $\epsilon=\delta$, then the pair is a trivial pair. For the case $\epsilon \neq \delta$, we may assume $(\epsilon, \delta)=(1,-1)$, by considering $((D, C),(A, B))$ instead of $((A, B),(C, D))$ if necessary. Then, $((A, B),(C, D))$ is in the sequence $[A B C D]$ (see Definition 1.10), i.e., there exist positive integers a and $i$ such that

$$
A=A(a, i), B=B(a, i), C=C(a, i), D=D(a, i)
$$

Proof. We iterate reductions on the given pair $((A, B),(C, D))=\left(\left(A_{(0)}, B_{(0)}\right),\left(C_{(0)}, D_{(0)}\right)\right)$. By $\left(\left(A_{(k)}, B_{(k)}\right),\left(C_{(k)}, D_{(k)}\right)\right)$, we denote the result of the $k$-th reduction. Note that we do not retake the pairs, and that $x$ and $y$ are constant in the iteration, by Lemma 6.10 (B). Since $\min \left\{A_{(k)}, B_{(k)}, C_{(k)}, D_{(k)}\right\}$ are non-negative and decrease strictly with respect to $k$, after finite number of reductions, say at the $(n+1)$-th reduction, Case (A) occurs: $A_{(n+1)}=D_{(n+1)}=0$. By Lemma 6.6, we have $A_{(n)}=D_{(n)}=1$.

Case $\epsilon=\delta$. By Lemma 6.8 (i), we have $y=x$. Thus, by the inverse of Reduction $\mathcal{R} 1$, all pairs in the process are trivial pairs.

Case $(\epsilon, \delta)=(1,-1)$. Note that $(-\delta,-\epsilon)=(\epsilon, \delta)=(1,-1)$. By Lemma 6.8 (i), we have $y-x=4$. We define $a=y-2=x+2$. It holds that $B_{(n)}+1=C_{(n)}-1=P_{(n)}$ (from $\left.A_{(n)} B_{(n)}+1=C_{(n)} D_{(n)}-1=P_{(n)}\right)$. By Lemma $6.9,\left(B_{(n)}\right)^{2}-1=x P_{(n)}$. Thus $B_{(n)}-1=$ $x=a-2$ and $C_{(n)}=a+1$. We have $\left(\left(A_{(n)}, B_{(n)}\right),\left(C_{(n)}, D_{(n)}\right)\right)=((1, a-1),(a+1,1))$, which is in the sequence $[A B C D]$ with $i=1$. The inverse of Reduction $\mathcal{R} 1$ with $x=a-2, y=a+2$ agrees with the construction of the sequence $[A B C D]:\left(\left(A_{(k)}, B_{(k)}\right),\left(C_{(k)}, D_{(k)}\right)\right)$ is in the sequence $[A B C D]$ with $i=n-k+1(k=0,1, \ldots, n)$. We have the lemma.

\subsection{Second case: Lens spaces with opposite orientations I}

Suppose that positive torus knots $T(S, T)$ and $T(U, V)$ admit lens space surgeries whose lens spaces are orientation-preservingly homeomorphic as $M(T(S, T) ; S T+1) \cong-M(T(U, V) ; U V-$ $1)$. Then the pair $((S, T),(U, V))$ satisfies the following condition $\mathrm{C} 2$. Here, we may retake the pair if necessary.

Definition 6.12 We say that a pair $((S, T),(U, V))$ satisfies Condition $\mathcal{C} 2$ if it satisfies the followings:

(0) $(S, T)$ and $(U, V)$ are coprime pairs of positive integers.

(1) $S T+1=U V-1$. We call this number $P$.

(2) $S^{2}+V^{2} \equiv 0 \bmod P$ and $T^{2}+U^{2} \equiv 0 \bmod P$.

(3) $\min \{S, T, U, V\}=S$ or $\min \{S, T, U, V\}=U$. Possibly both (and $S=U$ ) hold. 
We have some remarks: On (2), under (1), the second half of (2) follows from the first half. We can retake a given pair $((S, T),(U, V))$ to one satisfying $(2)$ and (3) uniquely, see Definition 6.1. For example, $((S, T),(U, V))=((3,4),(7,2))$ is retaken to $((4,3),(2,7))$. We postpone considering coprimeness in (0) untill Lemma 6.22, as "Condition $\mathrm{C} 2$ (1)-(3)".

By Condition $\mathrm{C} 2(2)$, we can define positive integers $a, b$ by

$$
S^{2}+V^{2}=a P, \quad T^{2}+U^{2}=b P .
$$

We often use a logic " $S<T$ implies $S^{2}<P$ and $V<U$ implies $V^{2}<P$ thus $S^{2}+V^{2} \equiv 0$ $\bmod P$ implies $S^{2}+V^{2}=P$ (i.e., $a=1$ ) as integers (since $S^{2}+V^{2}<2 P$ )". But, there exist exceptions: $(U, V)=(1,1),(1,2)$ or $(r, r)$ with $r>1$, see Definition 6.3. Here, we list some small cases.

Lemma 6.13 (Goals of Reduction $\mathcal{R} 2)$ Pairs $((S, T),(U, V))$ satisfying Condition $\mathcal{C} 2$ (1)-(3) with $\min \{S, U\} \leq 2$ are listed as follows:

$$
\begin{aligned}
(S, T, U, V)= & (1,1,1,3),(1,1,3,1),(1,4,2,3),(1,4,3,2),(4,3,2,7), \\
& (2,2,6,1),(2,14,6,5), \\
& \left(r, r, 1, r^{2}+2\right),\left(r, 4 r, 2,2 r^{2}+1\right), \quad(r>1) .
\end{aligned}
$$

Here, only pairs in the first line satisfy coprimeness condition (0) in Condition $\mathcal{C} 2$.

Proof. It is easy to see that there is no integer solution in the cases of exceptions $(U, V)=$ $(1,1),(1,2),(2,1)$ and $U=V(>1)$. Thus we assume $U \neq V$. The proof is divided into four cases, eight subcases.

Case $S=1$ : Then $P=S T+1=T+1=U V-1$.

[Subcase $V<U]$ We have $S^{2}+V^{2}=1+V^{2}=P$. Here we used that $S^{2}+V^{2} \equiv 0 \bmod$ $P$ and $S^{2}+V^{2}<2 P$. By $U V-1=V^{2}+1$, we have $U=V+2 / V$, thus $V=1$ or $V=2$. Hence $(S, T, U, V)=(1,1,3,1),(1,4,3,2)$, respectively.

[Subcase $U<V$ ] We have $U^{2}<U V-1=P=T+1$. By $(T-1) P<T^{2}<T P$, we have $(T-1) P<T^{2}+U^{2}<(T+1) P$. Thus $T^{2}+U^{2} \equiv 0 \bmod P$ implies $T^{2}+U^{2}=T P$, thus $U^{2}=T$. By $U V-1=T+1=U^{2}+1$, we have $V=U+2 / U$, thus $U=1$ or $U=2$. Hence $(S, T, U, V)=(1,1,1,3),(1,4,2,3)$, respectively.

Case $S=2$ : Then $P=2 T+1=U V-1$.

[Subcase $V<U]$ We have $4+V^{2}=P$. By $U V-1=4+V^{2}$, we have $U=V+5 / V$, thus $V=1$ or $V=5$. Hence $(S, T, U, V)=(2,2,6,1),(2,14,6,5)$, respectively.

[Subcase $U<V]$ We have $U^{2}<U V-1=P=2 T+1$. By $(2 T-1) P<4 T^{2}<$ $2 T P$, we have $(2 T-1) P<4\left(T^{2}+U^{2}\right)<(2 T+4) P$. Thus $T^{2}+U^{2} \equiv 0 \bmod P$ implies either $2\left(T^{2}+U^{2}\right)=P T$ or $2\left(T^{2}+U^{2}\right)=(T+1) P$. In the first case, $2 U^{2}=T$, thus $U V-1=2 T+1=4 U^{2}+1$. We have $V=4 U+2 / U$, thus $U=1$ or $U=2$. Hence $(S, T, U, V)=(2,2,1,6),(2,8,2,9)$. They are $\left(r, r, 1, r^{2}+2\right),\left(r, 4 r, 2,2 r^{2}+1\right)$ with $r=2$. In the second case, $2 U^{2}=3 T+1$, thus $3(U V-1)=3(2 T+1)=4 U^{2}+1$. This has no integer solution.

Case $U=1$ : Then $P=U V-1=V-1=S T+1$.

[Subcase $T \leq S$ ] We have $T^{2}+1=P$. By $S T+1=T^{2}+1$, we have $S=T=1$. We go back to the case $S=1$. 
[Subcase $S \leq T$ ] We have $S^{2}<S T+1=P=V-1$. By $(V+1) P<V^{2}<(V+2) P$, we have $(V+1) P<S^{2}+V^{2}<(V+3) P$. Thus $S^{2}+V^{2} \equiv 0 \bmod P$ implies $S^{2}+V^{2}=(V+2) P$, thus $S^{2}=V-2, S T+1=V-1=S^{2}+1$. We have $T=S$ and $(S, T, U, V)=\left(r, r, 1, r^{2}+2\right)$ for any positive integer $r$.

Case $U=2$ : Then $P=2 V-1=S T+1$.

[Subcase $T \leq S$ ] We have $T^{2}+4=P$. By $S T+1=T^{2}+4, S=T+3 / T$, thus $T=1$ or $T=3$. Thus $(S, T, U, V)=(4,1,2,3),(4,3,2,7)$, respectively. $(4,1,2,3)$ does not satisfy $(3)$.

[Subcase $S \leq T$ ] We have $S^{2}<S T+1=P=2 V-1$. By $(2 V+1) P<4 V^{2}<2(V+1) P$, we have $(2 V+1) P<4\left(S^{2}+V^{2}\right)<(2 V+6) P$. Thus $S^{2}+V^{2} \equiv 0 \bmod P$ implies either $2\left(S^{2}+V^{2}\right)=(V+1) P$ or $2\left(S^{2}+V^{2}\right)=(V+2) P$. In the first case, we have $2 S^{2}=V-1$, thus $S T+1=2 V-1=4 S^{2}+1$. Hence $(S, T, U, V)=\left(r, 4 r, 2,2 r^{2}+1\right)$ for any positive integer $r$. In the second case, we have $2 S^{2}=3 V-2$, thus $3(S T+1)=3(2 V-1)=4 S^{2}+1$. This has no integer solution.

Now, we assume that $\min \{S, T, U, V\}>2$. Then Condition $\mathrm{C} 2$ (1) can be regarded as " $S T$ is nearly equal to $U V$ ". By Condition $\mathrm{C} 2$ (3), the order of $S, T, U, V$ is one of the followings (see Proposition 3.9 and Proposition 3.11). In accordance with the order of them, we classify pairs into four types:

$$
\begin{array}{ll}
\text { (Type S) } \quad S \leq V<U \leq T, & \text { (Type U) } U \leq T<S \leq V, \\
\text { (Type s) } S \leq U<V \leq T, & \text { (Type u) } U \leq S<T \leq V,
\end{array}
$$

If integers $k, l, m$ and $n$ satisfy $\min \{k, l, m, n\}>2$ and $|m n-k l| \leq 2$, then $k=m$ implies $l=n$. Thus, if $((S, T),(U, V))$ is a non-trivial pair, we can replace every " $\leq$ " by " $<$ " above.

Lemma 6.14 Let $\epsilon= \pm 1, \delta= \pm 1$. Let $(k, l),(m, n)$ be pairs of coprime positive integers with $l, n>2$. If $k l+\epsilon=m n+\delta$, then $\lfloor m / l\rfloor=\lfloor k / n\rfloor$.

Proof. In the case $\epsilon=\delta$, it is trivial. By the symmetry, we only have to consider the case $\epsilon=1, \delta=-1$, i.e., $m n-k l=2$. Then

$$
\frac{m}{l}-\frac{k}{n}=\frac{m n-k l}{l n}=\frac{2}{l n}<\frac{1}{n}, \text { thus } \frac{k+1}{n}>\frac{m}{l}>\frac{k}{n} .
$$

There exists no integer $N$ satisfying $(k+1) / n<N<k / n$. If either $(k+1) / n$ or $k / n$ is an integer, then $\lfloor m / l\rfloor=\lfloor k / n\rfloor$. We have the lemma.

Corollary 6.15 Let $((S, T),(U, V))$ be any pair satisfying Condition $\mathcal{C} 2(1)-(3)$ and $\min \{S, T, U, V\}>$ 2. Then we have $\lfloor V / S\rfloor=\lfloor T / U\rfloor$.

Definition 6.16 (Reduction $\mathcal{R} 2)$ For any pair $((S, T),(U, V))$ satisfying Condition $\mathrm{C} 2(1)$ (3) and $\min \{S, T, U, V\}>2$, we define a pair $\left(\left(S^{\prime}, T^{\prime}\right),\left(U^{\prime}, V^{\prime}\right)\right)$ as below: By using $x=$ $\lfloor V / S\rfloor=\lfloor T / U\rfloor$ (see Corollary 6.15),

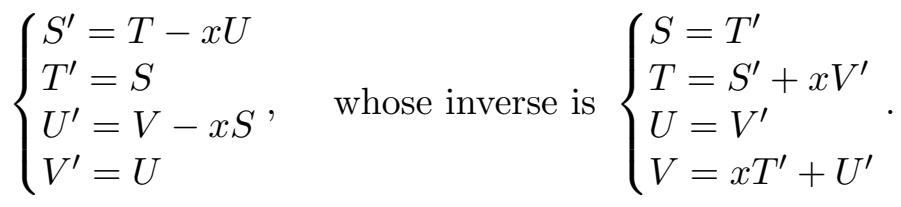

We call this operation from $((S, T),(U, V))$ to $\left(\left(S^{\prime}, T^{\prime}\right),\left(U^{\prime}, V^{\prime}\right)\right)$ reduction. By reduction, the minimum of four integers decreases strictly: $\min \{S, U\}>\min \left\{S^{\prime}, U^{\prime}\right\} \geq 0$. 
Lemma 6.17 For any pair $((S, T),(U, V))$ satisfying Condition $\mathrm{C} 2(1)$-(3) and $\min \{S, T, U, V\}>$ 2 , its reduction $\left(\left(S^{\prime}, T^{\prime}\right),\left(U^{\prime}, V^{\prime}\right)\right)$ satisfies Condition $\mathcal{C} 2(1)$ and (3) without retaking.

Proof. In Definition 6.16, we defined $x=\lfloor T / U\rfloor=\lfloor V / S\rfloor$. Thus $0 \leq S^{\prime}<U=V^{\prime}, 0 \leq U^{\prime}<$ $S=T^{\prime}$, and we have Condition $\mathrm{C} 2(3)$ :

$$
\min \left\{S^{\prime}, T^{\prime}, U^{\prime}, V^{\prime}\right\}=S^{\prime} \text { or } \min \left\{S^{\prime}, T^{\prime}, U^{\prime}, V^{\prime}\right\}=U^{\prime}
$$

Condition $\mathrm{C} 2(1)$ on $\left(\left(S^{\prime}, T^{\prime}\right),\left(U^{\prime}, V^{\prime}\right)\right)$ is verified by

$$
S^{\prime} T^{\prime}=(T-x U) S=S T-x S U, \quad U^{\prime} V^{\prime}=(V-x S) U=U V-x S U
$$

and $S T+1=U V-1=P$. We set $P^{\prime}=P-x S U$.

Next, we use an identity

$$
\left(S^{2}+V^{2}\right)\left(T^{2}+U^{2}\right)=(T V-S U)^{2}+(S T+U V)^{2}
$$

By Condition $\mathrm{C} 2(1)$, we have $S T+U V=2 P$. By Condition $\mathrm{C} 2(2)$ and $(6.1),(T V-S U) / P$ is also a positive integer. We let $c$ denote this integer:

$$
T V-S U=c P
$$

Using this, we have

$$
\left(\begin{array}{cc}
S & V \\
V & -S
\end{array}\right)\left(\begin{array}{l}
T \\
U
\end{array}\right)=\left(\begin{array}{l}
2 P \\
c P
\end{array}\right), \quad\left(\begin{array}{cc}
U & T \\
T & -U
\end{array}\right)\left(\begin{array}{l}
V \\
S
\end{array}\right)=\left(\begin{array}{l}
2 P \\
c P
\end{array}\right)
$$

\section{Lemma 6.18}

(1) $c=x$, where $x=\lfloor V / S\rfloor=\lfloor T / U\rfloor$.

(2) $a b=x^{2}+4$.

(3)

$$
\begin{array}{ll}
a T=2 S+x V, & b V=2 U+x T, \\
a U=-x S+2 V, & b S=-x U+2 T .
\end{array}
$$

Proof. (1) The following equalities holds:

$$
\begin{gathered}
\frac{T V}{P}=\frac{2 T V}{S T+U V}<\frac{1}{2}\left(\frac{V}{S}+\frac{T}{U}\right)<x+1, \\
2 T V-2 x P=2 T V-x(S T+U V)=T(V-x S)+V(T-x U)>0 .
\end{gathered}
$$

Thus we have $x P<T V<(x+1) P$. Since $0<S U<P$, we have (1).

(2) By the identitiy (6.2) and the definitions (6.1), (6.3) of $a, b, c$, we have $a b=c^{2}+4=$ $x^{2}+4$. The linear relations (3) follow from (6.1), (6.4) and (1), by multiplying the inverse matrices. 
Lemma 6.19 For any pair $((S, T),(U, V))$ satisfying Condition $\mathcal{C} 2(1)-(3)$ and $\min \{S, T, U, V\}>$ 2 , its reduction $\left(\left(S^{\prime}, T^{\prime}\right),\left(U^{\prime}, V^{\prime}\right)\right)$ satisfies Condition $\mathcal{C} 2$ (2). More precisely,

$$
\left(S^{\prime}\right)^{2}+\left(V^{\prime}\right)^{2}=b P^{\prime}, \quad\left(T^{\prime}\right)^{2}+\left(U^{\prime}\right)^{2}=a P^{\prime} .
$$

Proof. By (6.4),

$$
\begin{aligned}
\left(S^{\prime}\right)^{2}+\left(V^{\prime}\right)^{2} & =(T-x U)^{2}+U^{2}=T^{2}+U^{2}-x U(2 T-x U) \\
& =T^{2}+U^{2}-x U(2 T-c U)=b(P-x S U) \\
& =b P^{\prime} . \\
\left(T^{\prime}\right)^{2}+\left(U^{\prime}\right)^{2} & =S^{2}+(V-x S)^{2}=S^{2}+V^{2}-x S(2 V-x S) \\
& =S^{2}+V^{2}-x S(2 V-c S)=a(P-x S U) \\
& =a P^{\prime} .
\end{aligned}
$$

We can summarize the argument above:

Lemma 6.20 Let $((S, T),(U, V))$ be a pair satisfying Condition $\mathcal{C} 2(1)-(3)$ and $\min \{S, T, U, V\}>$ 2. We let $\left(\left(S^{\prime}, T^{\prime}\right),\left(U^{\prime}, V^{\prime}\right)\right)$ denote its reduction. Then either $(A)$ or $(B)$ holds:

(A) It is one of Goals of Reduction $\mathcal{R} 2$, i.e., $\min \left\{S^{\prime}, T^{\prime}, U^{\prime}, V^{\prime}\right\} \leq 2$.

(B) $\left(\left(S^{\prime}, T^{\prime}\right),\left(U^{\prime}, V^{\prime}\right)\right)$ satisfies Condition $\mathcal{C} 2(1)$-(3) without retaking. Furthermore, integers $a^{\prime}, b^{\prime}, x^{\prime}$ defined by

$$
x^{\prime}=\left\lfloor V^{\prime} / S^{\prime}\right\rfloor=\left\lfloor T^{\prime} / U^{\prime}\right\rfloor, \quad\left(S^{\prime}\right)^{2}+\left(V^{\prime}\right)^{2}=a^{\prime} P^{\prime}, \quad\left(T^{\prime}\right)^{2}+\left(U^{\prime}\right)^{2}=b^{\prime} P^{\prime}
$$

for the next reduction of $\left(\left(S^{\prime}, T^{\prime}\right),\left(U^{\prime}, V^{\prime}\right)\right)$ and integers $a, b, x$ defined by

$$
x=\lfloor V / S\rfloor=\lfloor T / U\rfloor, \quad S^{2}+V^{2}=a P, \quad T^{2}+U^{2}=b P
$$

of $((S, T),(U, V))$ satisfy

$$
x^{\prime}=x, \quad a^{\prime}=b, \quad b^{\prime}=a .
$$

Proof. Suppose that it is not the case (A), i.e., $\min \left\{S^{\prime}, T^{\prime}, U^{\prime}, V^{\prime}\right\}>2$. Almost all have been proved in Lemma 6.17 and Lemma 6.19. The integers $a^{\prime}, b^{\prime}$ are defined by Definition 6.16 and (6.1) (applying to $\left.\left(\left(S^{\prime}, T^{\prime}\right),\left(U^{\prime}, V^{\prime}\right)\right)\right)$. They are equal to $b, a$ of $((S, T),(U, V))$ by Lemma 6.19 , respectively. The equality $x^{\prime}=x$ follows from $\left(x^{\prime}\right)^{2}+4=a^{\prime} b^{\prime}=b a=x^{2}+4$ by Lemma 6.18 .

We focus on coprimeness Condition $\mathrm{C} 2(0)$.

Lemma 6.21 Suppose that $((S, T),(U, V))$ is a pair satisfying Condition $\mathrm{C}_{2}$ (1)-(3) and $\min \{S, T, U, V\}>2$, and $\left(\left(S^{\prime}, T^{\prime}\right),\left(U^{\prime}, V^{\prime}\right)\right)$ is a reduction of $((S, T),(U, V))$. If $\operatorname{gcd}\left(S^{\prime}, T^{\prime}, x\right)>$ 1 , then $\operatorname{gcd}(S, T, x)>1$, where $x=\lfloor V / S\rfloor=\lfloor T / U\rfloor$.

Proof. It is easy to see by the inverse of Reduction $\mathcal{R} 2$ in Definition 6.16 . 
Lemma 6.22 Let $((S, T),(U, V))$ be a pair satisfying Condition $\mathcal{C} 2$ (including (0)) and $\min \{S, T, U, V\}>2$. If its reduction $\left(\left(S^{\prime}, T^{\prime}\right),\left(U^{\prime}, V^{\prime}\right)\right)$ satisfies $\min \left\{S^{\prime}, T^{\prime}, U^{\prime}, V^{\prime}\right\} \leq 2$, then either (i) or (ii) holds:

(i) $\left(\left(S^{\prime}, T^{\prime}\right),\left(U^{\prime}, V^{\prime}\right)\right)=((4,3),(2,7))$ and $x=1$.

(ii) $\left(\left(S^{\prime}, T^{\prime}\right),\left(U^{\prime}, V^{\prime}\right)\right)=((1,4),(2,3))$ and $x=2$.

Proof. Note that $\left(\left(S^{\prime}, T^{\prime}\right),\left(U^{\prime}, V^{\prime}\right)\right)$ satisfies Condition $\mathcal{C} 2(1)-(3)$ by Lemma 6.17 and the equalities in Lemma 6.19. Thus $x^{\prime}=x$ also holds. We go back to the list in Lemma 6.22.

Claim 1. $\min \left\{T^{\prime}, V^{\prime}\right\}>2$.

Since $S=T^{\prime}, U=V^{\prime}$ in the reduction, $\min \left\{T^{\prime}, V^{\prime}\right\} \leq 2$ contradicts to the assumption $\min \{S, T, U, V\}>2$.

Hence neither $\left(S^{\prime}, T^{\prime}, U^{\prime}, V^{\prime}\right)=(1,4,3,2)$ nor $(2,2,1,6)\left(\right.$ as $\left(r, r, 1, r^{2}+2\right)$ with $\left.r=2\right)$ occur.

Claim 2. $S^{\prime} T^{\prime} \neq 0$. (It means that neither $T / U$ nor $V / S$ is an integer.)

If $S^{\prime} T^{\prime}=0$, then $U^{\prime} V^{\prime}=2$ thus $U=V^{\prime} \leq 2$, which contradicts to the previous claim.

By the claims, only five cases are left in the list in Lemma 6.22. We study the integers $a^{\prime}, b^{\prime}$ and $x^{\prime}$ one by one.

Case 1. $\left(S^{\prime}, T^{\prime}, U^{\prime}, V^{\prime}\right)=(1,4,2,3)$ with $a^{\prime}=2, b^{\prime}=4, x^{\prime}=2$.

Case 2. $\left(S^{\prime}, T^{\prime}, U^{\prime}, V^{\prime}\right)=(4,3,2,7)$ with $a^{\prime}=5, b^{\prime}=1, x^{\prime}=1$.

Case 3. $\left(S^{\prime}, T^{\prime}, U^{\prime}, V^{\prime}\right)=(2,14,6,5)$ with $a^{\prime}=1, b^{\prime}=8, x^{\prime}=2$.

Case 4. $\left(S^{\prime}, T^{\prime}, U^{\prime}, V^{\prime}\right)=\left(r, r, 1, r^{2}+2\right)$ with $a^{\prime}=r^{2}+4, b^{\prime}=1$, thus $x^{\prime}=r$.

Case 5. $\left(S^{\prime}, T^{\prime}, U^{\prime}, V^{\prime}\right)=\left(r, 4 r, 2,2 r^{2}+1\right)$ with $a^{\prime}=r^{2}+1, b^{\prime}=4$, thus $x^{\prime}=2 r$.

The last three cases contradict coprimeness Condition $\mathrm{C} 2(0)$ on $((S, T),(U, V))$ by Lemma 6.21.

Theorem 6.23 Any pair $((S, T),(U, V))$ that satisfies Condition $\mathcal{C} 2$ is in the sequence $[S T U V]$ or $\left[\right.$ stuv] (see Definition 1.13). In other words, $((S, T),(U, V))=\left(\left(S_{i}, T_{i}\right),\left(U_{i}, V_{i}\right)\right)$ in the sequence $[S T U V]$, or $((S, T),(U, V))=\left(\left(s_{i}, t_{i}\right),\left(u_{i}, v_{i}\right)\right)$ in the sequence [stuv], for a positive integer $i$.

Proof. We iterate reductions on the given pair $((S, T),(U, V))=\left(\left(S_{(0)}, T_{(0)}\right),\left(U_{(0)}, V_{(0)}\right)\right)$. By $\left(\left(S_{(k)}, T_{(k)}\right),\left(U_{(k)}, V_{(k)}\right)\right)$, we denote the result of the $k$-th reduction. Note that we do not retake the pairs, and that $x$ is constant in the iteration, by Lemma 6.20 (B). Since $\min \left\{S_{(k)}, T_{(k)}, U_{(k)}, V_{(k)}\right\}$ are non-negative and decrease strictly with respect to $k$, after finite number of reductions, say at the $n$-th reduction, Case (A) occurs.

The goal $\left(\left(S_{(n)}, T_{(n)}\right),\left(U_{(n)}, V_{(n)}\right)\right)$ is $((4,3),(2,7))$ (with $\left.x=1\right)$ or $((1,4),(2,3))$ (with $x=2)$, otherwise all pairs in the reductions are not coprime, by an extension of Lemma 6.22. Note that $((4,3),(2,7))=\left(\left(S_{3}, T_{3}\right),\left(U_{3}, V_{3}\right)\right)$ in $[S T U V]$ and $((1,4),(2,3))=\left(\left(s_{1}, t_{1}\right),\left(u_{1}, v_{1}\right)\right)$ in $[$ stuv]. The inverse of Reduction $\mathcal{R} 2$ with $x=1$ or 2 agrees with the construction of the sequence $[S T U V]$ or $[s t u v]$, respectively.

In the first case, the pair $\left(\left(S_{(k)}, T_{(k)}\right),\left(U_{(k)}, V_{(k)}\right)\right)=\left(\left(S_{i}, T_{i}\right),\left(U_{i}, V_{i}\right)\right)$ with $i=n-k+3$ in $[S T U V]$. In the second case, $\left(\left(S_{(k)}, T_{(k)}\right),\left(U_{(k)}, V_{(k)}\right)\right)=\left(\left(s_{i}, t_{i}\right),\left(u_{i}, v_{i}\right)\right)$ with $i=n-k+1$ in $[$ stuv $]$. We have the lemma. 


\subsection{Third case: Lens spaces with opposite orientations II}

Suppose that positive torus knots $T(K, L)$ and $T(M, N)$ admit lens space surgeries whose lens spaces are orientation-reversingly homeomorphic as $M(T(K, L) ; K L+\epsilon) \cong-M(T(M, N) ; M N+$ $\epsilon)$. Then the pair $((K, L),(M, N))$ satisfies the following condition $\mathcal{C} 3$. Here, we may retake the pair if necessary.

Definition 6.24 Let $\epsilon= \pm 1$. We say that a pair $((K, L),(M, N))$ satisfies Condition $\mathcal{C} 3$ with $\epsilon$, if it satisfies the followings:

(0) $(K, L)$ and $(M, N)$ are pairs of coprime positive integers.

(1) $K L+\epsilon=M N+\epsilon$. We call this number $P$.

(2) $K^{2}+M^{2} \equiv 0 \bmod P$ and $L^{2}+N^{2} \equiv 0 \bmod P$.

(3) $\min \{K, L\} \leq \min \{M, N\}$ and $K \leq L$. (Thus $K=\min \{K, L, M, N\})$

We have some remarks: On (2), under (1), the second half follows from the first half. If a given pair $((S, T),(U, V))$ is non-trivial, we can retake it to one satisfying (2) and (3) uniquely, see Definition 6.1. We postpone considering coprimeness in (0) untill Lemma 6.32.

By Condition $\mathcal{C} 3(2)$, we can define positive integers $a, b$ by

$$
K^{2}+M^{2}=a P, \quad L^{2}+N^{2}=b P .
$$

Here, we list the smallest cases.

Lemma 6.25 (Goals of Reduction $\mathcal{R} 3$ ) Pairs $((K, L),(M, N))$ satisfying Condition $\mathcal{C} 3$ (1)(3) with $K \leq 2$ are listed as follows:

(1) Case $\epsilon=+1$ :

$$
\begin{aligned}
(K, L, M, N)= & (1,1,1,1),(2,6,3,4), \\
& \left(1, m^{2}, m, m\right),\left(2,2 n^{2}, 4 n, n\right), \quad(m>1, n>1) .
\end{aligned}
$$

(2) Case $\epsilon=-1$ :

$$
\begin{aligned}
(K, L, M, N)= & (1,6,2,3),(1,6,3,2),(2,15,5,6) \\
& (1,1,1,1),(1,2,1,2),(1,3,1,3),(1,2,2,1),(1,3,3,1),(2,9,9,2), \\
& (2,2,2,2) .
\end{aligned}
$$

Proof. (1) $\epsilon=+1$ :

Case $K=1$ : Then $P=K L+1=L+1=M N+1$.

[Subcase $M \leq N]$ We have $K^{2}+M^{2}=1+M^{2}=P$. Here we used that $K^{2}+M^{2} \equiv 0 \bmod P$ and $K^{2}+M^{2}<2 P$. By $M N+1=1+M^{2}$, we have $M=N$. Hence $(K, L, M, N)=(1,1,1,1)$ or $\left(1, m^{2}, m, m\right)$ with $m>1$.

[Subcase $N \leq M]$ We have $N^{2}<M N+1=P=L+1$. By $(L-1) P<L^{2}<L P$, we have $(L-1) P<L^{2}+N^{2}<(L+1) P$. Thus $L^{2}+N^{2} \equiv 0 \bmod P$ implies $L^{2}+N^{2}=L P$, 
thus $N^{2}=L$. By $M N+1=L+1=N^{2}+1$, we have $M=N$. We go back to the previous subcase.

Case $K=2$ : Then $P=K L+1=2 L+1=M N+1$.

[Subcase $M \leq N]$ We have $K^{2}+M^{2}=4+M^{2}=P$. By $M N+1=4+M^{2}$, we have $N=M+3 / M$. Hence $(K, L, M, N)=(2,2,1,4),(2,6,3,4)$. The former does not satisfy Condition $\mathrm{e} 3(3)$.

[Subcase $N \leq M]$ We have $N^{2}<M N+1=P=2 L+1$. By $(2 L-1) P<4 L^{2}<2 L P$, we have $(2 L-1) P<4\left(L^{2}+N^{2}\right)<(2 L+4) P$. Thus $L^{2}+N^{2} \equiv 0 \bmod P$ implies either $2\left(L^{2}+N^{2}\right)=L P$ or $2\left(L^{2}+N^{2}\right)=(L+1) P$. In the first case, $2 N^{2}=L$, thus $M N+1=$ $2 L+1=4 N^{2}+1$. We have $M=4 N$. Hence $(K, L, M, N)=\left(2,2 n^{2}, 4 n, n\right)$ with $n>1$, since $(2,2,4,1)$ does not satisfy Condition $\mathcal{C} 3(3)$. In the second case, $2 N^{2}=3 L+1$, thus $3(M N+1)=3(2 L+1)=4 N^{2}+1$. This has no integer solution.

(2) $\epsilon=-1$ : First, we study the cases where $K L=M N \leq 2$. It is easy to see that the solutions are $(K, L, M, N)=(1,1,1,1),(1,2,1,2),(1,2,2,1)$, where we care that $(K, L)=$ $(2,1)$ does not satisfy Condition $\mathcal{C} 3(3)$. Second, we study the cases where $(M, N)=(m, m)$ with $m>1$. The solution satisfying $K \leq 2$ is only $(K, L, M, N)=(2,2,2,2)$. The cases where $(M, N)$ is an exception (i.e., $(1,1),(1,2),(2,1)$ or $(m, m)$ with $m>1)$ are included in the first two cases above. Third, $(K, L)=(2,2)$ implies $(K, L, M, N)=(2,2,1,4),(2,2,4,1)$ or $(2,2,2,2)$, but the first two do not satisfy Condition $\mathrm{C} 3(3)$.

Now, we study the other cases: We assume that $(M, N)$ is not an exception (thus $M<N$ implies $M^{2}<M N-1=P, N<M$ implies $N^{2}<M N-1=P$ ) and that $L \geq 3$.

Case $K=1$ : Then $P=K L-1=L-1=M N-1$.

[Subcase $M<N]$ We have $K^{2}+M^{2}=1+M^{2}=P$. By $M N-1=1+M^{2}$, we have $N=M+2 / M$. Hence $(K, L, M, N)=(1,3,1,3),(1,6,2,3)$.

[Subcase $N>M$ ] We have $N^{2}<M N-1=P=L-1$. By $(L+1) P<L^{2}<(L+2) P$, we have $(L+1) P<L^{2}+N^{2}<(L+3) P$. Thus $L^{2}+N^{2} \equiv 0 \bmod P$ implies $L^{2}+N^{2}=(L+2) P$, thus $N^{2}=L-2$. If $L^{2}+N^{2}=(L+2) P$, by $M N-1=L-1=N^{2}+1$, we have $M=N+2 / N$. Hence $(K, L, M, N)=(1,3,3,1),(1,6,3,2)$.

Case $K=2$ : Then $P=K L-1=2 L-1=M N-1$.

[Subcase $M<N]$ We have $K^{2}+M^{2}=4+M^{2}=P$. By $M N-1=4+M^{2}$, we have $N=M+5 / M$. Hence $(K, L, M, N)=(2,3,1,6),(2,15,5,6)$.

[Subcase $N>M$ ] We have $N^{2}<M N-1=P=2 L-1$. By $(2 L+1) P<4 L^{2}<(2 L+2) P$, we have $(2 L+1) P<4\left(L^{2}+N^{2}\right)<(2 L+6) P$. Thus $L^{2}+N^{2} \equiv 0 \bmod P$ implies either $2\left(L^{2}+N^{2}\right)=(L+1) P$ or $2\left(L^{2}+N^{2}\right)=(L+2) P$. In the first case, $2 N^{2}=L-1$, thus $M N-1=$ $2 L-1=4 N^{2}+1$. We have $M=4 N+2 / N$. Hence $(K, L, M, N)=(2,3,6,1),(2,9,9,2)$. In the second case, $2 N^{2}=3 L-2$, thus $3(M N-1)=3(2 L-1)=4 N^{2}+1$. This has no integer solution.

Ignoring solutions that do not satisfy Condition C3 (3), we have the required list.

By $K L=M N$ in Condition $\mathrm{C} 3$ (1), if one of $K=M, K=N, L=M, L=N$ holds, then the pair is a trivial pair. We are mainly concerned with non-trivial pairs.

Suppose that $((K, L),(M, N))$ satisfies Condition $\mathcal{C} 3$ and also assume $K>2$. By (1) $K L=M N$ and coprimeness $(0)$ of $(K, L)$ and $(M, N)$, pairs $(K, M)$ and $(L, N)$ have common 
divisors. We set

$$
\begin{array}{llll}
\operatorname{gcd}(K, M)=d, & K=d K_{-}, & M=d M_{-}, & \operatorname{gcd}\left(K_{-}, M_{-}\right)=1 \\
\operatorname{gcd}(L, N)=D, & L=D L_{-}, & N=D N_{-}, & \operatorname{gcd}\left(L_{-}, N_{-}\right)=1
\end{array}
$$

By $K_{-} L_{-}=M_{-} N_{-}($from $K L=M N)$ and $\operatorname{gcd}\left(K_{-}, M_{-}\right)=\operatorname{gcd}\left(L_{-}, N_{-}\right)=1$, we have $K_{-}=N_{-}$and $L_{-}=M_{-}$. Thus we have

$$
(K, L, M, N)=\left(d K_{-}, D L_{-}, d L_{-}, D K_{-}\right), \quad \operatorname{gcd}\left(K_{-}, L_{-}\right)=1 .
$$

If $(K, L)$ is coprime, then $\operatorname{gcd}(d, D)=1$. But we do not assume it until Lemma 6.30.

Next, we use an identity

$$
\left(K^{2}+M^{2}\right)\left(L^{2}+N^{2}\right)=(K L-M N)^{2}+(K N+L M)^{2}=(K N+L M)^{2} .
$$

Here we used $K L=M N$. By (6.5), $(K N+L M) / P$ is an integer. We set $c=(K N+L M) / P$. Hence

$$
\begin{aligned}
& a P=K^{2}+M^{2}=d^{2}\left(\left(K_{-}\right)^{2}+\left(L_{-}\right)^{2}\right) \\
& b P=L^{2}+N^{2}=D^{2}\left(\left(K_{-}\right)^{2}+\left(L_{-}\right)^{2}\right) \\
& c P=K N+L M=d D\left(\left(K_{-}\right)^{2}+\left(L_{-}\right)^{2}\right) .
\end{aligned}
$$

We have

$$
\frac{c}{a}=\frac{b}{c}=\frac{D}{d}
$$

If $K_{-}=L_{-}(=1)$ or $d=D=1$, then the pair is a trivial pair. Thus we assume that $K_{-} \neq L_{-}$and $d<D$. By Condition $\mathrm{e} 3(3)$, for a non-trivial pair $((K, L),(M, N))$ with $K>2$, the order of $K, L, M, N$ is one of the followings. In accordance with the order of them, we classify pairs into two types:

$$
\text { (Type K) } K<N<M<L, \quad \text { (Type k) } K<M<N<L \text {. }
$$

Definition 6.26 (Reduction $\mathcal{R} 3$ ) For any pair $((K, L),(M, N))$ satisfying Condition $\mathrm{C} 3$ (1)-(3), we define a pair $\left(\left(K^{\prime}, L^{\prime}\right),\left(M^{\prime}, N^{\prime}\right)\right)$ as below: By using $x=\lceil L / N\rceil=\lceil M / K\rceil=$ $\left\lceil L_{-} / K_{-}\right\rceil \geq 2$,

$$
\left\{\begin{array} { l } 
{ K ^ { \prime } = x K - M } \\
{ L ^ { \prime } = N } \\
{ M ^ { \prime } = K } \\
{ N ^ { \prime } = x N - L }
\end{array} , \quad \text { whose inverse is } \left\{\begin{array}{l}
K=M^{\prime} \\
L=x L^{\prime}-N^{\prime} \\
M=x M^{\prime}-K^{\prime} \\
N=L^{\prime}
\end{array} .\right.\right.
$$

Note that $0 \leq K^{\prime}<K=M^{\prime}, 0 \leq N^{\prime}<N=L^{\prime}$. We call this operation from $((K, L),(M, N))$ to $\left(\left(K^{\prime}, L^{\prime}\right),\left(M^{\prime}, N^{\prime}\right)\right)$ reduction. By reduction, the minimum of the four integers decreases strictly: $K>K^{\prime} \geq 0$.

Lemma 6.27 In either case $\epsilon=+1$ or $\epsilon=-1$, the following holds:

$$
x=c, \quad \frac{x}{a}=\frac{b}{x}=\frac{D}{d} .
$$


Proof. First, we show

Claim. $L>x$.

Since $x=\lceil L / N\rceil$, we have $L>(x-1) N$ and

$$
L-x>(x-1) N-x=(x-1)(N-1)-1 .
$$

By $x \geq 2$ and $N>K>2$, we have the claim.

$$
\begin{aligned}
(x+1-c) P & =(x+1) P-(K N+L M) \\
& \geq(x+1)(K L-1)-(K N+L M) \\
& =(x K-M) L+K(L-N)-x-1 \\
& >(x K-M) L+K(L-N)-L-K \\
& =(x K-M-1) L+K(L-N-1) \geq 0, \\
(c-x+1) P & =K N+L M-(x-1) P \\
& \geq K N+L M-(x-1)(K L+1) \\
& =K N+\{M-(x-1) K\} L-x+1 \\
& >K N+\{M-(x-1) K\} L-L+1 \\
& =K N+\{M-(x-1) K-1\} L+1 \geq 0 .
\end{aligned}
$$

Thus we have $x-1<c<x+1$. We have $x=c$ as integers. The first equalities follows from (6.7).

Lemma 6.28 For any pair $((K, L),(M, N))$ satisfying Condition $\mathcal{C} 3$ (1)-(3) with $\epsilon$ and $\min \{K, L, M, N\}>2$, its reduction $\left(\left(K^{\prime}, L^{\prime}\right),\left(M^{\prime}, N^{\prime}\right)\right)$ without retaking satisfies Condition $\mathrm{C} 3$ (1)-(3) with $\epsilon$, with $P^{\prime}=x K N-P+2 \epsilon$. More precisely,

$$
\left(K^{\prime}\right)^{2}+\left(M^{\prime}\right)^{2}=a P^{\prime}, \quad\left(L^{\prime}\right)^{2}+\left(N^{\prime}\right)^{2}=b P^{\prime},
$$

where $a, b$ are integers defined by $K^{2}+M^{2}=a P, L^{2}+N^{2}=b P$, respectively.

Proof. (1)

$$
\begin{aligned}
K^{\prime} L^{\prime}+\epsilon & =(x K-M) N+\epsilon=x K N-M N+\epsilon=x K N-P+2 \epsilon \\
M^{\prime} N^{\prime}+\epsilon & =K(x N-L)+\epsilon=x K N-K L+\epsilon=x K N-P+2 \epsilon .
\end{aligned}
$$

(2) Since

$$
\begin{aligned}
x^{2} K^{2} & =x^{2}\left(d K_{-}\right)^{2}=x^{2} d^{2}\left(K_{-}\right)^{2}=a x d D\left(K_{-}\right)^{2}=a x K N \\
x K M & =x\left(d K_{-}\right)\left(d L_{-}\right)=x d^{2} K_{-} L_{-}=a d D K_{-} L_{-}=a K L \\
x^{2} N^{2} & =x^{2}\left(D K_{-}\right)^{2}=x^{2} D^{2}\left(K_{-}\right)^{2}=b x d D\left(K_{-}\right)^{2}=b x K N \\
x L N & =x\left(D L_{-}\right)\left(D K_{-}\right)=x D^{2} K_{-} L_{-}=b d D K_{-} L_{-}=b K L
\end{aligned}
$$


we have

$$
\begin{aligned}
\left(K^{\prime}\right)^{2}+\left(M^{\prime}\right)^{2} & =(x K-M)^{2}+K^{2}=K^{2}+M^{2}+x^{2} K^{2}-2 x K M \\
& =a P+a x K N-2 a K L=a P+a x K N-2 a(P-\epsilon) \\
& =a(x K N-P+2 \epsilon) \\
& =a P^{\prime} \\
\left(L^{\prime}\right)^{2}+\left(N^{\prime}\right)^{2} & =N^{2}+(x N-L)^{2}=L^{2}+N^{2}+x^{2} N^{2}-2 x L N \\
& =b P+b x K N-2 b K L=b P+b x K N-2 b(P-\epsilon) \\
& =b(x K N-P+2 \epsilon) \\
& =b P^{\prime} .
\end{aligned}
$$

(3) We have already shown $K^{\prime}<M^{\prime}$. It it easy in the case where the pair $((K, L),(M, N))$ is a trivial pair . For a non-trivial pair $((K, L),(M, N))$ of either Type $\mathrm{K}$ or Type $\mathrm{k}$, we have $K<N$ thus $M^{\prime}<L^{\prime}$. Since $K^{\prime} L^{\prime}=M^{\prime} N^{\prime}$ by (1), we have $K^{\prime}<N^{\prime}$. Thus $K^{\prime}$ is minimal among $\left\{K^{\prime}, L^{\prime}, M^{\prime}, N^{\prime}\right\}$. We have the lemma

We can summarize the argument above:

Lemma 6.29 Let $((K, L),(M, N))$ be a pair satisfying Condition $\mathcal{C} 3$ (1)-(3) with $\epsilon$ and $\min \{K, L, M, N\}>2$. We let $\left(\left(K^{\prime}, L^{\prime}\right),\left(M^{\prime}, N^{\prime}\right)\right)$ denote its reduction. Then either $(A)$ or (B) holds:

(A) It is one of Goals of Reduction $\mathcal{R} 3$, i.e., $\min \left\{K^{\prime}, L^{\prime}, M^{\prime}, N^{\prime}\right\} \leq 2$.

(B) Without retaking, $\left(\left(K^{\prime}, L^{\prime}\right),\left(M^{\prime}, N^{\prime}\right)\right)$ satisfies Condition $\mathrm{e} 3$ (1)-(3) with $\epsilon$. Furthermore, integers $a^{\prime}, b^{\prime}, x^{\prime}$ defined by

$$
x^{\prime}=\left\lceil M^{\prime} / K^{\prime}\right\rceil=\left\lceil N^{\prime} / L^{\prime}\right\rceil, \quad\left(K^{\prime}\right)^{2}+\left(M^{\prime}\right)^{2}=a^{\prime} P^{\prime}, \quad\left(L^{\prime}\right)^{2}+\left(N^{\prime}\right)^{2}=b^{\prime} P^{\prime}
$$

for the next reduction of $\left(\left(K^{\prime}, L^{\prime}\right),\left(M^{\prime}, N^{\prime}\right)\right)$ and integers $a, b, x$ defined by

$$
x=\lceil M / K\rceil=\lceil N / L\rceil, \quad K^{2}+M^{2}=a P, \quad L^{2}+N^{2}=b P
$$

of $((K, L),(M, N))$ satisfy

$$
x^{\prime}=x, \quad a^{\prime}=a, \quad b^{\prime}=b .
$$

Proof. Suppose that it is not the case (A), i.e., $\min \left\{K^{\prime}, L^{\prime}, M^{\prime}, N^{\prime}\right\}>2$. Almost all have been proved in Lemma 6.28. By Definition 6.26 and (6.5) (applied to $\left(\left(K^{\prime}, L^{\prime}\right),\left(M^{\prime}, N^{\prime}\right)\right)$ ) and the second half of Lemma 6.28, we have $a^{\prime}=a$ and $b^{\prime}=b$. The equality $x^{\prime}=x$ follows from $\left(x^{\prime}\right)^{2}=\left(c^{\prime}\right)^{2}=a^{\prime} b^{\prime}=a b=c^{2}=x^{2}$ by Lemma 6.27.

Next, we focus on coprimeness Condition $\mathcal{C} 3(0)$. Note that $K^{\prime}=0$ (and $N^{\prime}=0$ ) if and only if $M / K=L / N$ is an integer.

Lemma 6.30 Suppose that $((K, L),(M, N))$ is a pair satisfying Condition $\mathrm{C} 3$ (1)-(3) and $\min \{K, L, M, N\}>2$. Let $(K, L, M, N)=\left(d K_{-}, D L_{-}, d L_{-}, D K_{-}\right)$be the expression (6.6). If $((K, L),(M, N))$ satisfies coprimeness Condition $\mathrm{C} 3(0)$ in addition, then $K_{-}>1$. 
Proof. If $K_{-}=1$, then $(K, L, M, N)=(d, D x, d x, D)$ and $P=d D x+\epsilon$, where $x=\lceil M / K\rceil=$ $M / K$ is an integer with $x \geq 2$.

By $x P=c P=K N+L M=d D\left(x^{2}+1\right)$, we have

$$
\begin{aligned}
x(d D x+\epsilon) & =d D\left(x^{2}+1\right) \\
\epsilon x & =d D
\end{aligned}
$$

Thus $L=d D^{2}, \operatorname{gcd}(K, L)=d$. If $d>1$, then it contradicts Condition $\mathcal{C} 3(0)$, otherwise $K=d=1$ contradicts $\min \{K, L, M, N\}>2$. We have the lemma.

Lemma 6.31 Suppose that $((K, L),(M, N))$ is a pair satisfying Condition $\mathcal{C} 3$ (1)-(3) and $\min \{K, L, M, N\}>2$, and $\left(\left(K^{\prime}, L^{\prime}\right),\left(M^{\prime}, N^{\prime}\right)\right)$ is a reduction of $((K, L),(M, N))$, with $x=$ $\lceil M / K\rceil=\lceil L / N\rceil$. If $\operatorname{gcd}\left(K^{\prime}, L^{\prime}, x\right)>1$, then $\operatorname{gcd}(M, N, x)>1$. If $\operatorname{gcd}\left(M^{\prime}, N^{\prime}, x\right)>1$, then $\operatorname{gcd}(K, L, x)>1$.

Proof. It is easy to see by the inverse of Reduction $\mathcal{R} 3$ in Definition 6.26 .

Lemma 6.32 Let $((K, L),(M, N))$ be a pair satisfying Condition $\mathcal{C} 3$ (including (0)) with $\epsilon$ and $\min \{K, L, M, N\}>2$. If its reduction $\left(\left(K^{\prime}, L^{\prime}\right),\left(M^{\prime}, N^{\prime}\right)\right)$ satisfies $\min \left\{K^{\prime}, L^{\prime}, M^{\prime}, N^{\prime}\right\} \leq$ 2 , then either $(+)$ or $(-)$ holds:

(+) Case $\epsilon=+1$

$((K, L),(M, N))$ is a trivial pair.

(-) Case $\epsilon=-1$

$((K, L),(M, N))$ is a trivial pair, or

(i) $\left(\left(K^{\prime}, L^{\prime}\right),\left(M^{\prime}, N^{\prime}\right)\right)=((2,15),(5,6))$ and $(a, b)=(1,9), x=3$.

(ii) $\left(\left(K^{\prime}, L^{\prime}\right),\left(M^{\prime}, N^{\prime}\right)\right)=((1,6),(3,2))$ and $(a, b)=(2,8), x=4$.

Proof. Suppose that $((K, L),(M, N))$ is not a trivial pair. The reduction $\left(\left(K^{\prime}, L^{\prime}\right),\left(M^{\prime}, N^{\prime}\right)\right)$ satisfies Condition C3 (1)-(3) by Lemma 6.28, and the equalities $a^{\prime}=a, b^{\prime}=b$ hold.

Claim 1. $K^{\prime} \neq 0$ and $N^{\prime} \neq 0$.

Since $K^{\prime}=0$ means that $M / K(=L / N)$ is an integer, and $K_{-}=1$. It contradicts the assumption that $((K, L),(M, N))$ satisfies Condition $\mathcal{C} 3(0)$ by Lemma 6.30 .

Claim 2. In the list in Lemma 6.32 , only in the case $\epsilon=-1$, only three pairs

$$
(1,6,3,2),(1,6,2,3),(2,15,5,6)
$$

are possible as $\left(\left(K^{\prime}, L^{\prime}\right),\left(M^{\prime}, N^{\prime}\right)\right)$, and the others contradict coprimeness Condition $\mathrm{e} 3(0)$ or non-triviality on $((K, L),(M, N))$.

This is verified one by one, by using inverse of Reduction $\mathcal{R} 3$ and Lemma 6.31 .

We study the integers $a^{\prime}, b^{\prime}$ and $x^{\prime}$ for three cases.

Case 0. $\left(K^{\prime}, L^{\prime}, M^{\prime}, N^{\prime}\right)=(1,6,2,3)$ with $\left(a^{\prime}, b^{\prime}\right)=(1,9), x^{\prime}=3$.

Case 1. $\left(K^{\prime}, L^{\prime}, M^{\prime}, N^{\prime}\right)=(1,6,3,2)$ with $\left(a^{\prime}, b^{\prime}\right)=(2,8), x^{\prime}=4$.

Case 2. $\left(K^{\prime}, L^{\prime}, M^{\prime}, N^{\prime}\right)=(2,15,5,6)$ with $\left(a^{\prime}, b^{\prime}\right)=(1,9), x^{\prime}=3$.

Case 0 contradicts the assumption, since $\left(\left(K^{\prime}, L^{\prime}\right),\left(M^{\prime}, N^{\prime}\right)\right)=((1,6),(2,3))$ only if $((K, L),(M, N))=$ $((2,15),(5,6))$, which does not satisfy $\min \{K, L, M, N\}>2$. We have the lemma. 
Theorem 6.33 Any pair $((K, L),(M, N))$ that satisfies Condition $\mathcal{C} 3$ is in the sequence $[K L M N]$ or $[k l m n]$ (see Definition 1.15). In other words, $((K, L),(M, N))=\left(\left(K_{i}, L_{i}\right),\left(M_{i}, N_{i}\right)\right)$ in the sequence $[K L M N]$, or $((K, L),(M, N))=\left(\left(k_{i}, l_{i}\right),\left(m_{i}, n_{i}\right)\right)$ in the sequence $[k l m n]$, for a positive integer $i$.

Proof. The method of the proof is similar to the proof of Theorem 6.23 in the cases [STUV] and $[$ stuv].

By Lemma 6.29, we can iterate reductions on $((K, L),(M, N))$, until Case (A) occurs. The goal is $((2,15),(5,6))\left(=\left(\left(K_{2}, L_{2}\right),\left(M_{2}, N_{2}\right)\right)\right.$ with $x=3$, or $((1,6),(3,2))\left(=\left(\left(k_{1}, l_{1}\right),\left(m_{1}, n_{1}\right)\right)\right.$ with $x=4$. Since the inverse of Reduction $\mathcal{R} 3$ with $x=3$ or 4 agrees with the construction of the sequence $[K L M N]$ or $[k l m n]$, the pair $((K, L),(M, N))$ is in $[K L M N]$, or in $[k l m n]$, respectively.

Acknowledgement. The authors would like to express sincere gratitude to Professor Masakazu Teragaito and Professor Toshio Saito for various communication on lens space surgeries and for giving the authors valuable advice. The authors thank Professor Hirofumi Sasahira for his interest in our results from another view point. The authors would like to express sincere gratitude to Professor Masaaki Ue. This research started when the first author talked on Problem 1.1 for our first example in Ue's seminor. The authors thank anonymous referee for careful reading of the manuscript.

\section{References}

[A] S Akbulut, Dolgachev surface, arXiv:math.GT/08051524.

[Boy] S Boyer, Simply-connected 4-manifolds with a given boundary, Trans. Amer. Math. Soc. 298 (1986), no. 1, 331-357.

[Bon] F Bonahon, Diffeotopies des espaces lenticulaires, (French) [Diffeotopies of lens spaces], Topology 22 (1983), no. 3, 305-314.

[GS] R Gompf and A Stipsicz, 4-manifolds and Kirby calculus, Grad. Studies in Math. 20 A. M. S. (1999).

[HR] C Hodgson and J H Rubinstein, Involutions and isotopies of lens spaces, Knot theory and manifolds (Vancouver, B.C., 1983), 60-96, Lecture Notes in Math., 1144, Springer, Berlin, (1985).

[K] R Kirby, A calculus for framed links in $S^{3}$, Invent. Math. 45 (1978), 35-56.

[K2] R Kirby, The topology of four manifolds, Lecture Notes in Mathematics, 1374. Springer-Verlag, Berlin (1989).

[Mo] L Moser, Elementary surgery along a torus knot, Pacific J. Math. 38 (1971), 737-745.

[R] D Rolfsen, Knots and links, Publish or Perish, Berkeley (1976).

[ST] T Saito and M Teragaito, Knots yielding homeomorphic lens spaces by Dehn surgery, Pacific J. Math. 244 no.1 (2010), 169-192.

[Sa] H Sasahira, Instanton Floer homology for lens spaces, to appear in Math. Z., (arXiv:math.GT1009.0331v2).

[U] M Ue, On the intersection forms of spin 4-manifolds bounded by spherical 3-manifolds, Algebr. Geom. Topol. 1 (2001), 549-578. 
[Y1] Y Yamada, Berge's knots in the fiber surfaces of genus one, lens spaces and framed links, J. Knot Theory Ramifications 14 (2005), no.2, 177-188.

[Y2] Y Yamada, A family of knots yielding graph manifolds by Dehn surgery, Michigan Math. J. 53(3) (2005), 683-690.

\section{Motoo Tange}

Research Institute for Mathematical Sciences, Kyoto University

Kitashirakawa Oiwake Sakyo-ku Kyoto-shi 606-8502, JAPAN

tange $=A T=$ kurims $\cdot$ kyoto $-u \cdot a c \cdot$.jp

Current address: Institute of Mathematics, University of Tsukuba,

1-1-1 Tennodai, Tsukuba, Ibaraki 305-8571, Japan

tange $=A T=$ math $\cdot$ tsukuba $\cdot$ ac $\cdot$ jp

Yuichi Yamada

Dept. of Mathematics, The Univ. of Electro-Communications

1-5-1,Chofugaoka, Chofu, Tokyo, 182-8585, JAPAN

yyyamada $=A T=$ sugaku $\cdot e-$ one $\cdot u e c \cdot a c \cdot j p$ 

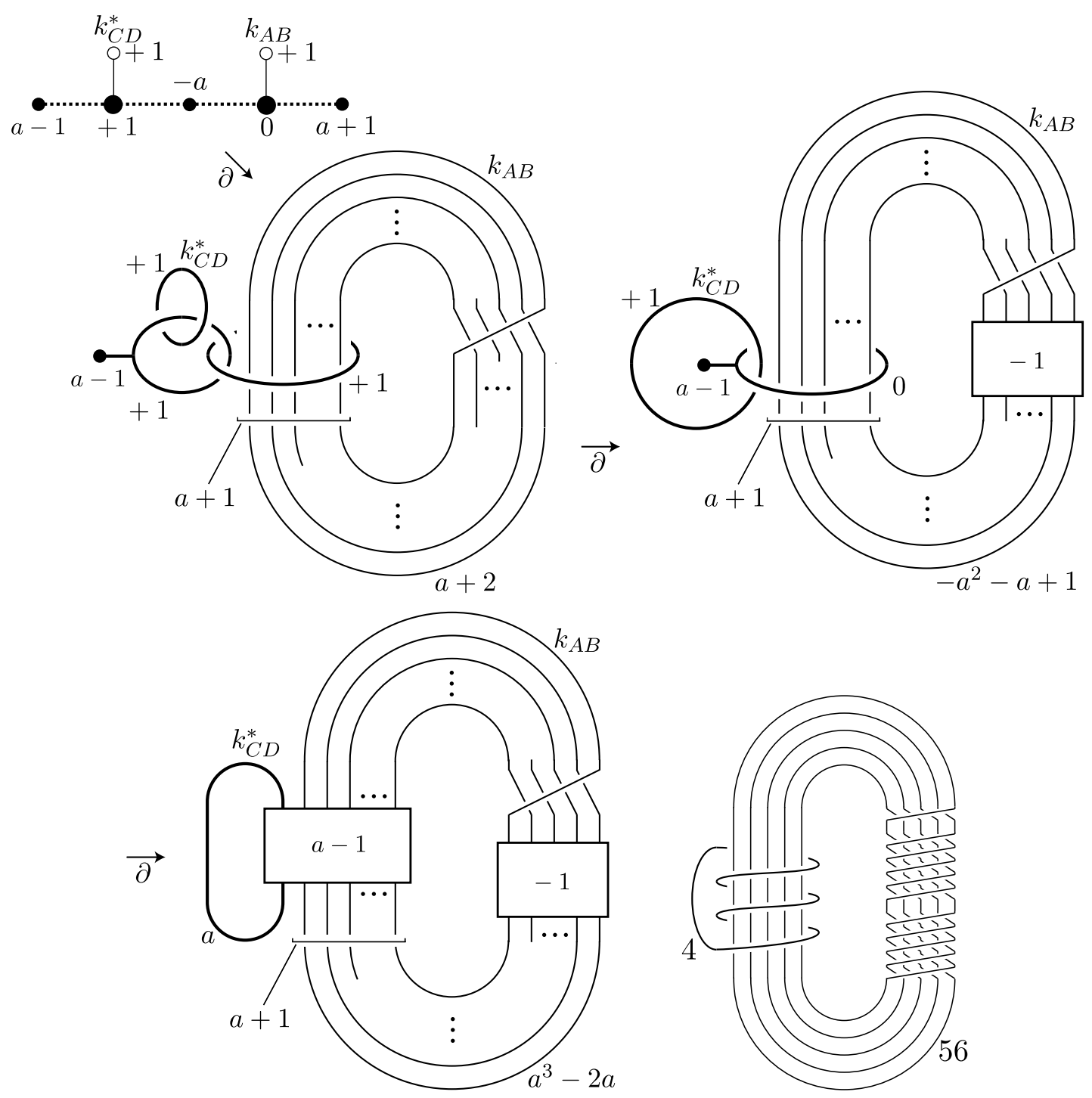

Figure 25: Kirby Diagram of $X_{A B} \cup\left(-X_{C D}\right)(i=2), \quad($ ex. $a=4)$ 


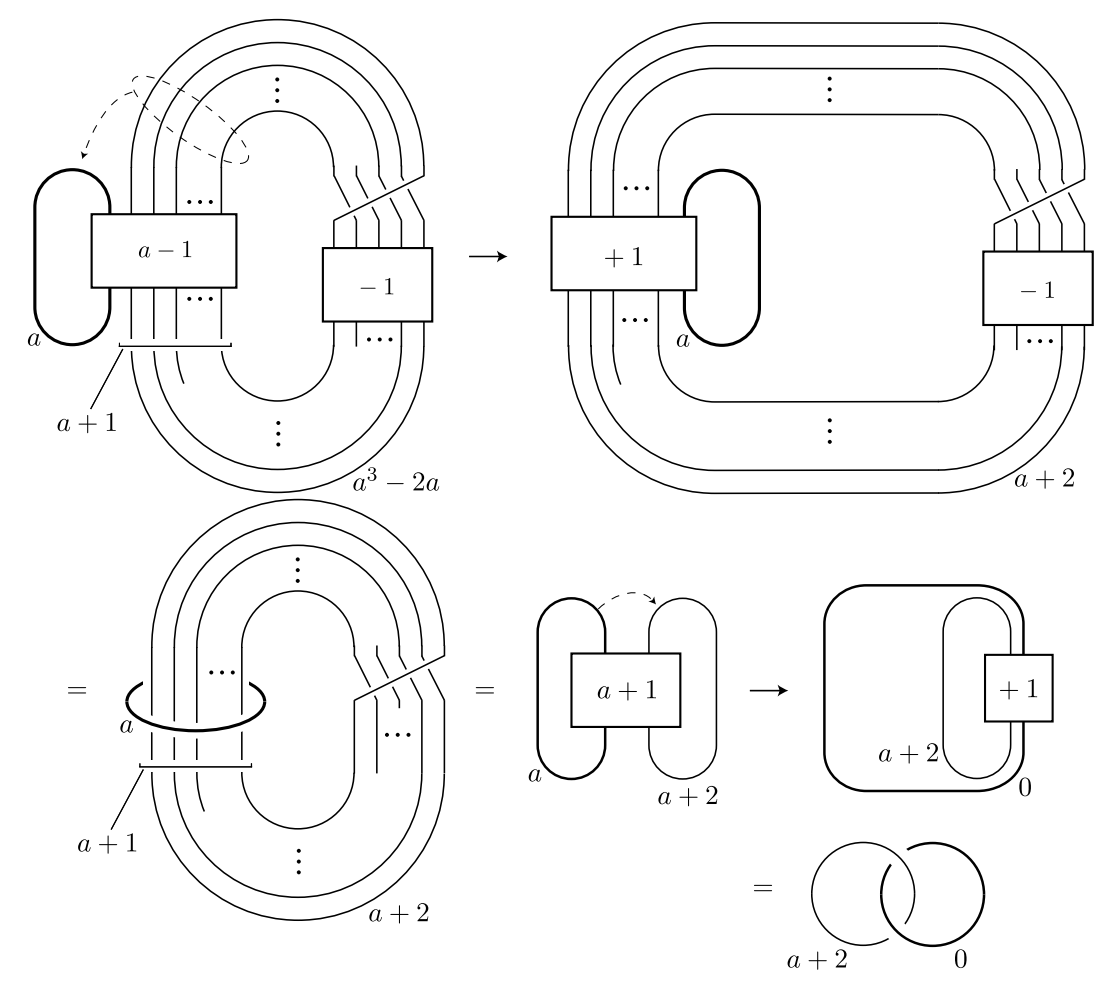

Figure 26: Kirby calculus to the required manifold $(i=2)$ 